\title{
COMPUTATIONAL MODELING TOWARDS ACCELERATING ACCIDENT TOLERANT FUEL CONCEPTS AND DETERMINING IN-PILE FUEL BEHAVIOR
}

by

Ember Sikorski

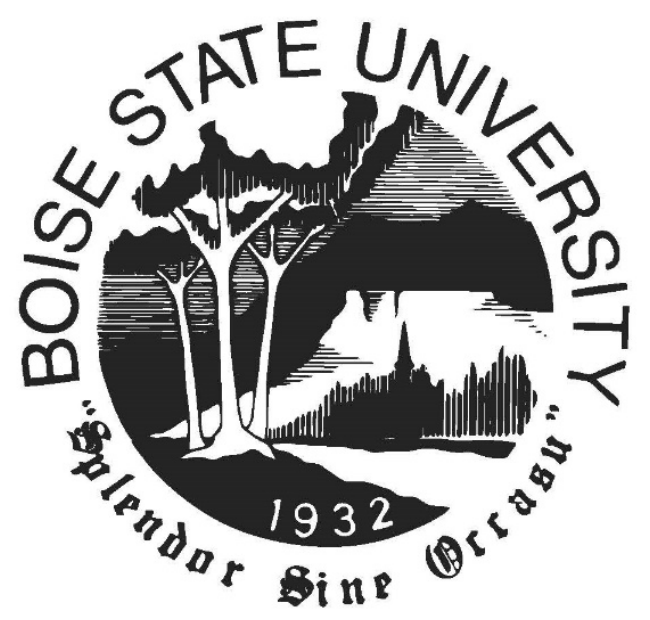

A dissertation

submitted in partial fulfillment

of the requirements for the degree of

Doctor of Philosophy in Materials Science and Engineering

Boise State University

August 2021 
(C) 2021

Ember Sikorski

ALL RIGHTS RESERVED 


\title{
BOISE STATE UNIVERSITY GRADUATE COLLEGE
}

\section{DEFENSE COMMITTEE AND FINAL READING APPROVALS}

\author{
of the dissertation submitted by
}

\section{Ember Sikorski}

Dissertation Title: Computational Modeling Towards Accelerating Accident Tolerant Fuel Concepts and Determining In-Pile Fuel Behavior

Date of Final Oral Examination:

25 June 2021

The following individuals read and discussed the dissertation submitted by student Ember Sikorski, and they evaluated their presentation and response to questions during the final oral examination. They found that the student passed the final oral examination.

Lan (Samantha) Li, Ph.D. Chair, Supervisory Committee

Brian J. Jaques, Ph.D. Member, Supervisory Committee

Eric Jankowski, Ph.D. Member, Supervisory Committee

Larry K. Aagessen, Ph.D. Member, Supervisory Committee

Richard S. Skifton, Ph.D. Member, Supervisory Committee

The final reading approval of the dissertation was granted by Lan (Samantha) Li, Ph.D., Chair of the Supervisory Committee. The dissertation was approved by the Graduate College. 


\section{DEDICATION}

To my parents, Michael and Brenda, for exemplifying resilience and standing up for what you believe in. To Kevin for his never-ending support. 


\section{ACKNOWLEDGMENTS}

I owe gratitude to a large number of people, and the following will surely not be exhaustive.

Thank you to my advisor, Dr. Lan Li. I cannot express enough how grateful I am for the opportunities you have sought out for me, the time you dedicate to your students, and the mentorship you have provided in all areas required to be a successful scientist.

Thank you to my committee members Brian Jaques, Eric Jankowski, Richard Skifton, and Larry Aagesen. You have all helped me either through classes that have been essential to my research, collaboration, advice, introducing me to BSU, and/or mentorship during my time at INL.

To the other members of the Materials Theory and Modeling Group: Matthew Lawson, Izaak Williamson, Austin Biaggne, and German Barcenas. We have learned together and fought off more errors than I can count.

To Jennifer Watkins and Scott Riley for making our collaborations a rewarding experience.

To my friends Rebecca Hernández, Thiago da Silva, Jenny Fothergill, Cait Brunkhorst, Matty Jones, Bryce Eveland, Julian Antolín Camarena, Esther Aivesue Rodríguez Hernández, Rebecca Miller, Kaelee Novich, and Kelly McCary who have helped me to survive and enjoy the last five years.

To Kenneth Holzman, for helping me discover my strengths and passion for science and public speaking. 
To Ben Nickell and the rest of the INL HPC staff for their extensive help over the years. To Jamie Hayward, Taylor Hartung, and the rest of the MSMSE staff who keep everything running smoothly and have gotten me to all of the conferences I have attended. To Jessica Economy, for all the coordination you do for the department, your ever appreciated third-person perspective, and consistent answers to a barrage of questions. To Wayne Cochrane and the rest of the graduate college staff who make 3MT possible, for not letting me give up, and providing an amazing opportunity.

Thank you to Choco, Coffee, Tea, and Chai who have been the best company I could ask for in the middle of a pandemic.

Thank you to the Center for Advanced Energy Studies for supporting this effort, providing access, and enabling collaboration that otherwise may have been unavailable.

This research made use of the resources of the High Performance Computing Center at Idaho National Laboratory, which is supported by the Office of Nuclear Energy of the U.S. Department of Energy and the Nuclear Science User Facilities under Contract No.DE-AC07-05ID14517. Further computational resources were provided by the R2 cluster (DOI: 10.18122/B2S41H) provided by Boise State University's Research Computing Department. This work was supported in part through the Department of Energy Advanced Sensors and Instrumentation program under DOE Idaho Operations Office Contract DE-AC07-05ID14517. The views and opinions of authors expressed herein do not necessarily state or reflect those of the U.S. Government or any agency thereof. 


\begin{abstract}
To mitigate global warming, we need to develop carbon-free ways to generate power. Nuclear energy currently generates more carbon-free power in the United States than all other sources combined at 55\%. To make nuclear as viable a power source as possible, we need to maximize power density and safety. Both of these can be improved with Accident Tolerant Fuel (ATF) materials. Uranium nitride (UN), a candidate ATF material, offers high fuel economy due to its uranium density and improved safety margins from thermal properties. However, its instability in the presence of water, a reactor coolant, must be addressed. This dissertation employs Density Functional Theorybased methods to investigate the atomistic and electronic mechanisms in UN corrosion initiation. To ensure accuracy in future UN models, the effects of magnetic treatments on UN surface stability and corrosion properties are also determined.

The performance of advanced nuclear materials must be tested in research reactors before they can be implemented in power reactors. To get real-time temperature data from these tests, sensors are required that can survive the high temperatures and irradiation. To meet these needs, Idaho National Laboratory has been developing High Temperature Irradiation Resistant Thermocouples (HTIR-TCs). Towards increasing temperature resolution and in-pile lifetime, an ab initio method has been developed to predict HTIR-TC performance. The method considers the effects of composition and temperature on performance and has been validated against experiment. To predict the interaction of HTIR-TCs with research reactor coolant, corrosion and oxidation
\end{abstract}


mechanisms have been investigated. By examining the diffusion behaviors of water and oxygen, recommendations are made for which thermoelement materials may be the most resistant to corrosion and/or oxidation. 


\section{TABLE OF CONTENTS}

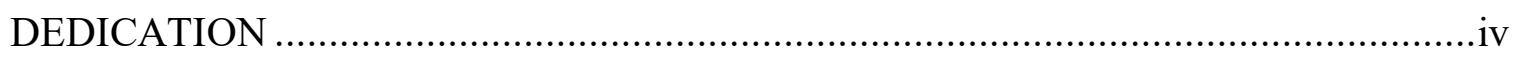

ACKNOWLEDGMENTS ............................................................................

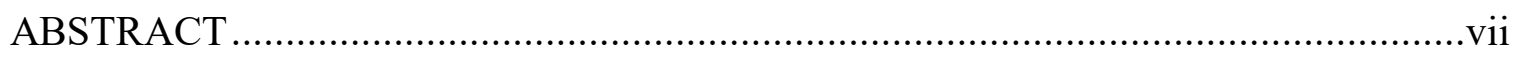

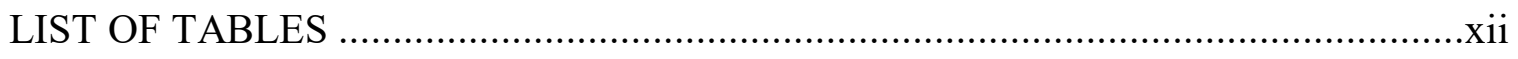

LIST OF FIGURES ............................................................................. xiii

LIST OF ABBREVIATIONS ...................................................................

CHAPTER ONE: INTRODUCTION .............................................................. 1

1.1 Goal of the Dissertation...................................................................... 1

1.2 Background, Motivation, and Objectives ........................................... 2

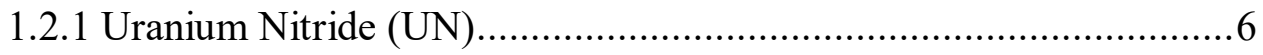

1.2.2 High Temperature Irradiation Resistant Thermocouples (HTIR-TCs)

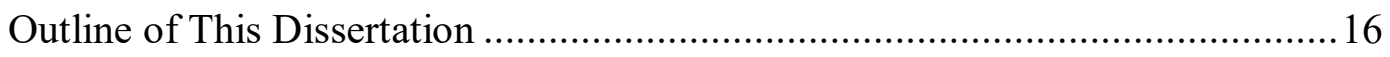

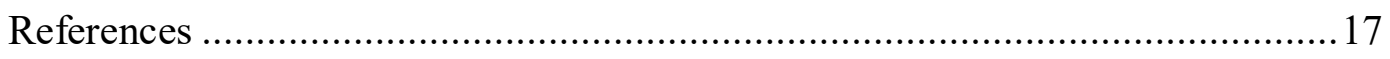

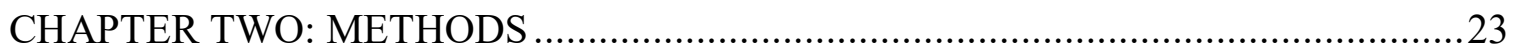

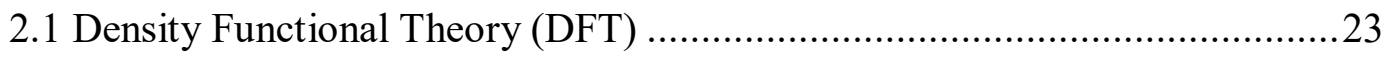

2.2 Ab initio Molecular Dynamics (AIMD) ............................................ 28

2.3 Methods Implemented in This Dissertation............................................29

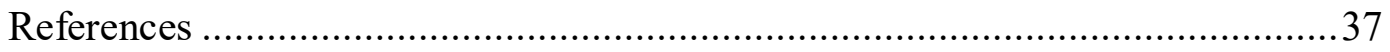




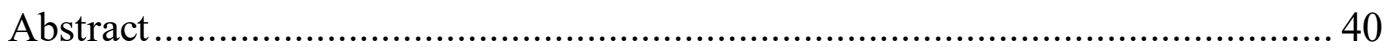

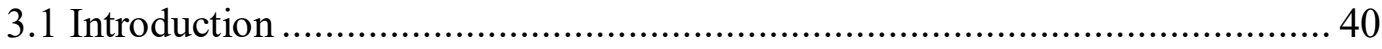

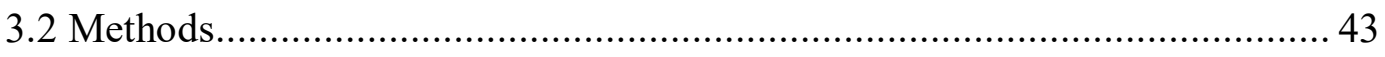

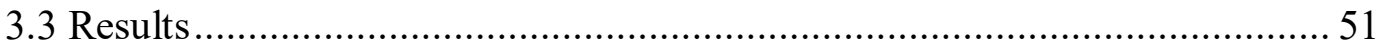

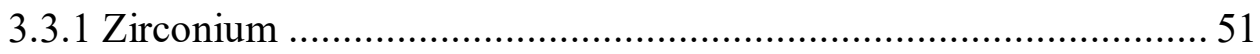

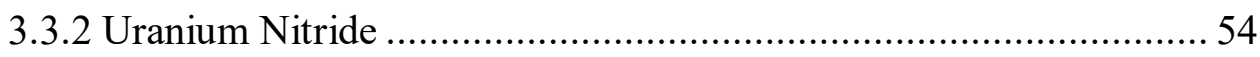

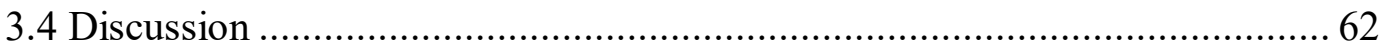

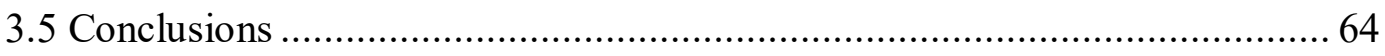

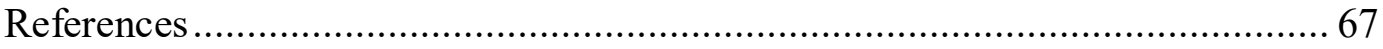

CHAPTER FOUR: MAGNETIC PROPERTIES OF THE URANIUM NITRIDE

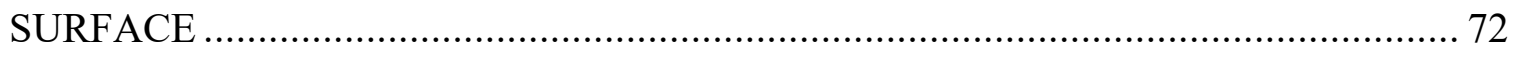

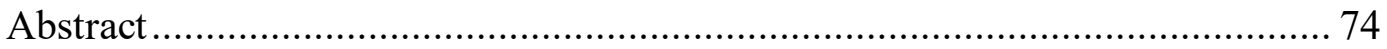

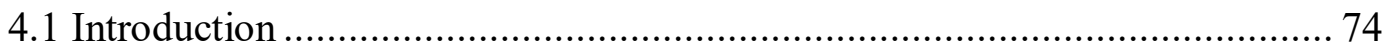

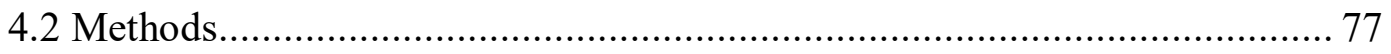

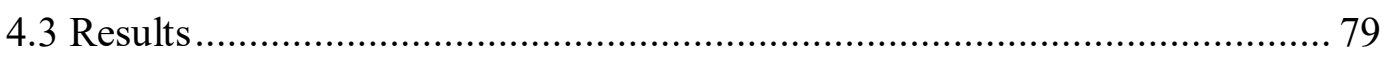

4.3.1 Magnetic Structure ..................................................................... 79

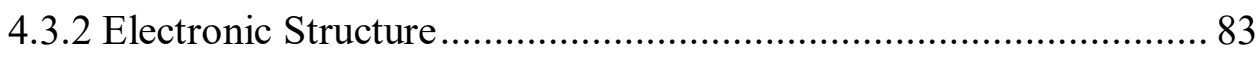

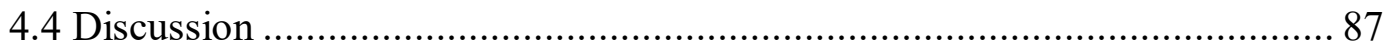

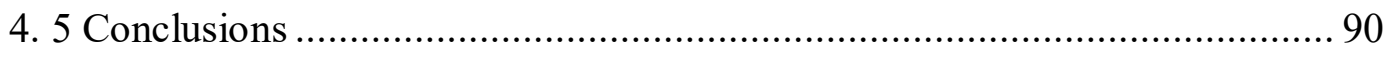

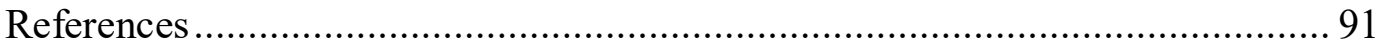

CHAPTER FIVE: COMBINED EXPERIMENT AND FIRST-PRINCIPLES STUDY OF IN-PILE TEMPERATURE SENSOR MATERIALS ………………….................... 93

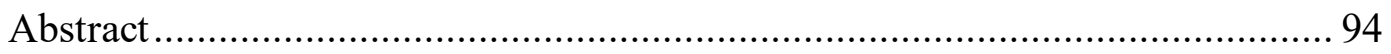




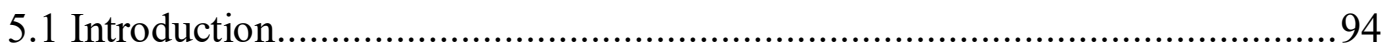

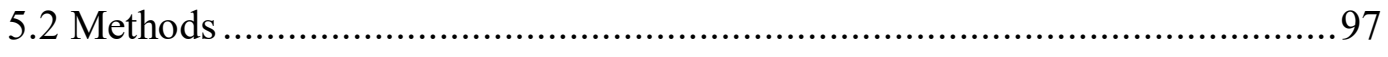

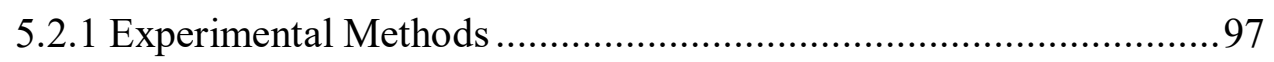

5.2.2 Computational Methods ................................................... 97

5.3.1 Experimental Voltage Measurement ..................................... 100

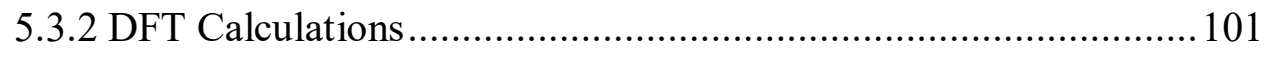

5.3.3 Ab-Initio Molecular Dynamic Simulations............................. 105

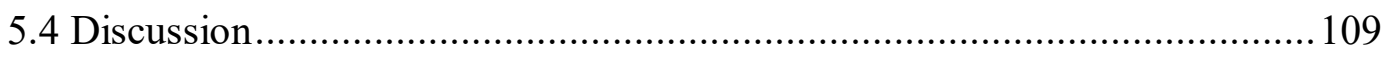

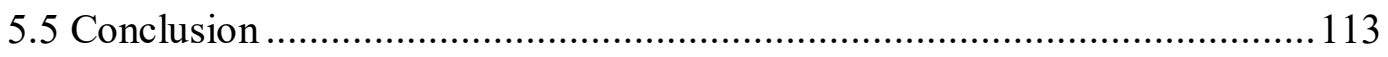

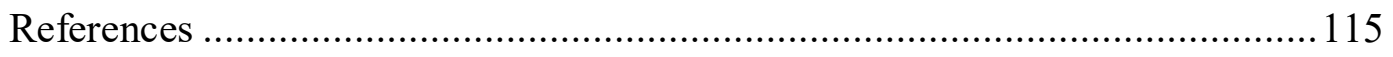

CHAPTER SIX: OXIDATION AND CORROSION OF IN-PILE TEMPERATURE

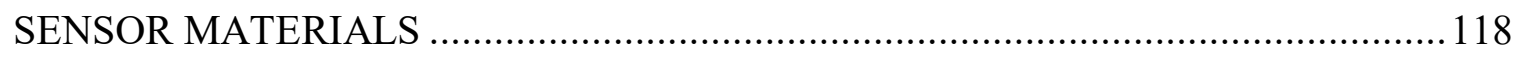

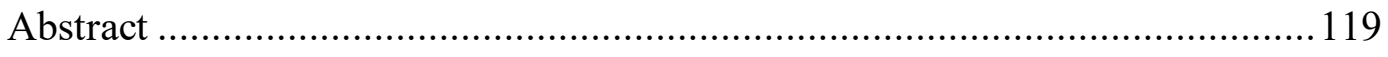

6.1 Introduction.............................................................................. 119

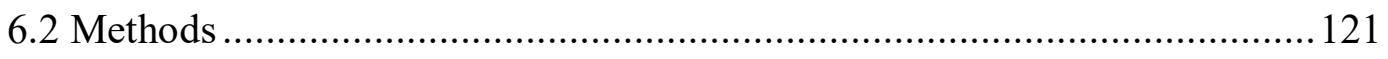

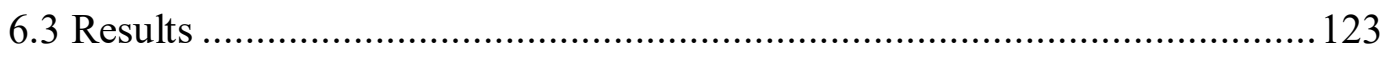

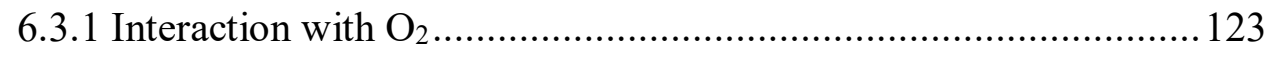

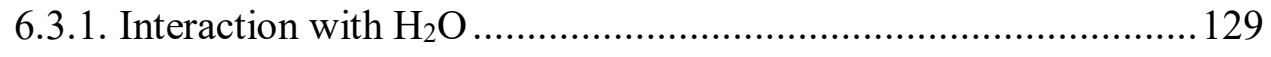

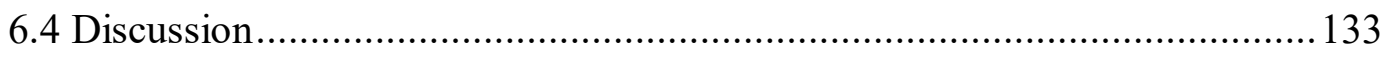

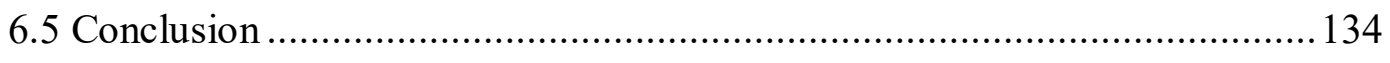

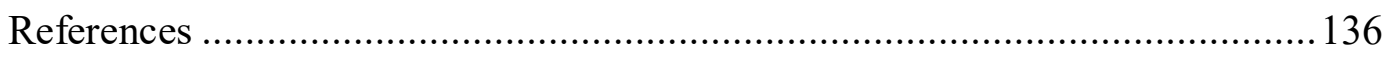

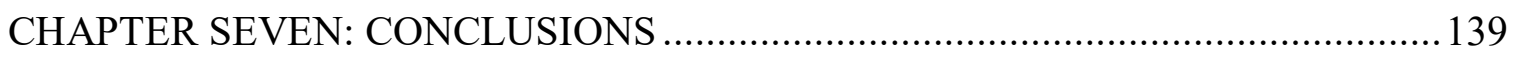




\section{LIST OF TABLES}

Table 3.1 Calculated binding energies for O at U-top and N-top sites on 4-, 6- and 8monolayer slabs.

Table 3.2 Calculated UN lattice constant in comparison with experimental and theoretical data.

Table 3.3 Calculated UN bulk modulus (GPa) in comparison with experimental and theoretical data.

Table 3.4 Surface energies from this work, Bo et al., and Bocharov et al. for a 5monolayer slab in terms of eV/ $\AA^{2}$. Values in parenthesis are given in $\mathrm{J} / \mathrm{m}^{2}$.

Table 4.1 Magnetic moments $(\boldsymbol{\mu} \boldsymbol{B})$ of $U$ atoms averaged over each (100) layer after

U-ramping to $\mathrm{U}_{\text {eff }}=2.0 \mathrm{eV}$...... 82

Table 4.2 Bond lengths, bond angles, vertical displacement of the $U$ atom bonded to $\mathrm{OH}$ after geometry optimization, and adsorption energy for FM, AFM spin-up terminated, AFM spin-down terminated, and NM magnetic structures. Two O-U-N bond angles are reported for each structure: one considering the neighboring $\mathrm{N}$ atom pictured to the right and one considering the $\mathrm{N}$ atom into the page in Figure 4.3.

Table 5.1 Incorporation energies of $\mathrm{O}$ in $\mathrm{Nb}$ and $\mathrm{Mo}$ when substituting a metal atom far from, at the 1 st nearest neighbor, or at the 2 nd nearest neighbor site relative to the HTIR additive $\mathrm{P}$ or La, respectively.

Table 6.1 Diffusion coefficient pre-factor and activation energy for $\mathrm{O} 2$ at each surface.

Table 6.2 Diffusion coefficient pre-factors and activation energies of $\mathrm{O}$ and $\mathrm{H}$ for each surface exposed to $\mathrm{H}_{2} \mathrm{O}$. 


\section{LIST OF FIGURES}

Figure 1.1 An example of materials models that can be used in multi-scale mechanistic modeling, with respect to length and time.

Figure 1.2 Schematic of a HTIR-TC consisting of two thermoelements: HTIR-Mo and HTIR-Nb which act as the positive and negative thermoelement, respectively. Each thermoelement has its own respective Seebeck coefficient, $\boldsymbol{\sigma}$. One end of the thermocouple is placed into a reactor to measure a temperature, $T_{l}$, while the other end is held at a constant temperature, $T_{0}$, outside of the reactor. Due to the Seebeck effect, the thermocouple produces a voltage, $E$, proportional to temperature difference, $T_{1}-T_{0}$.

Figure 1.3 Cross sections of (a) the traditional thermocouple build and (b) the coaxial thermocouple build including two thermoelements: $\boldsymbol{\sigma} \mathbf{1}$ and $\boldsymbol{\sigma} \mathbf{2}$.

Figure 2.1 Methods used in this dissertation. Methods marked with blue stars and orange diamonds were used for the UN corrosion and HTIR-TC projects,

respectively. 30

Figure 2.2 An example of Local Density of States (LDOS) for an OH adsorbed to a UN surface. The Fermi energy has been shifted to $0 \mathrm{eV}$ by convention...31

Figure 2.3 Partial Charge Density (PCD) from -2 eV to the Fermi energy for an $\mathrm{OH}$ adsorbed to a UN surface. The scale has been narrowed to $25 \%$ of the total electronic states to improve the visibility of bonds. Contour lines indicate areas with the same energy. Blue and red indicate many and no electronic states, respectively. Some $\mathrm{U}$ and $\mathrm{N}$ atoms have been numbered for reference in the text.

Figure 2.4 Example of Electron Localization Function (ELF) for an $\mathrm{OH}$ adsorbed to a UN surface. Blue and red indicate high and low probability of finding a localized electron, respectively. Contour lines indicate areas with the same energy.

Figure 2.5 Example of using the Radial Distribution Function for the HTIR-Mo system as it is heated from 20 to $1600{ }^{\circ} \mathrm{C}$. The peaks at $20^{\circ} \mathrm{C}$ are numbered for discussion in the text. 
Figure 2.6 Example of using Nudged Elastic Band (NEB) to find the transition energy of (a) an $\mathrm{O}$ atom moving from an adsorbed hollow site to an interstitial site in Mo. (b) Energies for the initial and final position of the $\mathrm{O}$ atom as well as 6 intermediary images......................................... 35

Figure 2.7 O diffusion coefficients for HTIR thermoelements exposed to $\mathrm{O}_{2}$. ........36

Figure 3.1 Comparison of the Partial Charge Density (PCD) (a-b) and Electron Localization Function (ELF) (c-d) for O adsorption on an 4-monolayer slab (left) and 8-monolayer slab (right). The $\mathrm{O}$ was placed on top of different $U$ atoms between the 4- and 8-monolayer slab, resulting in a lateral shift of the charge maps for (a) and (c). Contour lines indicate areas with the same energy. In the ELF, 0 represents low probability of finding an electron and 1 represents high probability of finding an electron. The PCD shows the density of states from $-2 \mathrm{eV}$ to the Fermi level mapped onto the structure. The PCD map has been narrowed to $30 \%$ of the total states to better visualize the bonds.

Figure 3.2 Initial configurations: $\mathrm{UN}$ and $\mathrm{Zr}$ surfaces with $\mathrm{OH}+\mathrm{H}(\mathrm{a}-\mathrm{c})$ and $\mathrm{OH}+\mathrm{H}+\mathrm{O}$ (d-f). Top-down view of adsorption sites on UN (100) (g). U, N, Zr, O, and $\mathrm{H}$ are represented by blue, purple, teal, red, and gray, respectively... 50

Figure 3.3 Top-down view comparison between initial configurations of UN surfaces (a-d) and relaxed configurations of $\mathrm{UN}$ surfaces (e-h). $\mathrm{U}, \mathrm{N}, \mathrm{O}$, and $\mathrm{H}$ are represented by blue, purple, red, and gray, respectively. 50

Figure 3.4 Local density of states of (a) $\mathrm{Zr}$ (1000) surface with $\mathrm{OH}+\mathrm{H}$ and (b) with $\mathrm{OH}+\mathrm{H}+\mathrm{O}$.

Figure 3.5 Relaxed configurations: $\mathrm{UN}$ and $\mathrm{Zr}$ surfaces with $\mathrm{OH}+\mathrm{H}(\mathrm{a}-\mathrm{c})$ and $\mathrm{OH}+\mathrm{H}+\mathrm{O}(\mathrm{d}-\mathrm{f}) . \mathrm{U}, \mathrm{N}, \mathrm{Zr}, \mathrm{O}$, and $\mathrm{H}$ are represented by blue, purple, teal, red, and gray, respectively.

Figure 3.6 Electron Localization Function (ELF) of (a) the pure $\operatorname{Zr}$ (1000) surface, (b) with $\mathrm{OH}+\mathrm{H}$, and (c) with $\mathrm{OH}+\mathrm{H}+\mathrm{O}$. Blue and red indicate high and low probability of finding a localized electron, respectively. Contour lines indicate areas with the same energy............................................ 54

Figure 3.7 UN (100) after $\mathrm{H}_{2} \mathrm{O}$ and $\mathrm{O}_{2}$ adsorption: $\mathrm{U}, \mathrm{N}, \mathrm{O}$, and $\mathrm{H}$ are represented by blue, purple, red, and gray, respectively.

Figure 3.8 Local density of states of (a) UN (100) with $\mathrm{OH}+\mathrm{H}$, (b) UN (100) with $\mathrm{OH}+\mathrm{H}+\mathrm{O}$, (c) $\mathrm{UN}(110) \mathrm{OH}+\mathrm{H}$, and (d) $\mathrm{UN}$ (110) with $\mathrm{OH}+\mathrm{H}+\mathrm{O}$. Individual contributions from $\mathrm{U}$ and $\mathrm{N}$ are shown in (a), while $\mathrm{U}$ and $\mathrm{N}$ contributions are combined in (b-d) to indicate bonding between the adsorbates and the surface in general. 
Figure 3.9 Partial charge density of the UN (100) surface without dissociated water, with dissociated water, and with dissociated water and added oxygen. The scale has been narrowed to $30 \%$ of the total electronic states and contour lines added to improve the visibility of bonds. Blue and red indicate many and no electronic states, respectively.

Figure 3.10 Partial charge density of the UN (110) surface without dissociated water, with dissociated water, and with dissociated water and added oxygen.....61

Figure 3.11 Electron localization function of the UN (110) surface without dissociated water, with dissociated water, and with dissociated water and added oxygen. .62

Figure 4.1 Initial magnetic structures applied during $U$-ramp to $\mathrm{U}_{\mathrm{eff}}=2.0 \mathrm{eV} \ldots \ldots \ldots . .79$

Figure 4.2 Relative energy (eV) of the magnetic structures with respect to $U_{\text {eff }}$ during the U-ramp to $2.0 \mathrm{eV}$. Energies are given with respect to the FM total energy at each respective $U_{\text {eff }}$ value. Note that the AFM values correspond to the structure that was initialized as type 1 AFM, corresponding to bulk $\mathrm{UN}$, before the U-ramp but transitioned to a new magnetic structure as shown in Table 4.1 . .81

Figure 4.3 Atomic structures after $\mathrm{OH}$ adsorption for FM, AFM spin-up terminated, AFM spin-down terminated, and NM structures. Blue, purple, red, and white atoms represent $\mathrm{U}, \mathrm{N}, \mathrm{O}$, and $\mathrm{H}$, respectively. .84

Figure 4.4 Local Densities of States for OH relaxed on (a) FM, (b) AFM, and (c) NM slabs. The Fermi energy is shifted to $0 \mathrm{eV}$.

Figure 4.5 Partial Charge Densities for OH adsorbed to FM, AFM, and NM magnetic structures. Valence electrons are shown from (a-c) $-6 \mathrm{eV}$ to the Fermi energy and from (d-f) $-2 \mathrm{eV}$ to the Fermi energy. The scale has been narrowed to $25 \%$ of the total electronic states to improve the visibility of bonds. Contour lines indicate areas with the same energy and are consistent across structures. Blue and red indicate many and no electronic states, respectively. Some $\mathrm{U}$ and $\mathrm{N}$ atoms have been numbered for reference in the text. The numbering scheme is consistent across magnetic structures .86

Figure 4.6 Electron Localization Function (ELF) of OH adsorbed to (a) FM, (b) AFM, and (c) NM UN surfaces. Blue and red indicate high and low probability of finding a localized electron, respectively. Contour lines indicate areas with the same energy and are consistent across structures. 87

Figure 5.1 Starting atomic structures for (a) pure Mo, (b) the HTIR-Mo supercell used in DFT, (c) the HTIR-Mo supercell used in AIMD, (d) pure Nb, (e) 
the HTIR-Nb supercell used in DFT, and (f) the HTIR-Nb supercell used in AIMD. Purple, blue, light blue, orange, and red atoms represent Mo, $\mathrm{Nb}$, La, $\mathrm{P}$, and $\mathrm{O}$, respectively.

Figure 5.2 Radial Distribution Function in $\AA$ of HTIR-Nb AIMD equilibration at 20

${ }^{\circ} \mathrm{C}$ using $220 \mathrm{eV}$ and $520 \mathrm{eV}$ cutoff energies.

Figure 5.3 Experimental temperature profile as measured by two Type B TCs, voltage output by the HTIR-Nb - Pt and HTIR-Mo - Pt TCs during the heat treatment, and voltage output by the HTIR-Nb and HTIR-Mo thermoelements during the heat treatment. The heat soak at $1600{ }^{\circ} \mathrm{C}$ begins just after 6.5 hours.

Figure 5.4 Comparison of Seebeck coefficients for pure Mo and $\mathrm{Nb}$ from experiment $[21,22]$ and computation. 102

Figure 5.5 Comparison of the voltages obtained from experiment and DFT for (a) Mo and (b) Nb. The HTIR-Mo DFT structure contains $1.13 \mathrm{wt} . \% \mathrm{La}$ and the HTIR-Nb DFT structure contains 0.26 wt.\% P. 102

Figure 5.6 Temperature profile of the AIMD heat treatment performed on the HTIRMo and HTIR-Nb structures.

Figure 5.7 Voltage produced by (a) HTIR-Mo and (b) HTIR-Nb as measured in experiment, calculated in DFT, and calculated in AIMD. For the AIMD voltages, Seebeck coefficients obtained from an atomic structure at the given temperature were integrated over the full temperature range. For HTIR-Mo, the DFT structure contains 1.13 wt.\% La and the AIMD structures contain 1.13 wt.\% La 0.13 wt.\% O. For HTIR-Nb, the DFT structure contains $0.26 \mathrm{wt} . \% \mathrm{P}$ and the AIMD structures contain $0.26 \mathrm{wt} . \%$ P 0.14 wt.\% O.

Figure 5.8 Radial Distribution Functions for HTIR-Mo and HTIR-Nb for (a-b) DFT and AIMD $20^{\circ} \mathrm{C}$ and during the AIMD (c-f) heat ramp, (g-h) cool down, and (i-j) subsequent ramp corresponding to the heat up/cool down cycles in Figure 5.6. The peaks at $20^{\circ} \mathrm{C}$ before the heat ramp are numbered for clarity. A zoomed-in version of the heat ramp is provided (e-f) to better discern the structural evolution. Temperatures indicate the constant temperature simulation at which each RDF was obtained.

Figure 6.1 Diffusion coefficients of each surface exposed to $\mathrm{O} 2$.

Figure 6.2 Atomic structures of metals interacting with $\mathrm{O} 2$ after $10 \mathrm{ps}$ at $1600{ }^{\circ} \mathrm{C}$. Blue, purple, teal, and red atoms represent $\mathrm{Nb}, \mathrm{Mo}, \mathrm{Zr}$, and $\mathrm{O}$, respectively. 
Figure 6.3 Partial Charge Densities of metal surfaces under O2 after 0.5 and 1 ps at $1600{ }^{\circ} \mathrm{C}$. Electrons are resolved from $-2 \mathrm{eV}$ to the Fermi level. The scale has been narrowed to $20 \%$ of the total electronic states to improve the visibility of bonds. Contour lines indicate areas with the same energy and are consistent across structures. Blue and red indicate many and no electronic states, respectively .128

Figure 6.4 Electron Localization Functions of metal surfaces under $\mathrm{O}_{2}$ after 1 ps at $1600{ }^{\circ} \mathrm{C}$. Electrons are resolved from $-2 \mathrm{eV}$ to the Fermi level. Contour lines indicate areas with the same energy and are consistent across structures. Blue and red indicate a high and low probability of finding an electron, respectively.

Figure 6.5 Diffusion coefficients for (a) $\mathrm{O}$ and (b) $\mathrm{H}$ after each surface is exposed to $\mathrm{H}_{2} \mathrm{O}$. 130

Figure 6.6 Atomic structures of metals under 2 monolayers of $\mathrm{H}_{2} \mathrm{O}$ after $10 \mathrm{ps}$ at $1600{ }^{\circ} \mathrm{C}$. Blue, purple, teal, red, and white atoms represent $\mathrm{Nb}, \mathrm{Mo}, \mathrm{Zr}, \mathrm{O}$, and $\mathrm{H}$ respectively.

Figure 6.7 Nudged elastic band calculations for O intercalation into the Mo surface. In reaction pathway (I), O begins at a Mo-top site and transitions to an interstitial site. In reaction pathway (II), O begins at a hollow site and transitions to the interstitial site. The transition energies for pathway (I) are shown in (b) and pathway (II) are shown (c).

Figure 6.8 Electron Localization Functions of metal surfaces under $\mathrm{H}_{2} \mathrm{O}$ after 1 ps at $1600{ }^{\circ} \mathrm{C}$. Electrons are resolved from $-2 \mathrm{eV}$ to the Fermi level. Contour lines indicate areas with the same energy and are consistent across structures. Blue and red indicate high and low probability of finding a localized electron, respectively. 


\section{LIST OF ABBREVIATIONS}

\begin{tabular}{|c|c|}
\hline AIMD & $\mathrm{Ab}$ initio Molecular Dynamics \\
\hline AFM & Antiferromagnetic \\
\hline ATF & Accident Tolerant Fuel \\
\hline ATR & Advanced Test Reactor \\
\hline DFT & Density Functional Theory \\
\hline DOE & Department of Energy \\
\hline DOS & Density of States \\
\hline EDS & Energy Dispersive Spectroscopy \\
\hline FM & Ferromagnetic \\
\hline FP & Fission Product \\
\hline HPC & High Performance Computing \\
\hline HTGR & High Temperature Gas Reactor \\
\hline HTIR-TC & High Temperature Irradiation Resistant Thermocouple \\
\hline IPCC & Intergovernmental Panel on Climate Change \\
\hline LOCA & Loss of Coolant Accidents \\
\hline MD & Molecular Dynamics \\
\hline MSMSE & Micron School of Materials Science and Engineering \\
\hline NEB & Nudged Elastic Band \\
\hline NM & Nonmagnetic \\
\hline $\mathrm{NRC}$ & Nuclear Regulatory Commission \\
\hline
\end{tabular}




$\begin{array}{ll}\text { PCD } & \text { Partial Charge Density } \\ \text { RDF } & \text { Radial Distribution Function } \\ \text { SEM } & \text { Scanning Electron Microscopy } \\ \text { TREAT } & \text { Transient Reactor Test Facility } \\ \text { UN } & \text { Uranium Nitride } \\ \text { XRD } & \text { X-ray Diffraction }\end{array}$





\section{CHAPTER ONE: INTRODUCTION}

\subsection{Goal of the Dissertation}

The goal of this dissertation is to improve the understanding of two advanced nuclear energy concepts: uranium nitride (UN) and in-pile temperature sensors using first-principles methods. Better understanding of the properties and behaviors of these materials will contribute to developing materials solutions to improve their performance.

UN offers improvements to fuel economy and accident tolerance compared to the current standard nuclear fuel: uranium dioxide $\left(\mathrm{UO}_{2}\right)$. However, $\mathrm{UN}$ corrodes when exposed to water, which would lead to fission product release in the event of an accident. While UN corrosion has been studied experimentally, there is limited understanding of the prevalence of unreacted $\mathrm{N}$ and the electronic mechanisms of this electrochemical process. There is also no consensus on the magnetic structure of the UN surface. Since magnetic treatment will change the electronic structure, this may have a significant effect on corrosion properties. To meet these knowledge gaps, this work will identify atomic and electronic steps in corrosion initiation as well as the most stable magnetic structure of the UN surface.

In-pile temperature sensors known as High Temperature Irradiation Resistant Thermocouples (HTIR-TCs) are crucial to the characterization of advanced nuclear material performance. To best record the temperature in these advance nuclear material experiments, HTIR-TC development seeks to increase in-pile sensor lifetime and temperature resolution. This work develops a method to predict the performance of 
thermoelement materials with consideration of composition and temperature profiles. Once validated, this method can be used to predict performance changes due to material changes in-pile as well as identify thermoelement materials that may increase temperature resolution. The oxidation and corrosion of HTIR thermoelements is also investigated to better understand how reactor coolant may affect sensor lifetime.

Characterizing the atomic steps in corrosion and material evolution in-pile can be difficult experimentally. Using atomistic first-principles modeling allows for the precise selection of compositions, examination of atomic structural changes, and evaluation of electronic properties. The ability to resolve the electronic structure is especially crucial for these two areas of study as corrosion is an electrochemical process and HTIR-TCs rely on the Seebeck effect. Additionally, since no experimental parameterization is required, first-principles methods and the larger length scale methods they parameterize can have predictive capabilities. This work will ultimately help the development of nuclear power towards mitigating climate change.

\subsection{Background, Motivation, and Objectives}

To mitigate the projected effects of climate change and reduce our dependence on

fossil fuels, we need reliable, carbon-free energy. Though we cannot stop warming due to anthropogenic effects, we can still mitigate harm to human life and our planet. The Intergovernmental Panel on Climate Change (IPCC) projects that if we can limit continued warming to $1.5^{\circ} \mathrm{C}$ by 2052 , we can reduce the severity of extreme heat in inhabited regions, flooding, drought, sea level rise, and loss of biodiversity [1]. IPCC models in which net warming is limited to $1.5^{\circ} \mathrm{C}$ require reaching net zero $\mathrm{CO}_{2}$ emissions by 2050 . 
In the United States, electricity generation accounts for $27 \%$ of our greenhouse gas emissions. To cut back on these emissions, we need to rapidly increase our use of carbon-free power sources. Currently, nuclear power accounts for 55\% of our carbon-free power [2]. Nuclear power is not only carbon-free but also incredibly energy dense and reliable. One single fuel pellet can produce as much energy as 1 ton of coal, 149 gallons of oil, or 17,000 cubic feet of natural gas. This high energy density means nuclear power plants only need to be refueled about every 2 years and as such are able to operate over $90 \%$ of the time.

To make nuclear as viable a power source as possible, we need to maximize power density and safety. In the wake of the Fukushima-Daiichi accident, the nuclear field has focused on Accident Tolerant Fuel (ATF) concepts [3-5]. ATF materials are designed to maintain or improve upon the current fuel materials, while slowing the rate of fuel rod degradation and thus increasing time for accident response.

Before we can use ATF concepts in nuclear power plants, we need to know how they will behave in-pile. This requires a series of experimental irradiation campaigns, each of which can take a decade [6]. The Nuclear Regulatory Commission (NRC) has recognized that mechanistic modeling can be used to reduce the number of necessary experiments and also considered modeling as a component for qualifying advanced reactor concepts $[7,8]$. An example of materials models that can together be used for multi-scale mechanistic modeling are shown in Figure 1.1.

Developing mechanistic or physics-based engineering scale models requires accurate insight and input parameters from the lower length scale models, i.e. atomistic methods such as Density Functional Theory (DFT) and Molecular Dynamics (MD). It is 
thus the responsibility of atomistic modelers to produce accurate data as the foundation for expediting the advanced nuclear fuel material pipeline. Developing a physics-based method is crucial to obtain predictive capabilities. Conversely, with an entirely empirical method, only materials with significant corresponding experimental data could be modeled. This would circle back to the requirement of decades long irradiation campaigns, which would greatly hinder the goal to achieve net-zero carbon emissions by 2050.

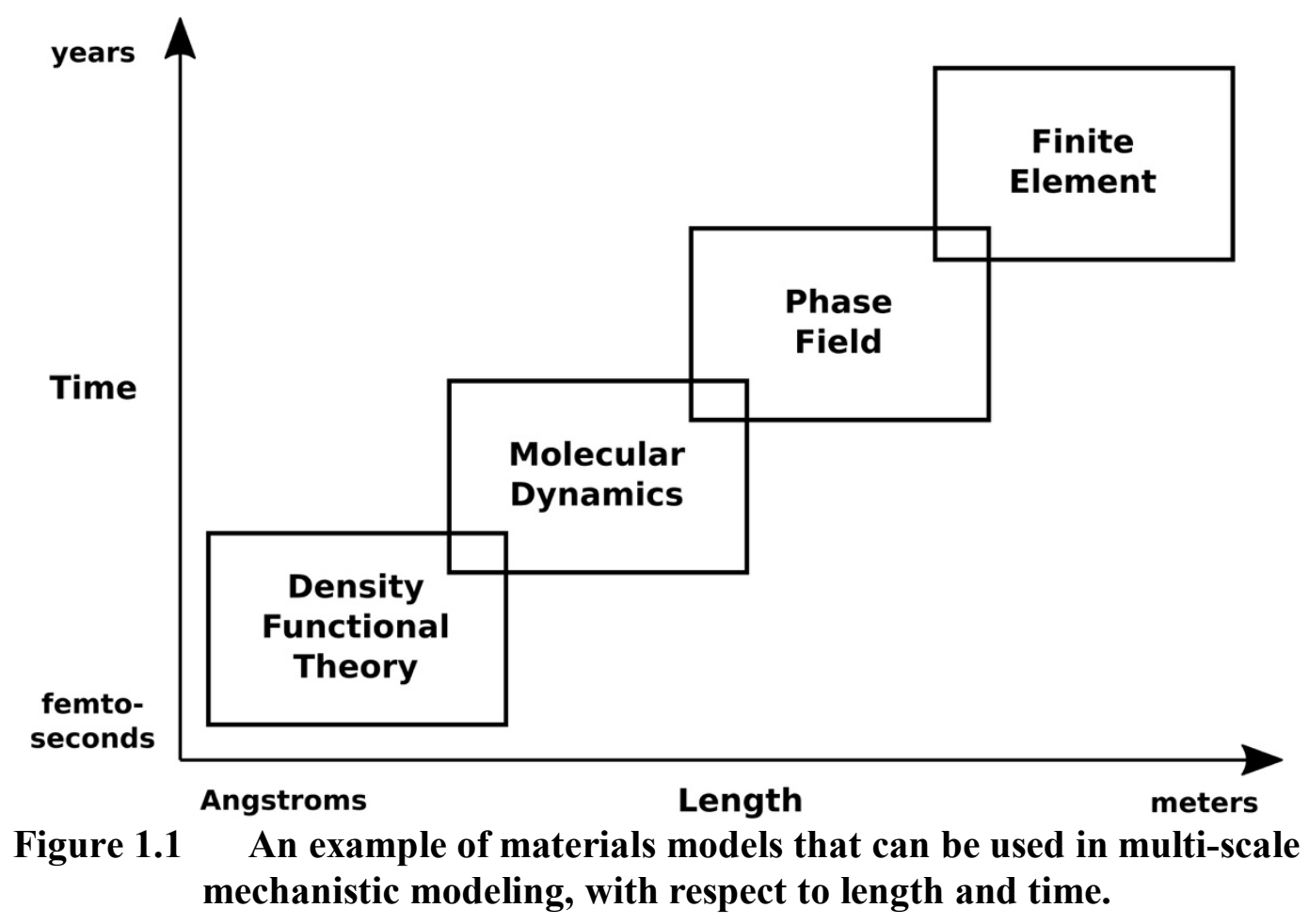

BISON is a finite element-based engineering scale code capable of investigating in-pile behavior such as swelling, fracture, and creep in nuclear reactors. While BISON was originally developed to study $\mathrm{UO}_{2}$, by changing the physics-based parameters the code can be modified to study new fuel forms [9]. Recent work by Cooper et al. [9] demonstrated how DFT data can ultimately lead to parameters for BISON modules. First, 
DFT was used to develop extensive data sets including formation energies, binding energies, and activation energies for interstitial, vacancy, and Xe defects in $\mathrm{UO}_{2}[10]$. This DFT data along with entropies and attempt frequencies obtained from Molecular Dynamics (MD) was used to parameterize a cluster dynamics model [11]. The cluster dynamics model was then used to relate Xe diffusivity to temperature for both $\mathrm{UO}_{2}$ and a candidate fuel form: $\mathrm{Cr}_{2} \mathrm{O}_{3}$ doped $\mathrm{UO}_{2}$. Using an Arrhenius fit, the cluster dynamics results were used to obtain the enthalpy and temperature parameters that were implemented in the BISON fission gas release module. BISON was then used to model the temperature, pressure, and ultimately fission gas release of an experimental scenario. The BISON results were in good agreement with in-reactor experimental measurements, thus validating the method.

In addition to parameterizing engineering models, parameters derived from atomistic models can reduce the uncertainty in the engineering models. For the U-Zr fuel form, Beeler et al. used MD to calculate surface tension in order to parameterize the BISON swelling module [12]. Surface tension directly influences fission gas bubble morphology and evolution within the fuel, which in turn affects the kinetics of swelling. The BISON swelling model can be tuned to match experiment using both surface tension and bubble number density parameters. By informing the surface tension parameter using lower length scale modeling, it was found the bubble number density needed to be significantly higher than previously assumed. This illustrates how atomistic models reduce the uncertainty in engineering scale models by refining physics-based parameters.

This dissertation uses atomistic modeling methods: DFT and Ab initio Molecular Dynamics (AIMD) to study two material systems of interest to ATF concepts and their 
development: ATF candidate uranium nitride (UN) and in-pile temperature sensors known as High Temperature Irradiation Resistant Thermocouples (HTIR-TCs).

Background information on each material system as well as dissertation deliverables are discussed in the following two sections.

\subsubsection{Uranium Nitride (UN)}

UN has several desirable properties for improving upon the accident tolerance of the current fuel: uranium dioxide $\left(\mathrm{UO}_{2}\right)$. Two key accident scenarios are Loss of Coolant Accidents (LOCA) and Radioactivity Initiated Accidents (RIA). During a LOCA, reactor temperatures can climb to $1500^{\circ} \mathrm{C}$ [4]. The current fuel rod assembly, composed of a $\mathrm{Zr}$ alloy cladding and $\mathrm{UO}_{2}$ fuel, is expected to rupture in the $700-1200^{\circ} \mathrm{C}$ range. Cladding can also rupture due to an RIA. When fuel rods are constructed, a gap or plenum initially exists between the fuel pellets and the cladding. However, during operation the fuel pellets begin to swell due to thermal expansion and fission product (FP) accumulation.

The cladding diameter creeps down due to water pressure $[13,14]$. After mechanical contact occurs, the cladding may rupture due to pressure during a power transient, especially if corrosive FPs build up at the interface [15].

With the above concerns, UN is a promising ATF candidate for its comparable melting temperature $\left(\sim 2850{ }^{\circ} \mathrm{C}\right)$, higher thermal conductivity $(23 \mathrm{~W} / \mathrm{mK}$ vs. $2.3 \mathrm{~W} / \mathrm{mK}$ at $1000 \mathrm{~K})$ and higher actinide density than $\mathrm{UO}_{2}\left(13.51 \mathrm{gU} / \mathrm{cm}^{3}\right.$ vs. $\left.9.66 \mathrm{gU} / \mathrm{cm}^{3}\right) \quad[16,17]$. These first two properties provide accident tolerance while the third increases power density. Since the fuel matrix and cladding serve as the first two barriers to FP release [3], their structural stability should be maintained as much as possible in the event of an accident. With a melting temperature of $2850^{\circ} \mathrm{C}, \mathrm{UN}$ is not susceptible to melting and 
subsequent release of FPs during an accident. Similarly, the higher thermal conductivity would reduce the thermal gradient, leading to both reduced pellet fragmentation and different geometry as the pellet swells. Pellets with the higher thermal conductivity have been shown to swell more like a barrel than the traditional hourglass shape, resulting in reduced interfacial stress between the cladding and the pellet $[13,18]$.

If cladding rupture occurs, the fuel will come into contact with the water or steam coolant. Unlike $\mathrm{UO}_{2}, \mathrm{UN}$ is susceptible to corrosion and would release FPs if the water degrades the UN pellets. Before UN can be used in power reactors, the understanding of this corrosion mechanism and materials solutions must be developed to prevent FP release.

The UN corrosion mechanism was first proposed in 1967 by Dell et al. [19] as:

$$
\mathrm{UN}+2 \mathrm{H}_{2} \mathrm{O} \rightarrow \mathrm{UO}_{2}+\mathrm{NH}_{3}+\frac{1}{2} \mathrm{H}_{2} \quad .
$$

However, this mechanism has proven to be incomplete. This initial study and numerous subsequent ones have reported residual $\mathrm{N}$ or $\mathrm{U} 2 \mathrm{~N} 3+\mathrm{x}$ present in the corrosion product [20-24]. Extensive studies have also continually identified an oxynitride phase of varying stoichiometry, $\mathrm{U}_{\mathrm{x}} \mathrm{O}_{\mathrm{y}} \mathrm{N}[20,22-24]$.

Experimental corrosion studies can be limited in the sense that in situ measurements can alter the observed mechanisms while measurements taken after corrosion experiments may miss temporal evolution of the corrosion mechanisms. UN corrosion studies with in situ data have examined the mechanism by continuously removing the gaseous species for identification by titration [19], gas chromatography [20], or mass spectrometry [25]. This method may alter the corrosion process as it is unknown whether such species are reaction products or intermediates. Conversely, 
studies that perform X-ray Diffraction (XRD), energy dispersive spectroscopy (EDS), and/or scanning electron microscopy (SEM) on the final product cannot reveal temporal information about the mechanism $[21,23,26]$.

DFT studies have been conducted to complement experiment in investigating UN corrosion and study atomic scale mechanisms $[16,27-30]$. While variations in experimental synthesis and fabrication can lead to varying density, purity, and geometries [24], DFT allows for precise selection of the reactant. Previous first-principles studies of $\mathrm{UN}$ corrosion have limited their study of corrosion initiation to single $\mathrm{O}$ and $\mathrm{H}$ adsorption [27-29]. While Bo et al. studied up to four water molecules at a $2 \times 2$ surface area (4 U atoms in the terminating layer), their interpretation was limited to pathways for water dissociation and adsorption sites [16,30]. There remains a significant knowledge gap in how water adsorption leads to oxynitride and $\mathrm{UO} 2$ formation and why residual $\mathrm{N}$ remains unreacted. In this dissertation, the first deliverable seeks to improve the understanding of UN corrosion initiation.

Objective 1: Identify atomistic and electronic corrosion initiation mechanisms of UN under full monolayer water coverage. By relaxing a full monolayer (defined as one adsorbate to one surface $\mathrm{U}$ atom) of water at the UN surface using DFT, further insight into UN corrosion initiation should be revealed. First, atomistic mechanisms reveal how water begins to alter the UN surface structure. A fundamental understanding of this atomistic mechanism can better aid experiment in identifying materials solutions. For example, DFT can model the UN surface with incorporation of a candidate additive for improving corrosion resistance and simulate how the additive may change corrosion progression. An ideal additive will hinder the atomistic structural changes water causes 
on the pure UN surface. Second, as an electrochemical process, an evolution of electron localization often plays a role in corrosion mechanisms. Mapping the electronic profiles in corrosion initiation provides further insight into how corrosion progresses and what an additive may seek to inhibit.

As mentioned previously, predictive larger length scale models rely on accurate DFT data. However, DFT studies of UN corrosion have not reached a consensus on how the magnetic structure of the UN surface should be treated on the atomic level. Experimentally, UN bulk has a type 1 antiferromagnetic (AFM) structure consisting of (100) planes alternating between all spin-up and all spin-down [31]. Using DFT, ferromagnetic (FM) treatment yields the most energetically favorable UN surfaces $[28,32,33]$. DFT studies with FM treatment $[27,28,34]$ have also cited an experimental magnetic susceptibility study reporting $17 \mathrm{~nm}$ UN crystallites as FM [35]. However, a more recent synchrotron x-ray study reported an epitaxially grown $70 \mathrm{~nm}$ UN film as AFM [36]. One DFT study chose to treat the UN surface as AFM like UN bulk [30], while another reported nonmagnetic treatment was appropriate for total energy calculations [29].

DFT UN corrosion studies have also disagreed on whether including a so-called Hubbard U-term is necessary $[16,30,34]$. In standard DFT, the Hartree energy term describes the potential between each electron and the total electron density (see Eq. (2-4)). Since the total electron density includes all electrons, this leads to each electron being repulsed by itself. This self-interaction error can cause electrons to over delocalize, which can lead to poor descriptions of properties in strongly correlated materials. Studies including elements with $\mathrm{d}$ and f electrons have counteracted this delocalization with the 
inclusion of an additional potential energy term, the Hubbard U-term [37]. For UN, Gryaznov et al. found that by increasing the effective U-term to $1.65 \mathrm{eV}$, the most energetically favorable magnetic structure transitioned from FM to AFM [38]. None of the DFT studies have yet clearly addressed if the inclusion of the Hubbard U-term will cause a change in favorability of magnetic structures of the UN surface. Towards ensuring accurate DFT findings for UN corrosion, the magnetic structure of the UN surface should be investigated with consideration of the Hubbard U-term.

Objective 2: Identify the most energetically favorable magnetic structure of the UN surface with consideration of the Hubbard U-term. As magnetic structure directly relates to the electrons of a material, magnetic treatment may significantly affect bonding, adsorption energies, and electronic profiles. As modeling continues to aid experiment towards understanding UN corrosion mechanisms and finding corrosion-resistant additives, the electronic mapping must be accurate. Determining the most energetically favorable magnetic structure while correcting for the self-interaction error will contribute to the accuracy of future DFT studies and of parameterization of larger-length scale UN studies.

\subsubsection{High Temperature Irradiation Resistant Thermocouples (HTIR-TCs)}

To test ATF materials, irradiation tests are conducted by inserting the materials into research reactors including the Advanced Test Reactor (ATR) and the Transient Reactor Test (TREAT) facility [39]. While power reactors operate at around $300{ }^{\circ} \mathrm{C}$, research reactors typically range from 900 to $1500{ }^{\circ} \mathrm{C}$ [40] with interest to go as high as $1800{ }^{\circ} \mathrm{C}[41]$. These research reactor temperatures allow for materials to be studied under LOCA conditions as well as next generation (Generation IV) nuclear reactor 
temperatures. During these tests, it is critical to accurately and dependably know the temperatures that the ATF materials are subjected to. For example, the margin test in the Advanced Gas Reactor (AGR) experiments is designed to demonstrate a margin between the highest temperature that fuel could reach during operation in a High Temperature Gas Reactor (HTGR) and the temperature the fuel will fail at [42].

Commonly, passive temperature sensors such as melt wires or paint spots are used to determine the maximum temperature reached [40]. However, these sensors provide no information on the temporal evolution of the temperature, which is necessary for both developing the understanding of candidate materials and validating predictive models. In order to obtain in-pile temperature data, robust instrumentation is needed that can endure both the irradiation and high temperatures that research reactors employ to simulate an accident.

Thermocouples (TCs) can provide real-time temperature data by utilizing the Seebeck effect. As illustrated in Figure 1.2, two dissimilar metals or thermoelements joined at one junction will produce a voltage, E, proportional to the temperature difference, $\mathrm{T}_{1}-\mathrm{T}_{0}$ :

$$
E=\int_{T_{0}}^{T_{1}}\left(\sigma_{+}-\sigma_{-}\right) d T \quad .
$$

where $\sigma_{+}$is the Seebeck coefficient of the positive thermoelement and $\sigma_{-}$is the Seebeck coefficient of the negative thermoelement. The positive and negative descriptors are relative, e.g. both thermoelements can have positive Seebeck coefficients and the one with greater magnitude is considered to be the positive leg. A TC can be calibrated by, for instance, measuring the voltage during a heat ramp in a furnace. Then when the TC is 
subsequently inserted into a new environment, e.g. a research reactor, the voltage reading can be converted back into temperature.

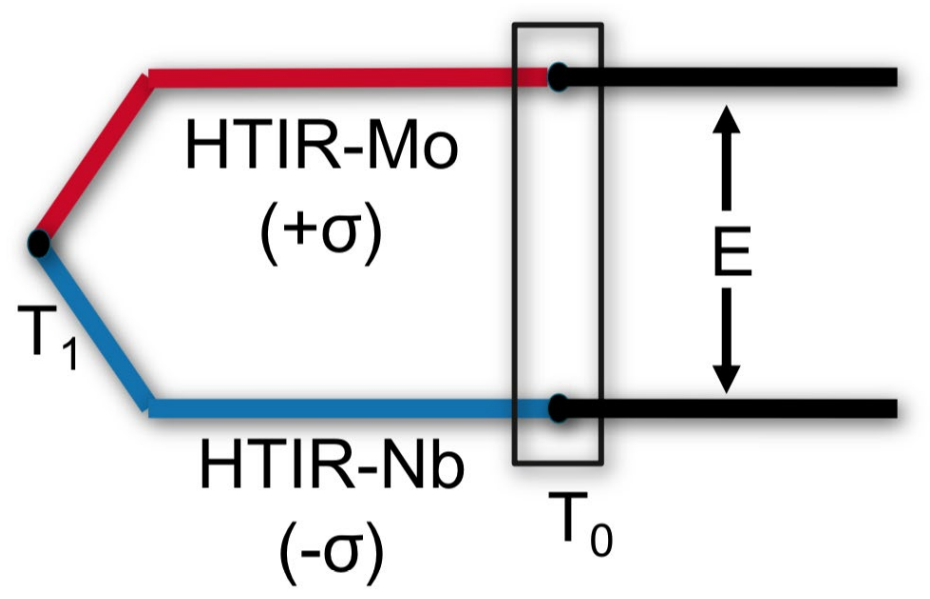

Figure 1.2 Schematic of a HTIR-TC consisting of two thermoelements: HTIRMo and HTIR-Nb which act as the positive and negative thermoelement, respectively. Each thermoelement has its own respective Seebeck coefficient, $\sigma$. One end of the thermocouple is placed into a reactor to measure a temperature, $T_{1}$, while the other end is held at a constant temperature, $T_{0}$, outside of the reactor. Due to the Seebeck effect, the thermocouple produces a voltage, $E$, proportional to temperature difference, $T_{1}-T_{0}$.

However, commercially available TCs struggle to meet the extreme conditions of a research reactor. Type B TCs (Pt-30\%Rh/Pt-6\%Rh) are rated for up to $1820{ }^{\circ} \mathrm{C}$, but $\mathrm{Rh}$, with a thermal cross section of 145 barns [43], is especially susceptible to transmutation. Conversely, TCs composed of Ni (4.6 barns thermal cross section) alloys: Type $\mathrm{K}$ (Chromel/Alumel) and Type $\mathrm{N}$ (Nicrosil/Nisil) are more resistant to transmutation but degrade over $1100{ }^{\circ} \mathrm{C}[41,44]$. Since TCs depend on material specific Seebeck coefficients, any changes in the thermoelement materials after calibration will lead to drift in the temperature reading.

To meet this need for thermocouples that can survive both the high temperature and irradiation of research reactors, Idaho National Laboratory (INL) has been 
developing High Temperature Irradiation Resistant Thermocouples (HTIR-TCs). During early testing in 2007, HTIR-TCs outperformed Type K and Type N TCs in a 4000+ furnace test at $1200^{\circ} \mathrm{C}[41]$. While the Type $\mathrm{K}$ and Type $\mathrm{N}$ TCs drifted by over $100^{\circ} \mathrm{C}$ or $8 \%$, the HTIR-TCs drifted by less than $20^{\circ} \mathrm{C}$ or $2 \%$.

A cross section of a standard TC is shown in Figure 1.3(a). The insulation, consisting of a crushable oxide, prevents physical contact between the two thermoelements before the desired temperature sensing junction. Mo and $\mathrm{Nb}$ were selected as thermoelement materials for HTIR-TCs due to their high melting temperatures (2610 and $2470{ }^{\circ} \mathrm{C}$, respectively) and low thermal cross sections (2.65 and 1.15 barns, respectively) [41]. Additionally, the thermoelements need to have minimal chemical reaction with the TC insulation. Zircaloy-4 was also considered as a prospective HTIR-thermoelement or sheath but had significant material interaction with the Al2O3 insulators. Pure Mo was found to recrystallize at $1200{ }^{\circ} \mathrm{C}$, leading to embrittlement and risk of TC failure. Mo doped with lanthanum oxide has been found to remain ductile even after heat treatment at $1600{ }^{\circ} \mathrm{C}$, provide the highest temperature resolution of studied Mo wires, and has thus been the positive HTIR-thermoelement of choice. $\mathrm{Zr}$ additions to $\mathrm{Nb}$ are expected to delay grain growth and as such $\mathrm{Nb}-1 \% \mathrm{Zr}$ has commonly been used as the sheathing material $[41,44]$. Mo additions to $\mathrm{Nb}$ may both delay recrystallization and increase temperature resolution $\left(\mathrm{V} /{ }^{\circ} \mathrm{C}\right)[44-46]$. Similarly, $\mathrm{Nb}$ additions to Mo may also increase its temperature resolution. However, early efforts in HTIR-TC development had limited success in fabricating developmental $\mathrm{Mo}$ and $\mathrm{Nb}$ alloys.

More recently, HTIR-TC development has focused on optimizing heat treatment and calibration [47-49]. After these optimizations, in 2019 HTIR-TCs were able to read 
$1247^{\circ} \mathrm{C}$ in a furnace over $1000+$ hours with a maximum of $0.6 \%$ or $5{ }^{\circ} \mathrm{C}$ drift with reference to the Type B TCs [49]. The current record holding in-pile HTIR-TC as of 2020 was able to continue reading $1450-1500{ }^{\circ} \mathrm{C}$ for 85 days in ATR without observable drift [42]. Any drift that occurred is difficult to quantify as a HTIR-TC is currently the most accurate way to read the true temperature inside a research reactor.

(a) Traditional TC build

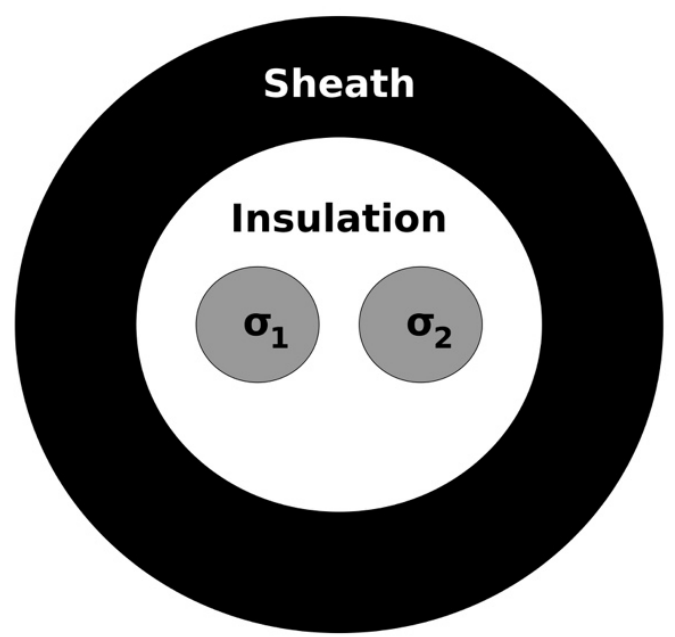

(b) Coaxial TC build

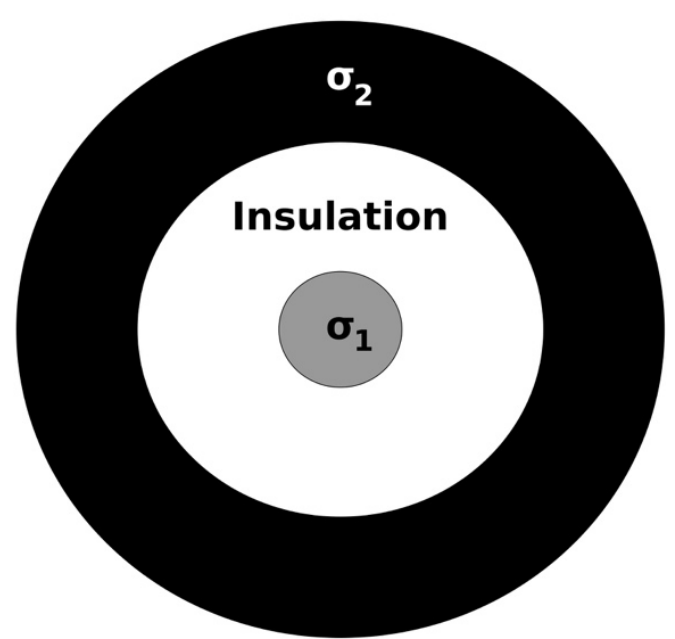

Figure 1.3 Cross sections of (a) the traditional thermocouple build and (b) the coaxial thermocouple build including two thermoelements: $\sigma_{1}$ and $\sigma_{2}$.

As development of HTIR-TCs continues, further methods of improving temperature resolution and increasing in-pile lifetime are being investigated. Improving HTIR-TC performance will benefit from an improved understanding of the effect of additive elements and temperature on Seebeck coefficients. This leads to the third deliverable.

Objective 3: Develop a method that can predict the Seebeck coefficient of a thermoelement based on composition and heat treatment. Following Eq. (1-2), the voltage reading of a TC can be calculated using the Seebeck coefficients of its thermoelements. It is crucial that the method be composition specific and sensitive to additives on the 
order of $1 \mathrm{wt} . \%$. Likewise, as HTIR-TCs are subjected to heat treatments and varying temperature profiles in-pile, the method should take into consideration temperature effects. If successfully validated, the method could be used to isolate the effects of dopants or alloying elements and temperature profile on HTIR-TC performance.

The predictive power could also be extended to both performance scenarios and screening developmental thermoelement compositions. The effect of compositional changes from irradiation including transmutation, vacancies, and interstitials could be predicted. In the coaxial build (Figure 1.3(b)), one of the thermoelements may be exposed to the reactor coolant. This could lead to oxidation and $\mathrm{H}$ uptake, and such compositional changes could also be investigated using the method. Finally, due to the cost and time investment of fabricating non-commercially available alloys, a predictive method could offer guidance on which alloys to pursue for higher temperature resolutions.

During the selection of optimal HTIR-thermoelements, Mo and Nb have been noted to be susceptible to oxidation [41]. Corrosion has also long been a problem in light water reactors, which may be accelerated at research reactor temperatures. ATR and TREAT use water and air as coolant, respectively. Deliverable four works towards evaluating the effect of air and water coolant on HTIR-TC lifetime.

Objective 4: Determine the extent of oxidation and corrosion risk in HTIRthermoelement materials. Identifying energy barriers to oxidation and corrosion of HTIRthermoelement materials could help evaluate if interaction with coolant is a contributing factor to HTIR-TC failure. If an oxidation- and corrosion-resistant HTIR-TC thermoelement is identified, it could be pursued as the sheath material or outer thermoelement in a coaxial TC build. 


\section{Outline of This Dissertation}

This dissertation is organized as follows. Chapter 2 describes the computational methods used for each of the material systems. Chapters 3 through 4 cover UN work. Chapter 3 addresses Object 1 . The better understood zirconium ( $\mathrm{Zr}$ ) corrosion mechanism is used as a reference to investigate $\mathrm{UN}$ corrosion. Specifically, the $\mathrm{Zr}$ corrosion mechanism is used to validate the ability of electronic structure mapping to elucidate mechanism steps. Insights gained from atomistic structural changes and electronic structure maps during the initial stage of corrosion are discussed. In Chapter 4, the magnetic structure of the UN (100) surface is investigated following Objective 2. The most stable magnetic treatment with consideration of the Hubbard U-term is identified. The effect of magnetic structure on stability, adsorption energy, bond lengths, bond angles, and electronic structure is discussed. Chapters 5 and 6 cover HTIR-TCs. In Chapter 5, a model methodology using atomic structure to predict sensor performance is presented following Objective 3. The effects of additives and temperature on HTIR-TC performance are also discussed. In Chapter 6, the corrosion and oxidation mechanisms of HTIR-TC materials exposed to water and air are investigated following Objective 4. The changes in electronic profiles and energy barriers to corrosion and oxidation are discussed. Finally, conclusions are given in Chapter 7. 


\section{References}

[1] Intergovernmental Panel on Climate Change, Headline Statements from the Summary for Policymakers, 2018.

[2] N.E. Institute, Nuclear Energy: Just the Facts, (2020) 1-36. https://www.nei.org/resources/fact-sheets/nuclear-energy-just-the-facts.

[3] Z. Duan, H. Yang, Y. Satoh, K. Murakami, S. Kano, Z. Zhao, J. Shen, H. Abe, Current status of materials development of nuclear fuel cladding tubes for light water reactors, Nucl. Eng. Des. 316 (2017) 131-150. doi:10.1016/J.NUCENGDES.2017.02.031.

[4] S.J. Zinkle, K.A. Terrani, J.C. Gehin, L.J. Ott, L.L. Snead, Accident tolerant fuels for LWRs: A perspective, J. Nucl. Mater. 448 (2014) 374-379. doi:10.1016/j.jnucmat.2013.12.005.

[5] K.A. Gamble, G. Pastore, M.W.D. Cooper, D. Andersson, ATF material model development and validation for priority fuel concepts L3 : FMC . FUEL . P19 . 06, 2019.

[6] J.A. Aguiar, A.M. Jokisaari, M. Kerr, R. Allen Roach, Bringing nuclear materials discovery and qualification into the 21st century, Nat. Commun. 11 (2020) 1-3. doi:10.1038/s41467-020-16406-2.

[7] U.S.N.R. Commission, PROJECT PLAN TO PREPARE THE U.S. NUCLEAR REGULATORY COMMISSION FOR EFFICIENT AND EFFECTIVE LICENSING OF ACCIDENT TOLERANT FUELS, 2018. https://www.nrc.gov/docs/ML1826/ML18261A414.pdf (accessed April 26, 2019).

[8] U.S.N.R. Commission, Approach for Code Development in Support of NRC's Regulatory Oversight of Non-Light Water Reactors, (2020).

[9] M.W.D. Cooper, G. Pastore, Y. Che, C. Matthews, A. Forslund, C.R. Stanek, K. Shirvan, T. Tverberg, K.A. Gamble, B. Mays, D.A. Andersson, Fission Gas Diffusion and Release for Cr2O3-Doped UO2 : From the Atomic to the Engineering Scale, J. Nucl. Mater. (2020) 152590. doi:10.1016/j.jnucmat.2020.152590. 
[10] R. Perriot, C. Matthews, M. Cooper, B.P. Uberuaga, C.R. Stanek, D.A.

Andersson, Atomistic modeling of out-of-pile xenon diffusion by vacancy clusters in UO2, J. Nucl. Mater. 520 (2019) 96-109.

doi:10.1016/J.JNUCMAT.2019.03.050.

[11] C. Matthews, R. Perriot, M.M.D. Cooper, C.R. Stanek, D.A. Andersson, Cluster dynamics simulation of xenon diffusion during irradiation in UO2, J. Nucl. Mater. (2020) 152326. doi:10.1016/j.jnucmat.2020.152326.

[12] B. Beeler, A. Casagranda, L. Aagesen, Y. Zhang, S. Novascone, Atomistic calculations of the surface energy as a function of composition and temperature in $\gamma \mathrm{U}-\mathrm{Zr}$ to inform fuel performance modeling, J. Nucl. Mater. 540 (2020) 152271. doi:10.1016/j.jnucmat.2020.152271.

[13] B. Michel, J. Sercombe, G. Thouvenin, A new phenomenological criterion for pellet-cladding interaction rupture, Nucl. Eng. Des. 238 (2008) 1612-1628. doi:10.1016/j.nucengdes.2008.01.012.

[14] N. Marchal, C. Campos, C. Garnier, Finite element simulation of Pellet-Cladding Interaction (PCI) in nuclear fuel rods, Comput. Mater. Sci. 45 (2009) 821-826. doi:10.1016/j.commatsci.2008.10.015.

[15] P. Jacques, F. Lefebvre, C. Lemaignan, Deformation-corrosion interactions for $\mathrm{Zr}$ alloys during I-SCC crack initiation: Part I: Chemical contributions, J. Nucl. Mater. 264 (1999) 239-248. doi:10.1016/S0022-3115(98)00501-7.

[16] T. Bo, J.-H. Lan, Y.-J. Zhang, Y.-L. Zhao, C.-H. He, Z.-F. Chai, W.-Q. Shi, Adsorption and dissociation of $\mathrm{H} 2 \mathrm{O}$ on the (001) surface of uranium mononitride: energetics and mechanism from first-principles investigation, Phys. Chem. Chem. Phys. 18 (2016) 13255-13266. doi:10.1039/c6cp01175f.

[17] J. Choi, B. Ebbinghaus, T. Meier, J. Ahn, Development (LDRD) on Monouranium Nitride Fuel Development for SSTAR and Space Applications, 2006. doi: $10.2172 / 877887$.

[18] H.S. Lee, D.J. Kim, D.S. Kim, D.R. Kim, Evaluation of thermomechanical behaviors of UO2-5 vol\% Mo nuclear fuel pellets with sandwiched configuration, J. Nucl. Mater. 539 (2020) 152295. doi:10.1016/j.jnucmat.2020.152295. 
[19] R.M. Dell, V.J. Wheeler, N.J. Bridger, Hydrolysis of uranium mononitride, Trans. Faraday Soc. 63 (1967) 1286. doi:10.1039/tf9676301286.

[20] S. Sugihara, S. Imoto, Hydrolysis of Uranium Nitrides, J. Nucl. Sci. Technol. 6 (1969) 237-242. doi:10.1080/18811248.1969.9732878.

[21] G.A.R. Rao, S.K. Mukerjee, V.N. Vaidya, V. Venugopal, D.D. Sood, Oxidation and hydrolysis kinetic studies on UN, J. Nucl. Mater. 185 (1991) 231-241. doi:10.1016/0022-3115(91)90340-D.

[22] M. Jolkkonen, P. Malkki, K. Johnson, J. Wallenius, Uranium nitride fuels in superheated steam, J. Nucl. Sci. Technol. 54 (2017) 513-519. doi:10.1080/00223131.2017.1291372.

[23] J.K. Watkins, D.P. Butt, B.J. Jaques, Microstructural degradation of UN and UNUO2 composites in hydrothermal oxidation conditions, J. Nucl. Mater. 518 (2019) 30-40. doi:10.1016/J.JNUCMAT.2019.02.027.

[24] J.K. Watkins, A. Gonzales, A.R. Wagner, E.S. Sooby, B.J. Jaques, Challenges and opportunities to alloyed and composite fuel architectures to mitigate high uranium density fuel oxidation: Uranium mononitride, J. Nucl. Mater. (2021) 153048. doi:10.1016/j.jnucmat.2021.153048.

[25] M. Jolkkonen, P. Malkki, K. Johnson, J. Wallenius, Uranium nitride fuels in superheated steam, J. Nucl. Sci. Technol. 54 (2017) 513-519. doi:10.1080/00223131.2017.1291372.

[26] D.A. Lopes, S. Uygur, K. Johnson, Degradation of $\mathrm{UN}$ and $\mathrm{UN}-\mathrm{U}_{3} \mathrm{Si}_{2}$ pellets in steam environment, J. Nucl. Sci. Technol. 54 (2017) 405-413. doi:10.1080/00223131.2016.1274689.

[27] D. Bocharov, D. Gryaznov, Y.F. Zhukovskii, E.A. Kotomin, Ab initio modeling of oxygen impurity atom incorporation into uranium mononitride surface and subsurface vacancies, J. Nucl. Mater. 416 (2011) 200-204. doi:10.1016/j.jnucmat.2010.11.090.

[28] D. Bocharov, D. Gryaznov, Y.F. Zhukovskii, E.A. Kotomin, Ab initio simulations of oxygen interaction with surfaces and interfaces in uranium mononitride, J. Nucl. Mater. 435 (2013) 102-106. doi:10.1016/j.jnucmat.2012.12.031. 
[29] R.-S. Li, X. Peng, W. Fei, M. Wen-Yan, Density functional theory study of H, C and $\mathrm{O}$ chemisorption on UN(001) and (111) surfaces, Nucl. Sci. Tech. 25 (2014) 050502-10. doi:10.13538/j.1001-8042/nst.25.050502.

[30] T. Bo, J.-H. Lan, Y.-L. Zhao, C.-H. He, Z.-F. Chai, W.-Q. Shi, First-principles study of water reacting with the (110) surface of uranium mononitride, J. Nucl. Mater. 492 (2017) 244-252. doi:10.1016/J.JNUCMAT.2017.05.026.

[31] N.A. Curry, An investigation of the magnetic structure of uranium nitride by neutron diffraction, Proc. Phys. Soc. 86 (1965) 1193-1198. doi:10.1088/0370$1328 / 86 / 6 / 304$.

[32] Y.F. Zhukovskii, D. Bocharov, E.A. Kotomin, Chemisorption of a molecular oxygen on the UN(0 0 1) surface: Ab initio calculations, J. Nucl. Mater. 393 (2009) 504-507. doi:10.1016/j.jnucmat.2009.07.010.

[33] Y.F. Zhukovskii, D. Bocharov, E.A. Kotomin, R.A. Evarestov, A.V. Bandura, First principles calculations of oxygen adsorption on the UN(001) surface, Surf. Sci. 603 (2009) 50-53. doi:10.1016/j.susc.2008.10.019.

[34] J. Chen, Z. Long, R. Qiu, Y. Hu, B. Ao, K. Liu, Molecular reactions and oxidation corrosion on UN (001) surface under exposure to environment gases: A DFT study, J. Nucl. Mater. 533 (2020) 152095. doi:10.1016/j.jnucmat.2020.152095.

[35] D. Rafaja, L. Havela, R. Kužel, F. Wastin, E. Colineau, T. Gouder, Real structure and magnetic properties of UN thin films, J. Alloys Compd. 386 (2005) 87-95. doi:10.1016/J.JALLCOM.2004.06.020.

[36] E.L. Bright, R. Springell, D.G. Porter, S.P. Collins, G.H. Lander, Synchrotron xray scattering of magnetic and electronic structure of $\mathrm{UN}$ andU2N3 epitaxial films, Phys. Rev. B. 100 (2019) 1-9. doi:10.1103/PhysRevB.100.134426.

[37] D.S. Sholl, J.A. Steckel, Density Functional Theory: A Practical Introduction, 2009.

[38] D. Gryaznov, E. Heifets, E. Kotomin, The first-principles treatment of the electron-correlation and spin-orbital effects in uranium mononitride nuclear fuels, Phys. Chem. Chem. Phys. 14 (2012) 4482-4490. doi:10.1039/c2cp40297a. 
[39] Shannon Bragg-Sitton, Development of advanced accident-tolerant fules for commercial LWRs, Nucl. News. (2014) 83-91.

[40] B.G. Kim, J.L. Rempe, J. Villard, S. Solstad, Review Paper: Review of Instrumentation for Irradiation Testing of Nuclear Fuels and Materials, Radiat. Meas. Gen. Instrum. 176 (2011) 155-187. doi:10.13182/NT11-A13294.

[41] J.L. Rempe, D.L. Knudson, K.G. Condie, S.C. Wilkins, Thermocouples for HighTemperature In-Pile Testing, Nucl. Technol. 156 (2006) 320-331. doi:10.13182/NT06-A3794.

[42] A.J. Palmer, R.S. Skifton, M. Scervini, D.C. Haggard, W.D. Swank, Performance of Custom-Made Very High Temperature Thermocouples in the Advanced Gas Reactor Experiment AGR-5/6/7 during Irradiation in the Advanced Test Reactor, EPJ Web Conf. ANIMMA 2019. 225 (2020). doi:10.1051/epjconf/202022504010.

[43] S.F. Mughabghab, Thermal neutron capture cross sections resonance integrals and g-factors, 2003.

[44] J. Daw, J. Crepeau, J. Rempe, D. Knudson, K. Condie, C. Wilkins, Initial Results from Investigations to Enhance the Performance of High Temperature IrradiationResistant Thermocouples, J. Power Energy Syst. 2 (2008) 854-863. doi:10.1299/jpes.2.854.

[45] J.L. Rempe, D.L. Knudson, K.G. Condie, J.C. Crepeau, J.E. Daw, S.C. Wilkins, Options Extending the Applicability of High-Temperature Irradiation-Resistant Thermocouples, Nucl. Technol. 167 (2009) 169-177. doi:10.13182/NT09-14.

[46] R. Schley, G. Metauer, Thermocouples for measurements under conditions of high temperature and nuclear radiation, Temp. Its Meas. Control Sci. Ind. 5 (1982) 1109-1113.

[47] R. Skifton, J. Palmer, P. Calderoni, Optimization of heat treatment and calibration procedures for high temperature irradiation resistant thermocouples, Instrum. Sci. Technol. 46 (2017) 1-15. doi:10.1080/10739149.2017.1389754.

[48] R. Skifton, J. Palmer, K. Davis, P. Calderoni, E. Sikorski, D. Corbett, Summary of high temperature irradiation resistant thermocouple standardization tests, in: 11th Nucl. Plant Instrumentation, Control. Human-Machine Interface Technol. NPIC HMIT 2019, 2019. 
[49] R.S. Skifton, J. Palmer, L. Kurt, P. Calderoni, D. Corbett, E.L. Sikorski, Summary of High Temperature Irradiation Resistant Thermocouple Standarization Tests, in: 11th Nucl. Plant Instrumentation, Control Human-Machine Interface Technol., 2019. 


\section{CHAPTER TWO: METHODS}

\subsection{Density Functional Theory (DFT)}

DFT is used to obtain material properties at the atomistic scale. Commonly referred to as "first-principles" or "ab initio" methods, its strength lies in only requiring knowledge of the elements present in the system of interest and an educated guess for the atomic structure. The primary output of DFT is the total energy of a system which reveals how favorable or unfavorable, stable or unstable, exothermic or endothermic, a structure or reaction snapshot is. In addition to total energy, DFT obtains the wave functions and charge density of a system.

DFT starts from the Schrödinger time-independent wave equation:

$$
\widehat{H}(\vec{r}) \Psi(\vec{r})=E \Psi(\vec{r}) \quad, \quad \text { Eq. }(2-1)
$$

where $\widehat{H}$ is the Hamiltonian operator, $\Psi$ is the wave function, $E$ is the energy, and $\vec{r}$ is the electronic coordinates. As a result of the $\widehat{H}$ operating on the $\Psi$, we obtain the $E$ crucial to DFT studies. DFT has developed to efficiently ignore aspects that would have great computational cost with little effect on results at the atomistic scale including gravity and relativity [1]. Time is ignored as well, but, as we shall see, a great deal of mechanistic information can be gained by comparing energies of different atomistic configurations and by using the DFT - Molecular Dynamics (MD) hybrid known as Ab initio Molecular Dynamics (AIMD). 
The current standard version of the DFT Hamiltonian includes four terms: the non-interacting kinetic energy, external energy, Hartree energy, and exchange-correlation energy. The kinetic energy term is given as:

$$
E_{\text {kin }}=\frac{\hbar}{2 m} \sum_{i}^{n} \nabla^{2}
$$

The external energy, $E_{\text {ext }}$, corresponds to the potential, $U_{\text {ext }}$, on the electrons due to the atomic nuclei following:

$$
E_{\text {ext }}=\int U_{\text {ext }}(\vec{r}) \rho(\vec{r}) d \vec{r} \quad \text { Eq. }(2-3)
$$

As nuclei are much more massive than electrons, they are considered to be frozen while the electronic positions and orbitals are optimized. The nuclei positions are updated in a separate loop as discussed in more detail below.

In order to treat a complex interacting $n$-particle system, the system is converted to non-interacting system such that each electron sees the other electrons as an averaged potential or density, giving DFT its name. This energy is known as the Hartree energy:

$$
U_{H}(\vec{r})=\int \frac{\rho\left(\vec{r}^{\prime}\right)}{\left|\vec{r}-\vec{r}^{\prime}\right|} d \vec{r}^{\prime} \quad, \quad \text { Eq. }(2-4)
$$

where $\rho$ is the electron density, $\vec{r}$ is the position of an electron, and $\vec{r}^{\prime}$ is the position of the mean electron density in a mean-field approximation.

Extra care is taken to treat the exchange and correlation energies which correspond to electrons with same and different spin, respectively. While the exchangecorrelation energy, $U_{x c}$, accounts for the lowest magnitude of all the energy terms, the accuracy of DFT calculations hinges on this term. Two foundational $U_{x c}$ approximations are the Local Density Approximation (LDA) and Generalized Gradient Approximation (GGA) [1]. LDA is a functional of electron density: 


$$
E_{x c}^{L D A}=E_{x}^{L D A}[\rho(\vec{r})]+E_{c}^{L D A}[\rho(\vec{r})] \quad . \quad \text { Eq. (2-5) }
$$

Meanwhile, GGA is a functional of both the electron density and its gradient:

$$
E_{X C}^{G G A}=\int \rho(\vec{r}) \varepsilon_{X C}^{G G A}[\rho(\vec{r}), \nabla \rho(\vec{r})] d \vec{r} \quad . \quad \text { Eq. (2-6) }
$$

Since GGA includes both local and semi-local information on the electron density, it is generally used for the best accuracy. Advanced hybrid functionals also exist but often include empirical data and as such are not suited for calculations intended for predictive, mechanistic models. Combining each of these energy terms together results in the Kohn-Sham Hamiltonian:

$$
\widehat{H_{K S}}=E_{k i n}^{n o n}+U_{e x t}+U_{H}+U_{X C} .
$$

To achieve a non-interacting $n$-particle system, the wave functions are also converted to a linear combination of non-interacting orbitals, $\phi_{i}$. To obtain an interpretable physical meaning from $\phi_{i}$, they are squared to obtain the charge density:

$$
\rho(\vec{r})=\sum_{i}\left|\phi_{i}(\vec{r})\right|^{2} \quad . \quad \text { Eq. }(2-8)
$$

To solve the Kohn-Sham equation, DFT employs a Self-consistent Framework (SCF) as follows:

1. Create an educated ansatz for charge density using the respective element pseudopotentials for the system of interest.

2. Calculate Kohn-Sham Hamiltonian terms (Eq. (2-5)).

3. Solve the Kohn-Sham equations using direct diagonalization to obtain the Kohn-Sham orbitals, $\phi_{i}$.

4. Using the new Kohn-Sham orbitals, $\phi_{i}$, update the charge density $\rho(\vec{r})$.

5. Loop over steps 2 through 4 until the input and output Kohn Sham orbitals, $\phi_{i}$, are self-consistent. 
This marks the end of a single electronic loop. Note that the pseudopotentials commonly used in DFT differ from the all electron potentials for elements in that the core electrons are frozen along with the nuclei. This reduces computational cost while maintaining consideration of the valence electrons that will participate in our material properties of interest.

Once electronic convergence is met, the forces on each atom or nuclei are calculated. The force calculation is incredibly computationally inexpensive compared to Kohn-Sham orbital, eigenvalue, and charge density updates. Following the HellmannFeynman theorem, the force on an atom $I$ is given as:

$$
\overrightarrow{F_{I}}=-\frac{d E}{d \overline{r_{I}}} \quad .
$$

The atoms are then spatially moved in the negative direction of the energy gradient and a new electronic loop is started. Once the ionic steps also reach selfconsistency, we obtain our converged total energy and are able to start processing the data to investigate other properties.

Another method of reducing computational cost is the implementation of Periodic Boundary Conditions (PBCs). Since most solids can be represented by unit cells, we can model a single unit cell with PBCs in three dimensions. These images of adjacent cells will simulate an infinite solid. By understanding the PBCs implemented in our DFT code, we can model bulk material or surfaces. In the case of bulk simulations, a sufficiently large supercell needs to be selected to produce desirable concentrations of defects or additives. For surfaces, a vacuum is introduced in one direction such that each slab will not interact with its next image. The surface area in the unit cell must also be considered in studies of surface interactions such as corrosion. Too small of a surface area may 
produce a disproportionately large energy barrier to diffusion. For example, if we were to construct a slab with the surface area of a single $\mathrm{Nb}$ unit cell and investigate its interaction with $\mathrm{O}$, the $\mathrm{O}$ would need enough energy to enter every single surface interstitial site in the infinite $\mathrm{Nb}$ slab. Thus, a balance must be struck between a large enough unit cell to achieve reasonable energy barriers and preventing excessive computational cost.

In addition to unit cell choice and energy convergence criteria, the accuracy of a DFT simulation is dependent on the cutoff energy and number of reciprocal points or kpoints sampled. Once our basis set is selected, the eigenfunctions are given as:

$$
\chi_{j}(\vec{r})=\sum_{i}^{K} \alpha_{j i} \phi_{i} \quad, \quad \text { Eq. }(2-10)
$$

where $\alpha_{j i}$ are the expansion coefficients. To precisely match the wave function, the basis set could be expanded with $K$ going to infinity. However, we can determine an appropriate cutoff energy by means of a convergence test. To perform a convergence test, a series of nearly identical calculations are run while varying one parameter, such as cutoff energy, incrementally. Once the calculations are finished, a property of interest (e.g. total energy, lattice parameter, etc.) is compared across the calculations. Once the value plateaus or differs by a reasonable number, such as less than $0.1 \mathrm{eV}$, we can use the lower cutoff energy to achieve computationally efficient and accurate simulations.

Similarly, DFT accuracy is also related to k-point selection. This work utilizes the $\Gamma$-point method, in which reciprocal space is sampled either at or around a high symmetry point. The number of k-points in each direction must be inversely proportional to the unit cell dimensions. In the case of a surface calculation, more k-points are selected in the $\mathrm{x}$ and $y$ directions corresponding to the surface area while the vacuum direction $\mathrm{z}$ will have 
the lowest number of k-points. For example, the k-points for a surface can be set to $4 \times 4 \times 1$ to focus reciprocal sampling over the surface rather than the vacuum. The number of k-points can also be determined using a convergence test.

One drawback of DFT is the unphysical creation of self-interaction, that is the interaction of an electron with itself [2]. When DFT constructs the average electron gas that each electron "feels," all electrons are included. This means that each electron will also be repulsed by itself. This leads to over delocalization of electrons which can cause DFT calculations to yield inaccurate lattice parameters, band structures, magnetic ground states, and binding energies $[3,4]$. This DFT error leads to the greatest inaccuracies in materials with strongly localized, also known as correlated, electrons [2]. Coincidentally this means that actinides and transition metals, the basis of nuclear fuel and HTIR-TC material systems, respectively, require a self-interaction remedy. This is commonly treated by the addition of another potential energy term in the Hamiltonian known is the Hubbard +U term [4].

\subsection{Ab initio Molecular Dynamics (AIMD)}

In classical MD, simulations are governed by Newtonian mechanics. This can be represented as a Lagrangian [2]:

$$
L=K+U=\frac{1}{2} \sum_{i}^{3 N} m_{i} v_{i}^{2}+U\left(r_{i}, \cdots, r_{3 N}\right) \quad, \quad \text { Eq. }(2-11)
$$

where $U$ is constructed using MD potentials. Unlike in DFT, traditional MD potentials do not update during the progression of the calculation [1]. This contributes to the ability of MD to calculate many orders of magnitude more atoms than DFT. However, this requires the creation of MD potentials either empirically or using DFT results. Thus, there is a potential barrier to entry for studying material systems with no 
published MD potentials. The static potentials can also struggle to accurately model chemical reactions or systems such as surfaces where surface atom behavior varies from bulk atom behavior. While some advanced MD potentials such as ReaxFF can evolve dynamically, this is done at great computational cost and again must be parameterized extensively.

An incredibly powerful hybrid method known as AIMD gains the benefits of temporal evolution of atoms in MD and the first-principles capabilities of DFT by solving the potentials in Eq. (2-9) "on-the-fly" [2]. For this method, the $U$ term in the classical Lagrangian can be replaced the Kohn-Sham eigenfunctions:

$$
L=\frac{1}{2} \sum_{i}^{3 N} m_{i} v_{i}^{2}+E\left[\phi\left(r_{i}, \cdots, r_{3 N}\right)\right] \quad .
$$

In this work, AIMD allows for first-principles investigations to be extended to include both time and temperature.

\subsection{Methods Implemented in This Dissertation}

Once the atomic structure has been obtained from DFT or AIMD, several methods can be used to process the output data in order to investigate properties and behavior of the material systems. A flow chart of how the data was processed in this dissertation is shown in Figure 2.1. The benefits and/or information that can be investigated with each method is listed. Depending on the objective, the atomic structure was first optimized with DFT and/or AIMD. In some cases, DFT was used to optimize a structure before it was used in AIMD or to identify the most favorable incorporation site for an additive. In order to utilize some of the methods for an AIMD optimized structure, an electronic SCF had to be performed using DFT to obtain the wave functions and charge density with high accuracy. Once the atomic structures were optimized, a 
combination of methods in the "process output" category of Figure 2.1 was used to best meet the goals of the respective objective.

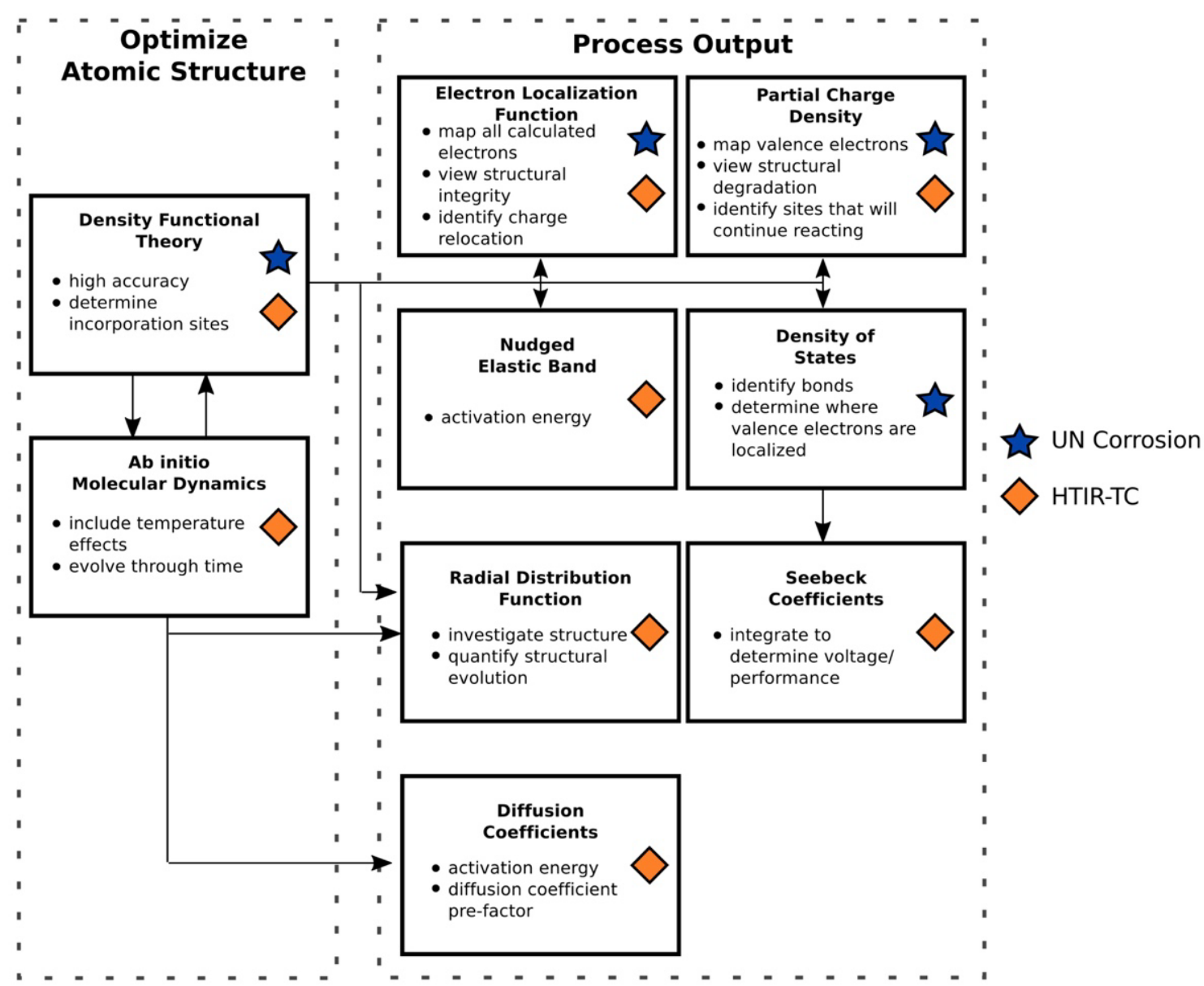

Figure 2.1 Methods used in this dissertation. Methods marked with blue stars and orange diamonds were used for the UN corrosion and HTIR-TC projects, respectively.

The electronic structures of the material systems can be analyzed using Density of States (DOS), Partial Charge Density (PCD), and the Electron Localization Function (ELF). DOS gives the number of electronic states with respect to energy. By referencing the Fermi energy, the populations of valence electrons and conduction electrons can be found immediately below and above the Fermi energy, respectively. This information can also be separated by element, summing all the states within a given distance from, for 
instance, each oxygen atom, summing all the states near a uranium atom, and so on. This separation by element is known as the Local Density of States (LDOS). An example LDOS is given in Figure 2.2. This LDOS shows that more valence electrons from -2 to 0 $\mathrm{eV}$ are localized to $\mathrm{U}$ than $\mathrm{N}$ and that bonding between the $\mathrm{UN}$ surface and an $\mathrm{O}$ adsorbate occurs around $-4 \mathrm{eV}$.

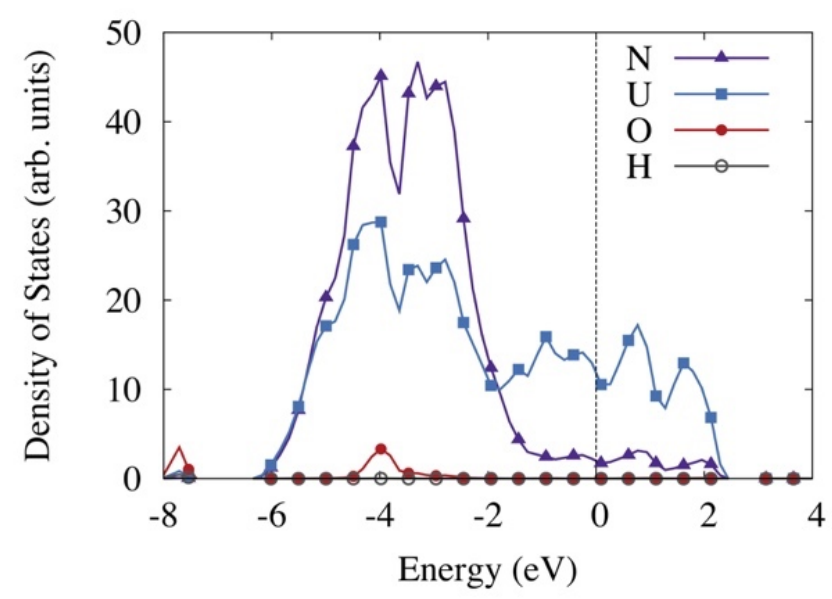

Figure 2.2 An example of Local Density of States (LDOS) for an OH adsorbed to a UN surface. The Fermi energy has been shifted to $0 \mathrm{eV}$ by convention.

The PCD, also known as band decomposed charge density, essentially maps a selected energy range from the DOS to the atomic structure. The PCD shown in Figure 2.3 maps the valence electrons from $-2 \mathrm{eV}$ to the Fermi energy onto the atomic structure of an $\mathrm{OH}$ adsorbed to a UN surface. Since the contour lines indicate areas with the same energy, they can be used as a visual aid to examine bonding. The contour lines indicate the bonds between $\mathrm{U} 1$ and its neighboring $\mathrm{U}$ and $\mathrm{N}$ atoms are weakened as compared to the bonds in the rest of the surface. The contour lines further indicate that of the U1 bonds with the rest of the surface, the bond with $\mathrm{U} 2$ is weakened the greatest since the contour lines no longer show a connecting region. 
$-2 \mathrm{eV}$ to $\mathrm{E}_{\mathrm{F}}$

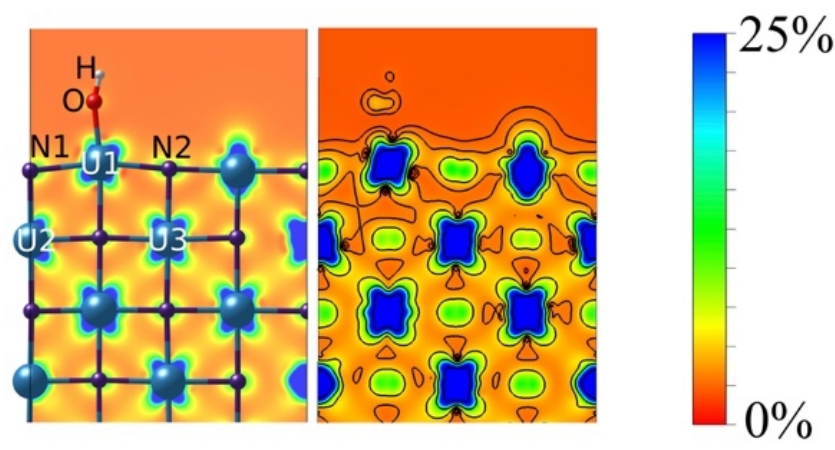

Figure 2.3 Partial Charge Density (PCD) from $-2 \mathrm{eV}$ to the Fermi energy for an $\mathrm{OH}$ adsorbed to a UN surface. The scale has been narrowed to $25 \%$ of the total electronic states to improve the visibility of bonds. Contour lines indicate areas with the same energy. Blue and red indicate many and no electronic states, respectively. Some $U$ and $N$ atoms have been numbered for reference in the text.

In contrast, the ELF sums over all the calculated electrons and determines the probability of finding an electron as a function of distance from a given electron with the same spin [5]. Instead of examining specific energy ranges, the ELF provides a more cohesive, mechanistic view. The calculation maps the electron population at a point to a number between 0 and 1, where 0 represents low probability of finding an electron, 1 represents high probability of finding an electron, and 0.5 refers to the population of a homogeneous electron gas. Polo et al. [6] reviewed the use of the ELF applied along an organic chemistry reaction pathway to explicitly determine electronic changes including bond forming and breaking, evolution of lone pairs, and transformation of double bonds to single bonds. An example ELF is shown in Figure 2.4. The blue $(\mathrm{ELF}=0.75)$ is localized to the adsorbed $\mathrm{OH}$ and above one of the $\mathrm{U}$ atoms in the terminating layer, indicating that there is the highest probability of finding an electron at. Similarly, the other terminating layer due to PBCs visible at the top of Figure 2.4 has some yellow or approximately 0.25 ELF character. Since lower ELF values represent a lower probability of finding a localized electron, the surface can be interpreted as positively charged. 


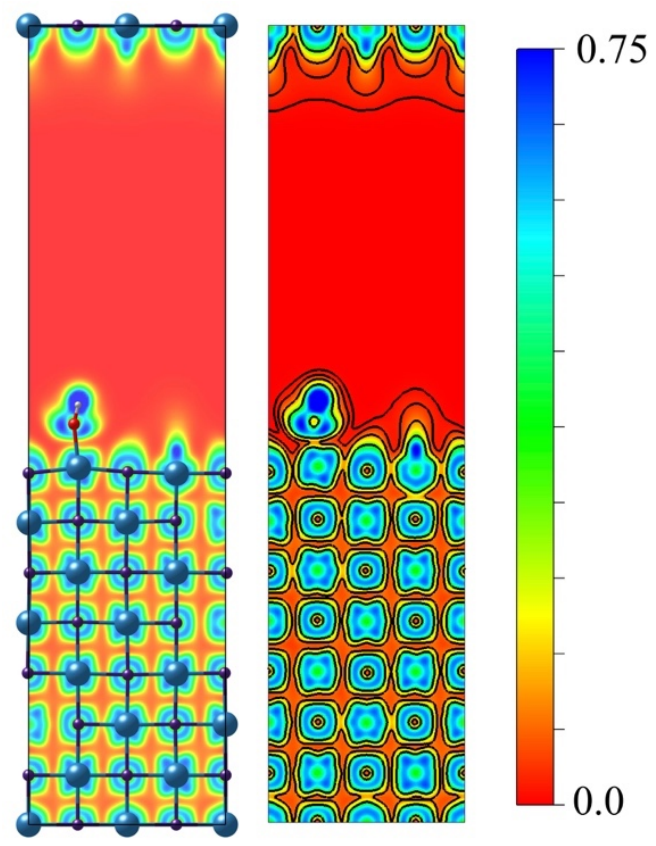

Figure 2.4 Example of Electron Localization Function (ELF) for an $\mathrm{OH}$ adsorbed to a UN surface. Blue and red indicate high and low probability of finding a localized electron, respectively. Contour lines indicate areas with the same energy.

Both the PCD and ELF output a three-dimensional map on top of the atomic structure. The examples (Figure 2.3 and Figure 2.4) show "slices" of these maps taken to examine the cross section on a particular plane. Using the LDOS, PCD, and ELF together allow us to identify bonding, zoom into a particular energy range and view the electrons on the structure, and examine the overall localization of electrons on the structure.

As we examine the atomic structure temporally, it is often helpful to use a quantitative method to interpret the structural changes. This can be accomplished the Radial Distribution Function (RDF), also sometimes referred to as the Pair Correlation Function (PCF). The RDF sums all atoms found at a given radial distance from a reference atom [1]. In a crystalline solid, the RDF consists of clearly defined peaks, indicating the number of atoms at the first-nearest neighbor distance, second-nearest neighbor distance, and so on. For amorphous or liquid structures, the RDF peaks are 
broader, indicating the greater disorder as compared to a crystalline material. An example of RDFs is given in Figure 2.5 which shows the disorder in the HTIR-Mo structure as it is heated up to $1600{ }^{\circ} \mathrm{C}$. As the structure heats, the peaks become broader with original peak 4 merging with peak 5,7 with 8,12 with 13 , and 15 with 16 .

\section{HTIR-Mo}

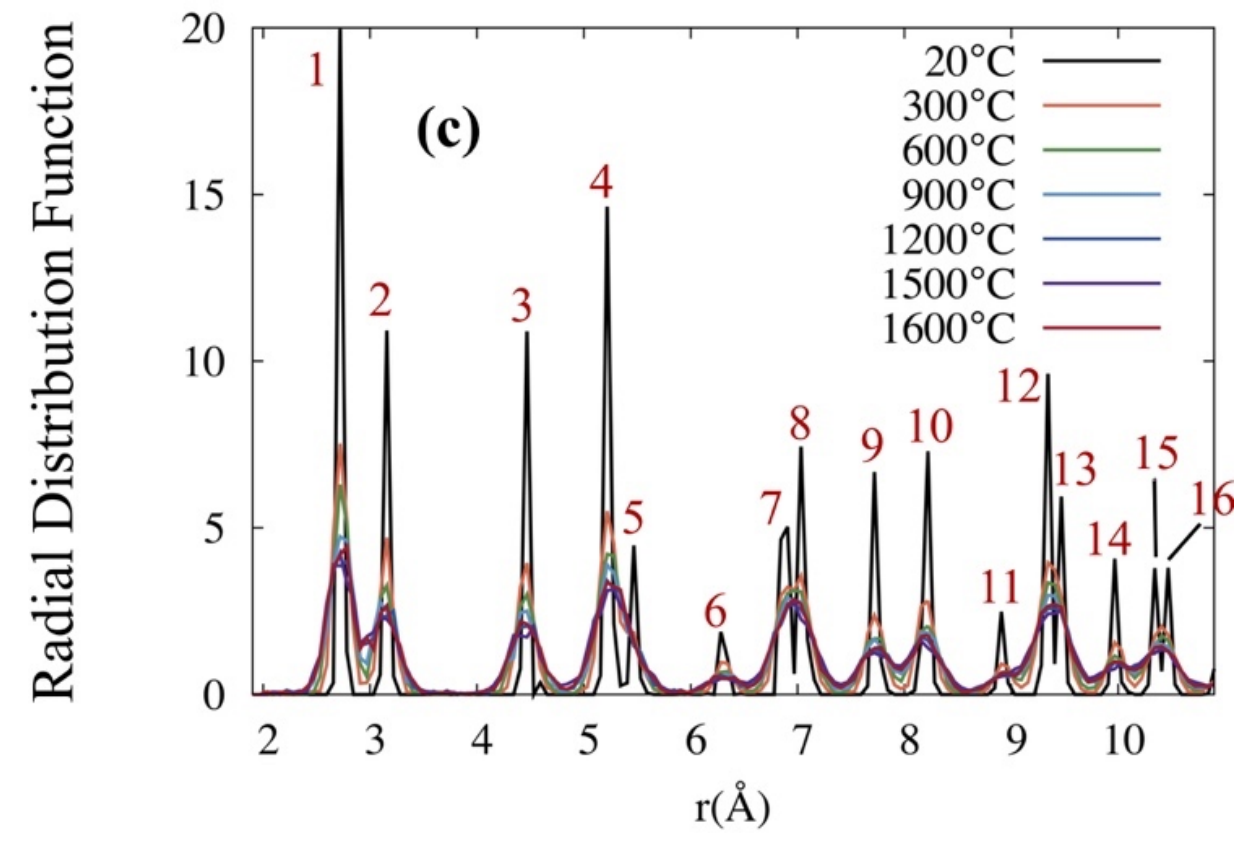

\section{Figure 2.5 Example of using the Radial Distribution Function for the HTIR-Mo system as it is heated from 20 to $1600{ }^{\circ} \mathrm{C}$. The peaks at $20^{\circ} \mathrm{C}$ are numbered for discussion in the text.}

To determine the activation energy for an adsorbate to get into a surface, the Nudged Elastic Band (NEB) method can be used [2]. This method considers an initial and a final position for a mechanism of interest and creates a number of equidistant "images" or intermediary atomic positions between the two. Each image is run through a full electronic SCF to determine the total energy as the mechanism progresses. Using the climbing image improvement to NEB, one of the images is pushed up the energy gradient to the saddle point, ensuring the transition state energy is determined [7]. Figure 2.6(a) 
shows an example transition that can be studied using NEB consisting of an $\mathrm{O}$ atom at an adsorbed hollow site moving to an interstitial site in a Mo surface. Figure 2.6(b) shows the corresponding NEB energy profile with 6 images created between the initial and final positions. Image 4 gives the saddle point energy as $3.08 \mathrm{eV}$.

(a)
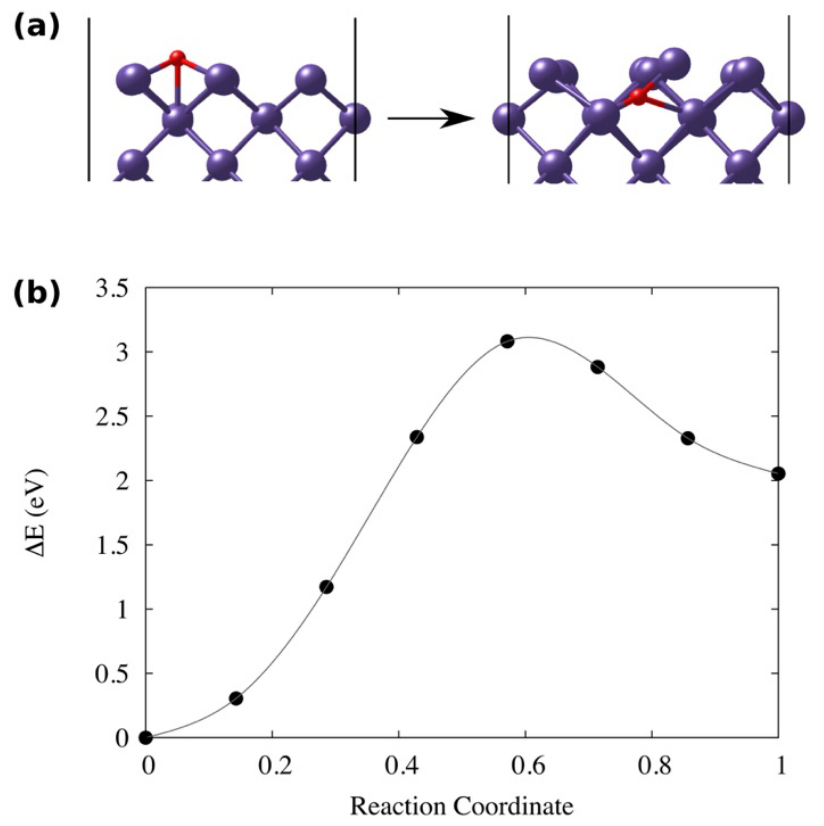

Figure 2.6 Example of using Nudged Elastic Band (NEB) to find the transition energy of (a) an $O$ atom moving from an adsorbed hollow site to an interstitial site in Mo. (b) Energies for the initial and final position of the $O$ atom as well as 6 intermediary images.

In AIMD simulations, atomic movement allows for calculation of the diffusion coefficient, $D$, using the mean squared displacement of the atoms [1]:

$$
D=\frac{\langle\vec{r}(t)-\vec{r}(0) \mid\rangle}{6 t} \quad . \quad \text { Eq. 2-13 }
$$

If the diffusion coefficient is evaluated at a range of temperatures, then the activation energy, $E_{a}$, and pre-exponential factor, $D_{0}$, can be found from the Arrhenius fit [8]: 


$$
D=D_{0} \exp \left(\frac{-E_{A}}{k_{B} T}\right) \quad \text { Eq. }(2-14)
$$

An example of diffusion coefficients is given in Figure 2.7. By comparing the trendlines, the resistance or susceptibility of HTIR thermoelements to oxidation can be compared. In comparison to the NEB method, calculating the diffusion coefficient is especially useful for finding the energy barriers to mechanisms that include multiple atoms.

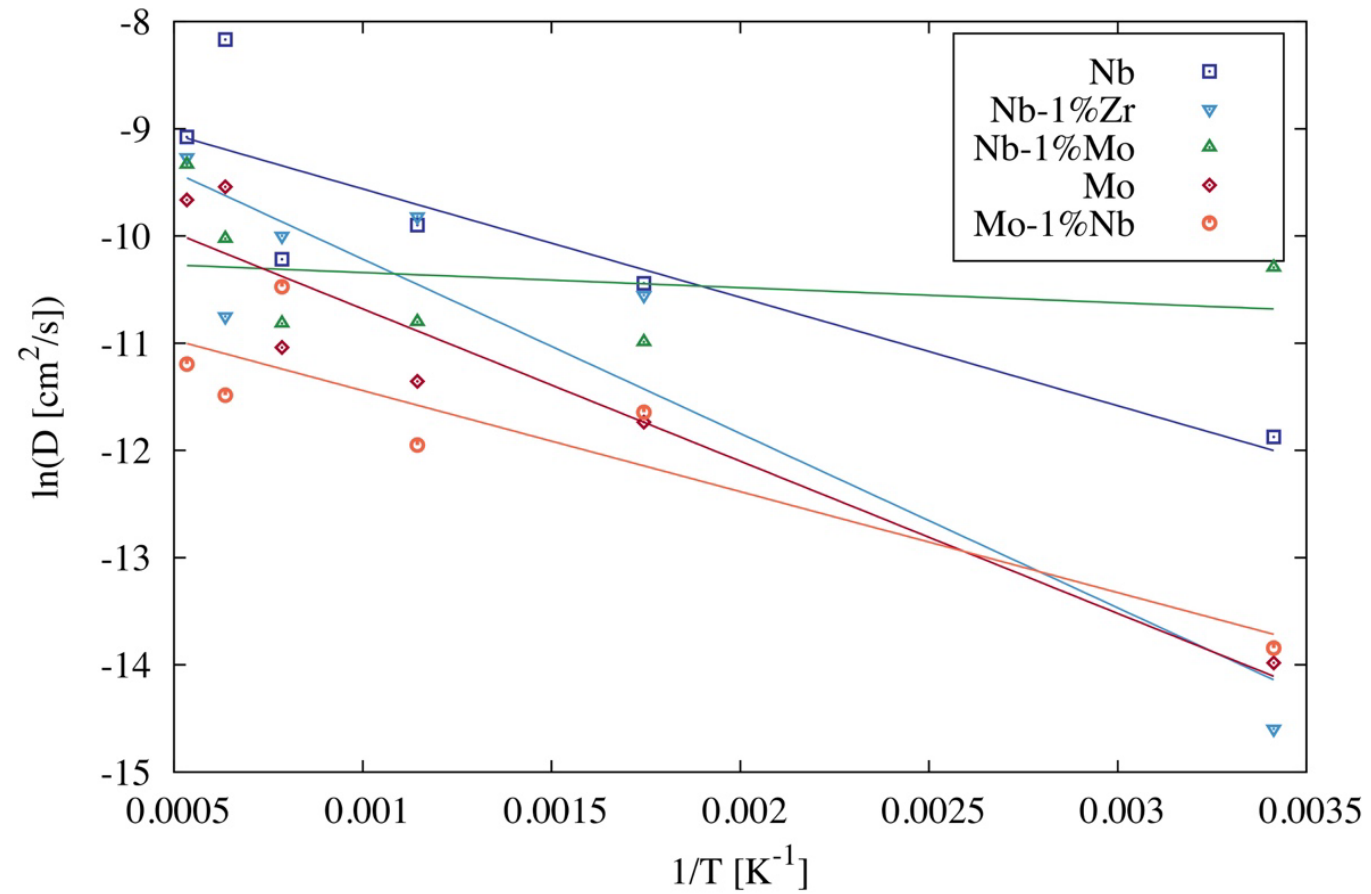

Figure 2.7 O diffusion coefficients for HTIR thermoelements exposed to $\mathrm{O}_{2}$. 


\section{References}

[1] J.G. Lee, Computational Materials Science: An Introduction, CRC Press, 2012.

[2] D.S. Sholl, J.A. Steckel, Density Functional Theory: A Practical Introduction, 2009.

[3] D. Gryaznov, E. Heifets, E. Kotomin, The first-principles treatment of the electron-correlation and spin-orbital effects in uranium mononitride nuclear fuels, Phys. Chem. Chem. Phys. 14 (2012) 4482-4490. doi:10.1039/c2cp40297a.

[4] S.L. Dudarev, G.A. Botton, S.Y. Savrasov, Z. Szotek, W.M. Temmerman, A.P. Sutton, Electronic Structure and Elastic Properties of Strongly Correlated Metal Oxides from First Principles: LSDA + U, SIC-LSDA and EELS Study of UO2 and NiO, Phys. Status Solidi. 166 (1998) 429-443. doi:10.1002/(SICI)1521396X(199803)166:1<429::AID-PSSA429>3.0.CO;2-F.

[5] P. Fuentealba, E. Chamorro, J.C. Santos, Chapter 5: Understanding and using the electron localization function, in: Theor. Asp. Chem. React., Elsevier, 2007: pp. 57-85. doi:10.1016/S1380-7323(07)80006-9.

[6] V. Polo, J. Andres, S. Berski, L.R. Domingo, B. Silvi, Understanding Reaction Mechanisms in Organic Chemistry from Catastrophe Theory Applied to the Electron Localization Function Topology, J. Phys. Chem. A. 112 (2008) 71287136. doi:10.1021/jp801429m.

[7] G. Henkelman, B.P. Uberuaga, H. Jónsson, A climbing image nudged elastic band method for finding saddle points and minimum energy paths, J. Chem. Phys. 113 (2000) 9901. doi:10.1063/1.1329672.

[8] X. Zhao, H. Jin, Investigation of hydrogen diffusion in supercritical water: A molecular dynamics simulation study, Int. J. Heat Mass Transf. 133 (2019) 718 728. doi:10.1016/j.ijheatmasstransfer.2018.12.164. 


\section{CHAPTER THREE: FIRST-PRINCIPLES COMPARATIVE STUDY OF UN AND ZR CORROSION}

This chapter is published by Elsevier in Journal of Nuclear Materials and should be referenced appropriately.

Reference:

E.L. Sikorski, T.H. da Silva, L.K. Aagesen, B.J. Jaques, L. Li, First-principles

comparative study of UN and Zr corrosion, J. Nucl. Mater. 523 (2019) 402-412. doi:10.1016/j.jnucmat.2019.06.017.

Reproduced from J. Nucl. Mater. 523 (2019), with the permission of Elsevier.

*This chaper includes modifications from the originally published version. 
First-principles comparative study of $\mathrm{UN}$ and $\mathrm{Zr}$ corrosion

\author{
Ember L. Sikorski ${ }^{a, b}$ \\ Thiago H. da Silva ${ }^{a}$ \\ Larry K. Aagesen ${ }^{\mathrm{c}}$ \\ Brian J. Jaques ${ }^{\mathrm{a}, \mathrm{b}}$ \\ Lan $\mathrm{Li}^{\mathrm{a}}$
}

Published in:

Journal of Nuclear Materials

June 2019

${ }^{a}$ Micron School of Materials Science and Engineering, Boise State University, Boise, ID, 83725, USA

${ }^{b}$ Center for Advanced Energy Studies, Idaho Falls, ID, 83401, USA

${ }^{c}$ Fuels Modeling and Simulation Department, Idaho National Laboratory, Idaho Falls, ID, 83415, USA 


\begin{abstract}
We studied surface corrosion effects on $\mathrm{Zr}$ and UN using first-principles density functional theory-based calculations. We focused on the energetics of $\operatorname{Zr}$ (1000), UN (100) and UN (110) surfaces, exposed to water and oxygen. Average distance between the terminating UN (100) surface and bulk increases due to the presence of additional oxygen content, as well as for the (110) surface. The average distance between the surface layer and bulk is greater in the (110) surface than the (100) surface after water adsorption. Oxygen concentration determines whether $\mathrm{H}_{2}$ or oxynitride is formed on the (110) surface. Local density of states and partial charge density show the bonding between the UN surfaces and adsorbates. From an electronic energy of $-2 \mathrm{eV}$ to the Fermi level, the majority of electrons are found to be localized around $U$ atoms. Electron localization function calculations further reveal the corrosion mechanism details.
\end{abstract}

\title{
3.1 Introduction
}

The higher thermal conductivity, higher fissile density, and easier reprocessability of uranium nitride (UN) in comparison to uranium dioxide $\left(\mathrm{UO}_{2}\right)$ have driven its development for Generation IV reactors [1-4]. While UN offers many benefits as a prospective fuel, it corrodes when exposed to oxygen and water, losing much of its structural integrity. In the event of cladding failure, UN pellets would inevitably degrade, leading to fuel release [2]. With a better understanding of this corrosion process, materials strategies can be developed to improve the stability of UN.

Many studies have probed the UN corrosion mechanism, beginning with Dell et al. [5] who proposed:

$$
\mathrm{UN}+2 \mathrm{H}_{2} \mathrm{O} \rightarrow \mathrm{UO}_{2}+\mathrm{NH}_{3}+\frac{1}{2} \mathrm{H}_{2} \quad \text {. } \quad \text { Eq. (3-1) }
$$


However, even from this first study they noted not all of the compounds followed the reaction to completion, with some specimens yielding $\mathrm{U} 2 \mathrm{~N} 3+\mathrm{x}$ and residual N. Shortly after, Sugihara et al. [6] proposed the formation of an intermediary phase of the form $\mathrm{UO}_{\mathrm{x}} \mathrm{N}_{\mathrm{y}}$. This oxynitride phase has continually been identified in subsequent studies $[3,4,7-10]$, though rarely with the same stoichiometry. In fact, much of the UN corrosion field yields little consensus. For instance, Liu et al. [7] proposed a mechanism leading from the oxynitride phase to $\mathrm{UO}_{2}$ as:

$$
2 \mathrm{UO}_{2} \mathrm{~N}_{x}+x \mathrm{O}_{2} \rightarrow 2 \mathrm{UO}_{2}+2 x \mathrm{NO} \quad \text {. } \quad \text { Eq. (3-2) }
$$

In contrast, Jolkkonen et al. [2] detected no NO production during hydrolysis. Such discrepancies are likely the result of differences in starting materials and experimental setups. Recently, $\mathrm{Lu}$ et al. [8] showed whether $\mathrm{UO}_{2}$ or $\mathrm{UO}_{\mathrm{x}} \mathrm{N}_{\mathrm{y}}$ formed depended on the amount of $\mathrm{N}$ content in their $\mathrm{UN}_{\mathrm{x}}$ films.

With the variation of both reactants and products across UN corrosion experiments, Density Functional Theory (DFT) studies offer the capability to start with pure UN and add precisely the desired reactants. Bocharov et al. [3] started with UN surfaces (100) and (110) and a tilt GB and added oxygen adsorbates. Oxygen spontaneously adsorbed on both the surfaces and the GB, but energetically favored the surfaces. Nitrogen vacancies were also considered, and while the nitrogen vacancy formation energy was higher on the (100) surface than the (110) surface, oxygen preferred the (100) $\mathrm{N}$ vacancy. Li et al. [11] conducted a similar surface study of single atom adsorption and using Projected Density of States (PDOS) found electron transfer from $\mathrm{U}$ to $\mathrm{O}$ on the $\mathrm{UN}$ (111) surface after $\mathrm{O}$ chemisorption. Oxygen equally favored hollow and bridge sites over U-top sites on the (111) surface. Bo et al. included both 
partially dissociative and fully dissociative water, from one molecule up to 1 monolayer (ML) coverage on the UN (100)[1] and (110)[12] surfaces. Furthermore, ab-initio atomistic thermodynamics showed water adsorption on $\mathrm{UN}$ surfaces readily occurred at room temperature [1]. Still, computational studies have not yet determined a UN corrosion mechanism.

To better understand the UN corrosion mechanism, we consider the nuclear cladding component $\mathrm{Zr}$, for which the corrosion mechanism is known:

$$
\mathrm{Zr}+2 \mathrm{H}_{2} \mathrm{O} \rightarrow \mathrm{ZrO}_{2}+2 \mathrm{H}_{2} \quad \text {. } \quad \text { Eq. (3-3) }
$$

For this system, the mechanism is heavily driven by the electronic structure [13,14]. $\mathrm{Zr}$ oxide growth is governed by the conductivity of the film, with corrosion increasing as electrical conductivity increases [13]. The oxygen concentration gradient drives oxygen anions through the film to form new oxide at the oxide-zirconium interface [14]. This new oxide then releases electrons that migrate to the hydrogen ions. The electric potential also drives the charged hydrogen through the oxide to the metal, resulting in hydrogen pickup. Low-energy electron diffraction (LEED) has shown that at $0.5 \mathrm{ML}$ O coverage, O atoms occupy the octahedral sites between the first, second, and third layers of $\operatorname{Zr}$ [15]. At $2 \mathrm{ML}, \mathrm{O}$ atoms occupy surface face centered cubic (SFCC) sites and tetrahedral sites between the first and second layers. Several DFT studies have examined the behavior of $\mathrm{O}$ on the $\mathrm{Zr}$ surface. Wang et al. [16] and Jomard et al. [17] showed favorable adsorption at the SFCC site. Chiang et al. [18] suggested the lowest energy barrier pathways for O

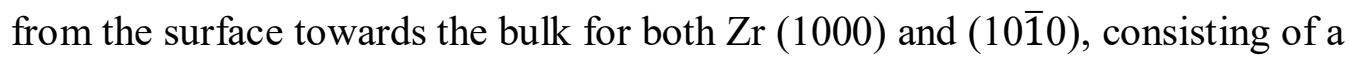
combination of octahedral and tetrahedral interstitial sites. To our knowledge, DFT 
studies have not previously mapped the electronic structure of the $\mathrm{Zr}$ surface in the presence of $\mathrm{O}$, though.

Following the dependence of $\mathrm{Zr}$ corrosion on the electronic profile, this paper presents the detailed electronic calculations of both $\mathrm{Zr}$ and $\mathrm{UN}$ corrosion mechanisms for comparison. We calculated the electronic structure of a $\mathrm{Zr}$ surface in the presence of water and oxygen, followed by the UN surfaces. The following sections are organized as follows: in Section 3.2 we describe the computational methods used on both the UN and $\mathrm{Zr}$ systems. In Section 3.3.1, we examine our electronic structure results for $\mathrm{Zr}$ through the lens of the experimental literature. In Section 3.3.2 we begin by examining the accuracy of our UN calculations and then compare our electronic structure calculations to the Zr system results. In Section 3.4, we compare our results with previous UN corrosion studies and discuss the differences between the $\mathrm{Zr}$ and $\mathrm{UN}$ system electronic profiles. Finally, in Section 3.5 we summarize our key findings and UN corrosion mechanism hypotheses from this study.

\subsection{Methods}

The Vienna ab initio Software Package (VASP) was used to perform density functional theory (DFT) calculations [19]. Spin-polarized generalized gradient (GGA) exchange-correlation functions were used within the Perdew Burke Ernzerhof (PBE) [20] formalism. To account for Fermi surface broadening, a Gaussian smearing value of 0.05 was utilized. Plane-wave basis sets were implemented utilizing the projector-augmented wave (PAW) pseudopotentials to a mesh cutoff energy of $500 \mathrm{eV}$. Due to the localized nature of the f-shell electrons of uranium, the Hubbard-U GGA + U approach of Dudarev et al. [21] was implemented on all UN systems. The Coulomb potential (U) was set to 
$2.40 \mathrm{eV}$ and exchange energy ( $\mathrm{J})$ to $0.50 \mathrm{eV}$ (i.e. $\mathrm{U}_{\text {eff }}=1.90 \mathrm{eV}$ ), as previously chosen by Bo et al. [1,12] due to the success of two independent studies. Firstly, Lu et al. [22] demonstrated $\mathrm{U}_{\text {eff }}=2.00 \mathrm{eV}$ reproduced the lattice parameter, bulk modulus, and phonon properties in good agreement with experiment. Secondly, Gryaznov et al. [3] showed $\mathrm{U}_{\text {eff }}=1.85 \mathrm{eV}$ reproduced both the antiferromagnetic alignment as the most stable state for bulk $\mathrm{UN}$ as well as a comparable magnetic moment to experiment. Thus, $\mathrm{U}_{\mathrm{eff}}=1.90 \mathrm{eV}$ was selected in our calculations to balance the success of $\mathrm{Lu}$ et al. and Gryaznov et al. The agreement of this effective Hubbard parameter is further demonstrated in Section 3.3.2. All structures were relaxed to a final residual force break condition of $0.01 \mathrm{ev} \AA^{-1}$. 4-, 6- and 8-monolayer- slabs were tested for convergence. We found that the surface energy was converged with the 4-monolayer slab with a difference of less than $0.01 \mathrm{ev} \AA^{-}$ ${ }^{2}$ between slabs. To verify that the choice of four monolayers had no significant effect on adsorption mechanism, we compared the binding energies for $\mathrm{O}$ on different (100) slabs using [3]:

$$
\Delta E_{\text {bind }}^{O}=\frac{1}{2}\left(E^{U N}-E_{O_{a d s}}^{U N}+E^{O}\right) \quad, \quad \text { Eq. }(3-4)
$$

where $E^{U N}$ is the energy of the pure UN slab, $E_{O_{a d s}}^{U N}$ is the energy of the slab with adsorbed $\mathrm{O}$, and $E^{O}$ is the energy of a single $\mathrm{O}$ atom. The binding energies are shown in Table 3.1. The larger the binding energy, the more stable the adsorption. O energetically prefers the $\mathrm{U}$ top site than the $\mathrm{N}$ top site, and both the 4- and 8-monolayer slabs show the same change trend for the $\mathrm{O}$ binding energy. To further explore the effect of $\mathrm{O}$ adsorption, the respective Partial Charge Density (PCD) and Electron Localization Function (ELF) were calculated, as shown in Figure 3.1. The PCD shows a map of the valence electrons in a specific energy range, in this case from $-2 \mathrm{eV}$ to the Fermi Level. 
On the other hand, the ELF maps all the calculated electrons. In each case, while the third and fourth layers from the surface are slightly different, the charge profile at the surface of the 4-monolayer and 8-monolayer slabs are identical. The bond lengths between $\mathrm{O}$ and the respective surface $\mathrm{U}$ or $\mathrm{N}$ atom for the 4- and 8-monolayer slabs are converged to $0.01 \AA$. For these reasons, the 4-monolayer slab was selected for computational efficiency. A gamma-centered $2 \times 2 \times 1$ k-point mesh was used for geometry optimizations after convergence testing. A denser $4 \times 4 \times 1$ k-point mesh was used for the electronic structure calculations.

Table 3.1 Calculated binding energies for $\mathrm{O}$ at $\mathrm{U}$-top and $\mathrm{N}$-top sites on 4-, 6and 8-monolayer slabs.

\begin{tabular}{lll}
\hline Monolayers & U-top $(\mathrm{eV}$ per O) & N-top $(\mathrm{eV}$ per O) \\
\hline 4 & 4.79 & 3.84 \\
6 & 4.17 & 3.57 \\
8 & 4.36 & 4.30 \\
\hline
\end{tabular}




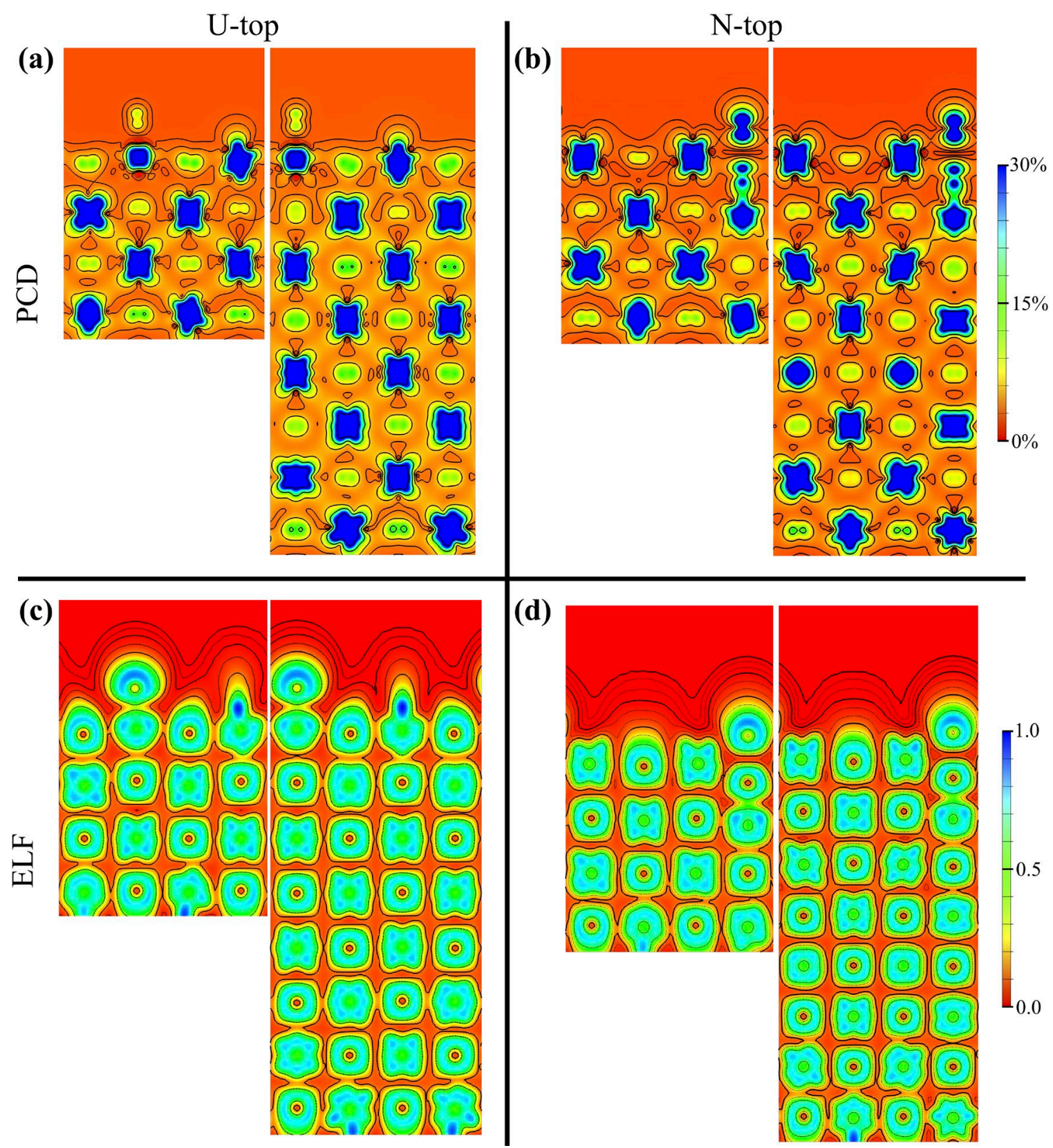

Figure 3.1 Comparison of the Partial Charge Density (PCD) (a-b) and Electron Localization Function (ELF) (c-d) for O adsorption on an 4-monolayer slab (left) and 8-monolayer slab (right). The $O$ was placed on top of different $U$ atoms between the 4- and 8-monolayer slab, resulting in a lateral shift of the charge maps for (a) and (c). Contour lines indicate areas with the same energy. In the ELF, 0 represents low probability of finding an electron and 1 represents high probability of finding an electron. The PCD shows the density of states from $-2 \mathrm{eV}$ to the Fermi level mapped onto the structure. The PCD map has been narrowed to $30 \%$ of the total states to better visualize the bonds. 
UN (100) (Figure 3.2(a \& b)) and (110) (Figure $3.2(\mathrm{~d} \& \mathrm{e})$ ) surfaces were created with $4 \times 4 \times 1$ and $4 \times 2 \times 1$ supercells, respectively. The positions of the atoms in the bottom two monolayers were fixed to simulate the bulk. The top two layers were allowed to move to depict the reconfiguration of the surface in the presence of water and oxygen.

To select initial adsorbate configurations, we considered the previous UN adsorption studies. Li et al. reported single $\mathrm{O}$ atom adsorption at the U-top, N-top, hollow, and bridge sites on UN (100) and (111) [11]. Li et al. found the hollow site to be the most stable on the (100) surface, and the hollow and bridge site to be equally stable on the (111) surface. Bocharov et al. reported $\mathrm{O}$ atom binding energies at $\mathrm{U}$-top and $\mathrm{N}$ top sites on the (100) and (110) surfaces, with the U-top position being the most stable on each surface [3]. Bo et al. explored water adsorption up to 4 molecules on the (100) and (110) surfaces $[1,12]$. For single molecule adsorption on (100), stable configurations occurred at hollow and U-top sites. For $\mathrm{H}, \mathrm{O}$, and $\mathrm{OH}$ on the (110) surface, the so-called uranium-bridge site was the most stable. We called this site a U-top site as the adsorbate lies over a $\mathrm{U}$ atom in the second layer. Due to the variations in calculation parameters across these studies, we performed adsorption calculations of a single $\mathrm{O}$ atom at $\mathrm{U}$-top, N-top, bridge, and hollow sites on UN (100). U-top and hollow sites give adsorption energies of -12.87 and $-7.48 \mathrm{eV}$, respectively. However, the $\mathrm{O}$ placed at the $\mathrm{N}$-top site relaxed to the hollow position, and the O placed at the bridge site relaxed to the U-top position. Due to the findings of Li et al. reporting bridge and hollow sites as energetically equivalent and Bo et al. finding no stable adsorption at the bridge site, we did not consider this site for any adsorbates. Conversely, due to the prevalence of N-top placement across studies, we included the N-top site. 
The chosen adsorption sites were U-top and hollow sites, due to their favorability, and N-top due to its prevalence across studies. The adsorbates were placed to include all three of these sites, as shown in Figure 3.2(g) and Figure 3.3(a-b). While it is possible for adsorbates to relax to a local minima, each was placed at least $2 \AA$ away from the UN surface to reduce the possibility of becoming confined to a high symmetry local minima. Half of the $\mathrm{OH}$ species were positioned with $\mathrm{O}$ closest to the metal surface and half with $\mathrm{H}$ closest to the metal surface. We chose 8 species to represent full saturation (1 monolayer) of the UN (100) surface, defined by 1 water molecule to 1 metal surface atom. Due to the dense population of the dissociated water adsorbents, the additional 4 oxygen atoms were added $1 \AA$ above the dissociated water layer (Figure 3.2 (d-f) and Figure 3.3(c-d)). The vacuum was set to approximately $30 \AA$ to minimize interactions between subsequent slabs. The Zr (1000) surface (Figure 3.2 (c \& f)) was created with 4 monolayers and a surface of $2 \times 2$ atoms. The bottom 2 layers were fixed and the top 2 layers were allowed to relax. Eight dissociated water species were placed at a minimum 2 $\AA$ distance from each surface (Figure 3.2 (c)) and a second set of calculations added 4 oxygen atoms $1 \AA$ above the dissociated water layer (Figure 3.2 (f)). The vacuum was set to approximately $30 \AA$.

Local Density of States (LDOS) calculations gave a graphical representation of the electrons localized to each element with respect to energy, as well as revealed if bonding was covalent or ionic through overlap or lack thereof, respectively. PCD and ELF [23] calculations were then performed to further examine the electronic structure and evolution of the surface as oxygen concentration increased. PCD mapped the density of states onto the atomic structure. The PCD resolved specific energy ranges [22,24], 
such as valence electrons near the Fermi level (by convention set to $0 \mathrm{eV}$ ). We used PCD to examine the energy ranges of $-5 \mathrm{eV}$ to the Fermi level and $-2 \mathrm{eV}$ to the Fermi level. The ELF was used to consider all calculated electrons, where core electrons were treated as part of the nucleus. While the PCD is a map of the DOS, the ELF is its own calculation based on the orbitals. The ELF is based on the Pauli exclusion principle and calculates the probability of finding another electron in the vicinity of a reference electron with the same spin [25]. Noting that the lower the probability of finding a nearby same spin electron is, the more localized the reference electron is, the ELF converts this probability to a measure of localization. In this function, 0 represents low probability of finding a localized electron, $1 / 2$ represents a probability equivalent to a homogeneous electron gas, and 1 represents high probability of finding a localized electron. The ELF gives a more complete description of the electronic profile, as opposed to that of a specific energy range in the PCD. 


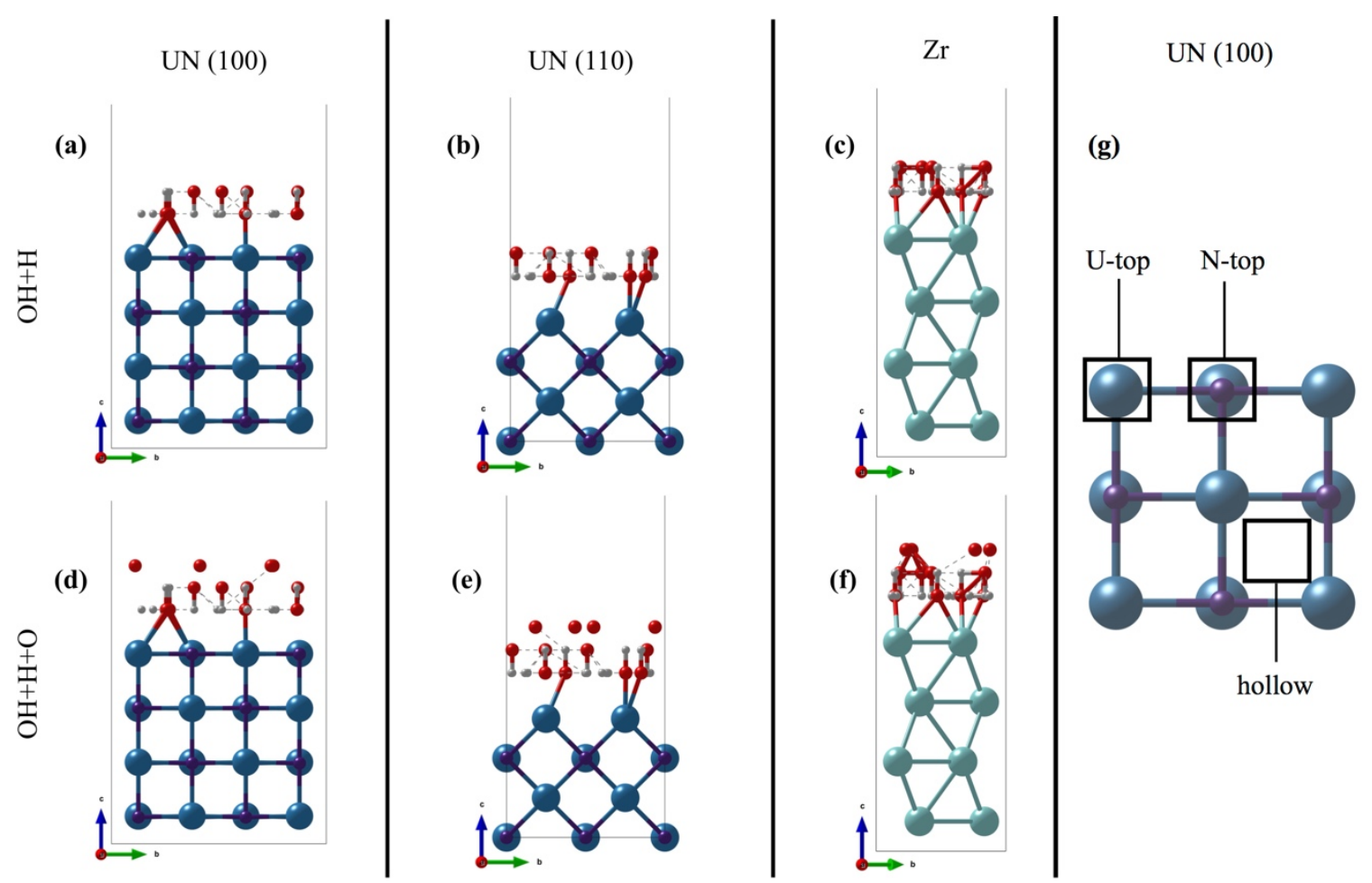

Figure 3.2 Initial configurations: $\mathrm{UN}$ and $\mathrm{Zr}$ surfaces with $\mathrm{OH}+\mathrm{H}(\mathrm{a}-\mathrm{c})$ and $\mathrm{OH}+\mathrm{H}+\mathrm{O}(\mathrm{d}-\mathrm{f})$. Top-down view of adsorption sites on $\mathrm{UN}(100)(\mathrm{g}) . \mathrm{U}, \mathrm{N}, \mathrm{Zr}, \mathrm{O}$, and $H$ are represented by blue, purple, teal, red, and gray, respectively.

$(100)$

$\mathrm{OH}+\mathrm{H}$

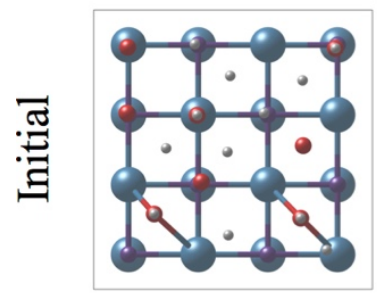

(a)

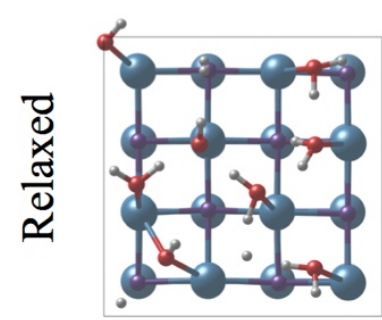

(e)

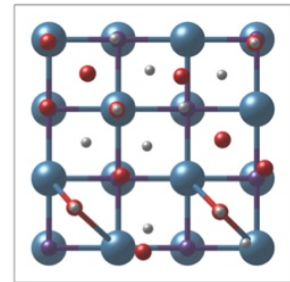

(b)

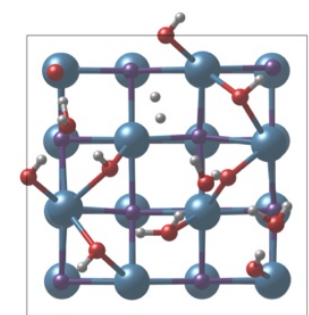

(f)
$\mathrm{OH}+\mathrm{H}$

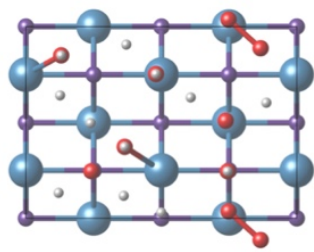

(c)

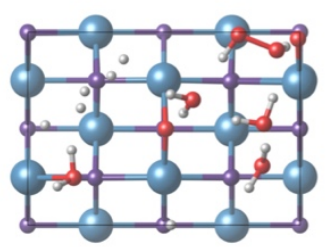

(g)
$\mathrm{OH}+\mathrm{H}+\mathrm{O}$

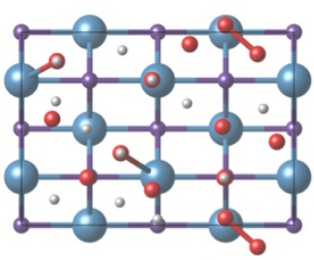

(d)

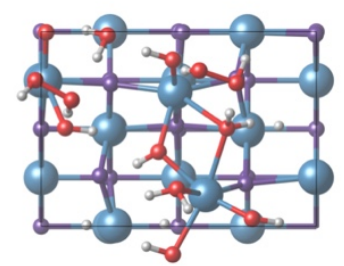

(h)

Figure 3.3 Top-down view comparison between initial configurations of $U N$ surfaces (a-d) and relaxed configurations of $U N$ surfaces (e-h). $U, N, O$, and $H$ are represented by blue, purple, red, and gray, respectively. 


\subsection{Results}

\subsubsection{Zirconium}

After starting with dissociated water on the $\mathrm{Zr}$ surface (Figure 3.2(c)) and allowing the system to relax (Figure $3.5(\mathrm{c})$ ), $12.5 \%$ of oxygen penetrates the surface and bonds to a terminating $\mathrm{Zr}$ and a second layer $\mathrm{Zr}$ at bond lengths of 2.03 and $2.16 \AA$, respectively. $12.5 \% \mathrm{O}$ coordinates with three surface $\mathrm{Zr}$ with $\mathrm{Zr}$-O bond lengths from $2.16-2.18 \AA .12 .5 \%$ of $\mathrm{O}$ forms $\mathrm{OH}$ and coordinates with two $\mathrm{Zr}$ at bond lengths of $\mathrm{Zr}$ $\mathrm{O}$ (h) 2.30 and $2.32 \AA .12 .5 \%$ forms water and coordinates with a surface $\mathrm{Zr}$ at a $\mathrm{Zr}-\mathrm{O}(\mathrm{w})$ distance of $2.56 \AA$. The remaining $\mathrm{O}$ forms $\mathrm{H}_{2} \mathrm{O}$ and does not adsorb to the surface, while $12.5 \% \mathrm{H}$ forms $\mathrm{H}_{2}$ and the rest do not form bonds. The water adsorption results in a terminating layer separation increase of $7.2 \%$.

With additional $\mathrm{O}$, the system relaxes from (Figure 3.2 (f)) to (Figure 3.3 (f)). $8 \%$ of $\mathrm{O}$ also penetrates the surface and coordinates with a terminating and second layer $\mathrm{Zr}$ at bond lengths of 2.09 and $2.18 \AA .8 \%$ of $\mathrm{O}$ coordinates with three $\mathrm{Zr}$ with $\mathrm{Zr}-\mathrm{O}$ distances ranging from $2.04-2.11 \AA .16 \%$ forms $\mathrm{OH}$ and coordinates with two $\mathrm{Zr}$ at $\mathrm{Zr}-\mathrm{O}$ (h) bond lengths of 2.28/2.34 and 2.29/2.35 $\AA$. $8 \%$ of $\mathrm{OH}$ bonds to $\mathrm{ar}$ at $2.04 \AA$ and pulls the $\mathrm{Zr}$ away from the surface, and giving an average terminating layer separation increase of $16.9 \%$.

The overlap in the LDOS (Figure 3.4) shows covalent bonds between the dissociated water and the $\mathrm{Zr}$ surface, notably from -10 to $-4 \mathrm{eV}$. Due to the primarily ionic nature of the bonds from $-5 \mathrm{eV}$ to the Fermi level, the PCD maps for this range (not pictured) give minimal information on the electronic structure of the whole system. Unlike PCD, the ELF can show the most probable locations of finding any calculated 
electron, rather than only valence electrons. Using the ELF, we examined the nature of the charge transfer and compared it with the well-known $\mathrm{Zr}$ corrosion mechanism [14]:

$$
\begin{aligned}
& \text { (1) } 2 \mathrm{H}_{2} \mathrm{O} \rightarrow 2 \mathrm{O}^{2-}+4 \mathrm{H}^{+} \\
& \text {(2) } \mathrm{O}^{2-}+\mathrm{V}_{\mathrm{O}} \rightarrow \mathrm{O}_{\text {adsorbed }}^{2-} \\
& \text { (3) } \mathrm{Zr} \rightarrow \mathrm{Zr}^{4+}+4 e^{-} \\
& \text {(4) } \mathrm{Zr}^{4+}+2 \mathrm{O}^{2-} \rightarrow \mathrm{ZrO}_{2} \\
& \text { (5) } 4 \mathrm{H}^{+}+4 e^{-} \rightarrow 2 \mathrm{H}_{2}
\end{aligned}
$$

As shown in Figure 3.5 (c) and Figure 3.4 (f), though the ions started as dissociated water in the form of $\mathrm{OH}$ and $\mathrm{H}$ ions, many revert to the molecular structures. In this manner, the ions spontaneously form their optimal phase (molecular, partially dissociative, or fully dissociated) regardless of the starting phase. Though many of the dissociated oxygen ions form an $\mathrm{OH}$ ion or $\mathrm{H}_{2} \mathrm{O}$ molecule, $12.5 \%$ of the $2 \mathrm{ML} \mathrm{O}$ penetrates the surface, depicting steps (1) and (2) of the Zr corrosion mechanism. In step (3) $\mathrm{Zr}$ gives off electrons to form a positive surface. In Figure 3.6(a), the $\mathrm{Zr}$ displays a yellow and orange terminating region indicating increased positive charge and the center of the terminating orbitals have less blue character or electrons than the second layer orbitals. In Figure 3.6(b) and (c), each terminating $\mathrm{Zr}$ bonds to at least two O, indicating the formation of $\mathrm{ZrO}_{2}$ as part of step (4). Finally, both Figure 3.6(b) and (c) display more electrons localized at the $\mathrm{H}$ sites than any other region of the electron localization map, as expected from step (5). 

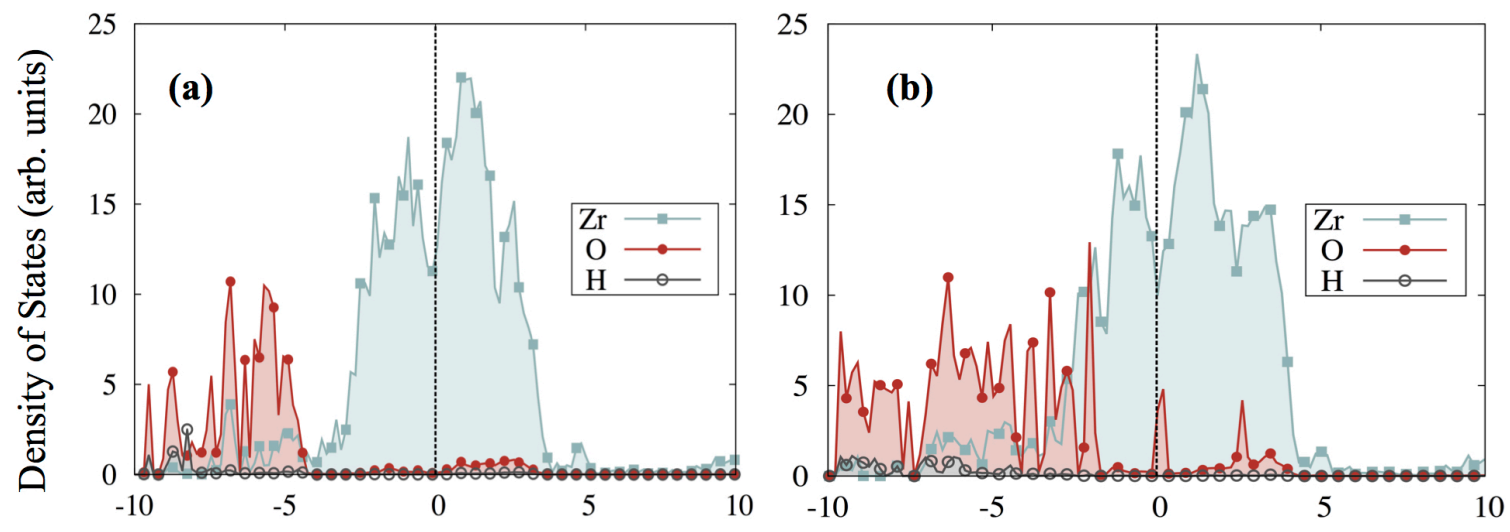

Energy (eV)

Figure 3.4 Local density of states of (a) $\mathrm{Zr}$ (1000) surface with $\mathrm{OH}+\mathrm{H}$ and (b) with $\mathbf{O H}+\mathrm{H}+\mathbf{O}$.
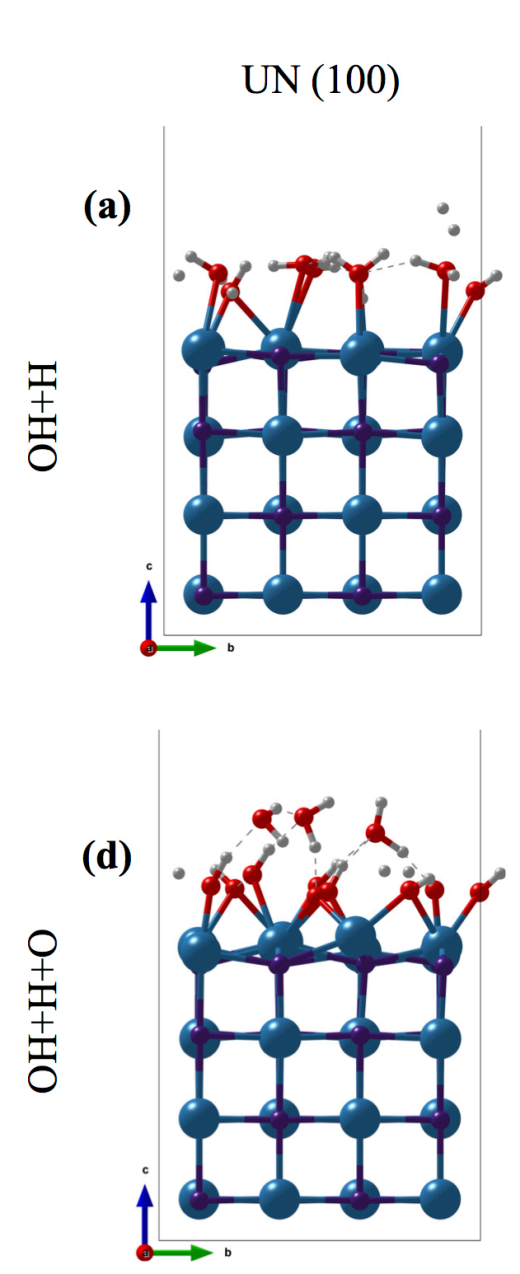

(b)

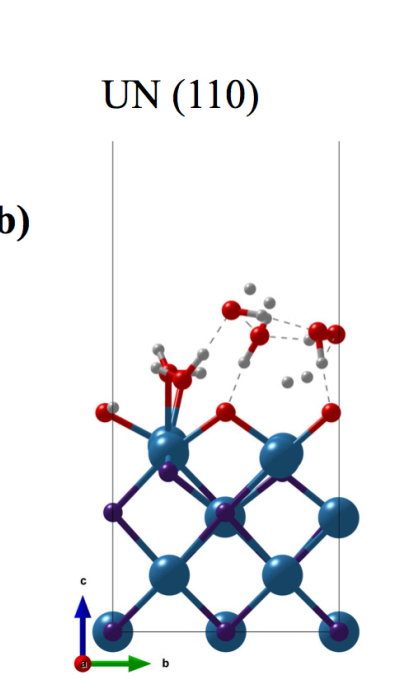

(e)

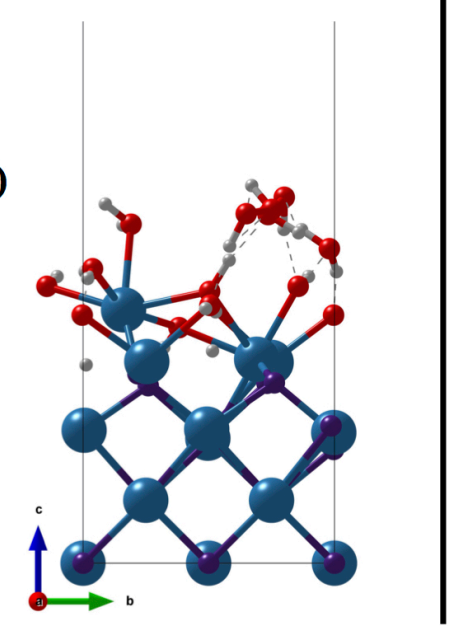

(c)

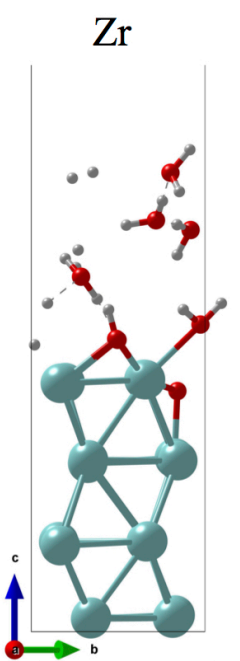

(f)

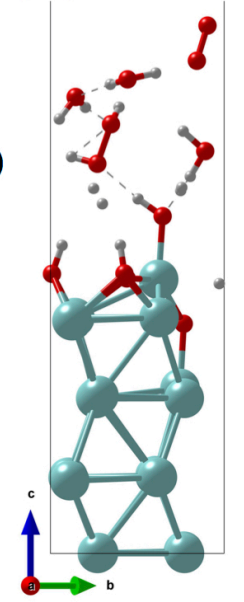

Figure 3.5 Relaxed configurations: $\mathrm{UN}$ and $\mathrm{Zr}$ surfaces with $\mathrm{OH}+\mathrm{H}(\mathrm{a}-\mathrm{c})$ and $\mathrm{OH}+\mathrm{H}+\mathrm{O}(\mathrm{d}-\mathrm{f}) . \mathrm{U}, \mathrm{N}, \mathrm{Zr}, \mathrm{O}$, and $\mathrm{H}$ are represented by blue, purple, teal, red, and gray, respectively. 
(a) pure $\operatorname{Zr}(1000)$
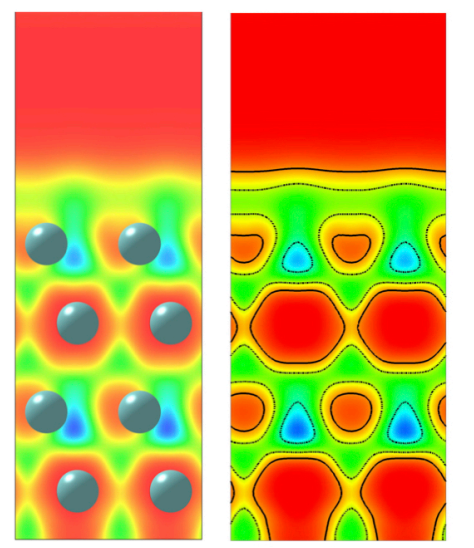

(b) $\mathrm{Zr}$ (1000) with $\mathrm{OH}+\mathrm{H}$

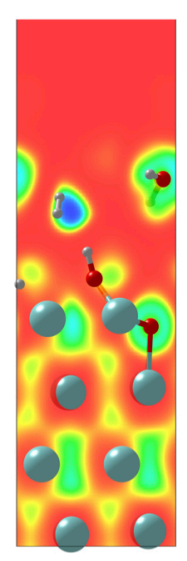

(c) $\mathrm{Zr}$ (1000) with $\mathrm{OH}+\mathrm{H}+\mathrm{O}$

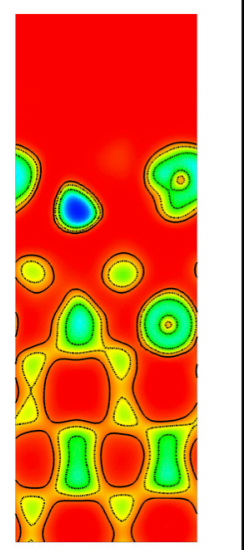

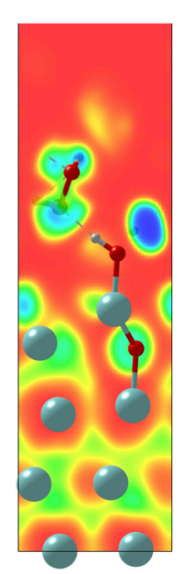

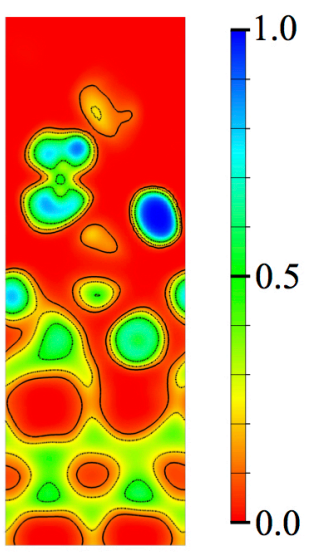

Figure 3.6 Electron Localization Function (ELF) of (a) the pure $\mathrm{Zr}$ (1000) surface, (b) with $\mathrm{OH}+\mathrm{H}$, and (c) with $\mathrm{OH}+\mathrm{H}+\mathrm{O}$. Blue and red indicate high and low probability of finding a localized electron, respectively. Contour lines indicate areas with the same energy.

\subsubsection{Uranium Nitride}

While the UN bulk exhibits type I antiferromagnetic structure [26], as previously illustrated by Claisse et al. [27], a surface is inherently less stable than the bulk due to the dangling bonds. Rafaja et al. suggested no bulk-like antiferromagnetism of UN films, replaced with ferromagnetism [28]. Therefore, we used DFT+U with ferromagnetic ordering on the UN surfaces, agreeing well with the results of Bocharov et al. $[3,10]$. Our bulk calculations yielded an average magnetic moment of $1.70 \mu \mathrm{B}$ on $\mathrm{U}$, comparable to the magnetic moments reported by Claisse et al. [27] ranging from 1.54 to $1.95 \mu \mathrm{B}$. Our surface calculations yielded an average magnetic moment of $1.33 \mu \mathrm{B}$, comparable to Bocharov et al. [29] ranging from 1.33 to $1.57 \mu \mathrm{B}$. To further benchmark our calculations, the lattice constant and bulk modulus results are compared to literature in Table 3.2 and Table 3.3, respectively. These tables confirm the ability of the $U_{\text {eff }}=1.90 \mathrm{eV}$ to yield both lattice constant and bulk modulus values in good agreement with experimental values. 
Table 3.2 Calculated UN lattice constant in comparison with experimental and theoretical data.

Experiment

[1]
This work

4.91
Bocharov et

al. [29]

Lattice constant

$(\AA)$

4.89

Bo et al. [1]

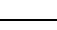

Table 3.3 Calculated UN bulk modulus (GPa) in comparison with experimental and theoretical data.

\begin{tabular}{llllll}
\hline & $\begin{array}{l}\text { Experiment } \\
{[30]}\end{array}$ & This work & $\begin{array}{l}\text { Lu et al. } \\
{[30]}\end{array}$ & $\begin{array}{l}\text { Claisse et } \\
\text { al. [27] }\end{array}$ & $\begin{array}{l}\text { Evarestov } \\
\text { et al. [31] }\end{array}$ \\
\hline $\begin{array}{l}\text { Bulk Modulus } \\
\text { (Gpa) }\end{array}$ & $\begin{array}{l}194,200, \\
206\end{array}$ & 198.5 & 194.5 & 165 & $\begin{array}{l}167.2- \\
227\end{array}$ \\
\hline
\end{tabular}

Following Tasker's analysis, (100) is the most stable UN surface [1,11,32]. Likewise, the (110) plane has the same stoichiometry and is expected to have a low surface energy [3,12]. Unlike (100) and (110), the electrostatics of (111) prohibit its formation and a complex defect structure must be formed to stabilize it. Such a structure is beyond the scope of this work. The surface energies for UN (100) and (110) were calculated using [33]:

$$
\gamma=\frac{1}{2 A}\left(E_{\text {slab }}-N E_{\text {bulk }}\right) .
$$

The results are shown in Table 3.4. The calculated surface energies generally agree well with literature. The slight difference could be the consequence of different supercell sizes considered in the calculations. To determine the nature of water adsorbates, we firstly placed molecular water and $\mathrm{O}_{2}$ on the most stable UN surface (100) at a coverage of $0.25 \mathrm{ML}$ for each species. After relaxation, $50 \%$ of $\mathrm{O}_{2}$ and $\mathrm{H}_{2} \mathrm{O}$ 
molecules remained (i.e. no chemical interaction observed) while the rest were dissociated into $\mathrm{O}, \mathrm{H}$ and $\mathrm{OH}$ ions (Figure 3.7).

Table 3.4 Surface energies from this work, Bo et al., and Bocharov et al. for a 5monolayer slab in terms of $\mathrm{eV} / \AA^{2}$. Values in parenthesis are given in $J / \mathbf{m}^{2}$.

\begin{tabular}{llll}
\hline Plane & This work & Bo et al. [12] & Bocharov et al. [3] \\
\hline$(100)$ & $0.074(1.19)$ & - & $0.090(1.44)$ \\
$(110)$ & $0.126(2.02)$ & $0.112(1.80)$ & $0.124(1.98)$ \\
\hline
\end{tabular}

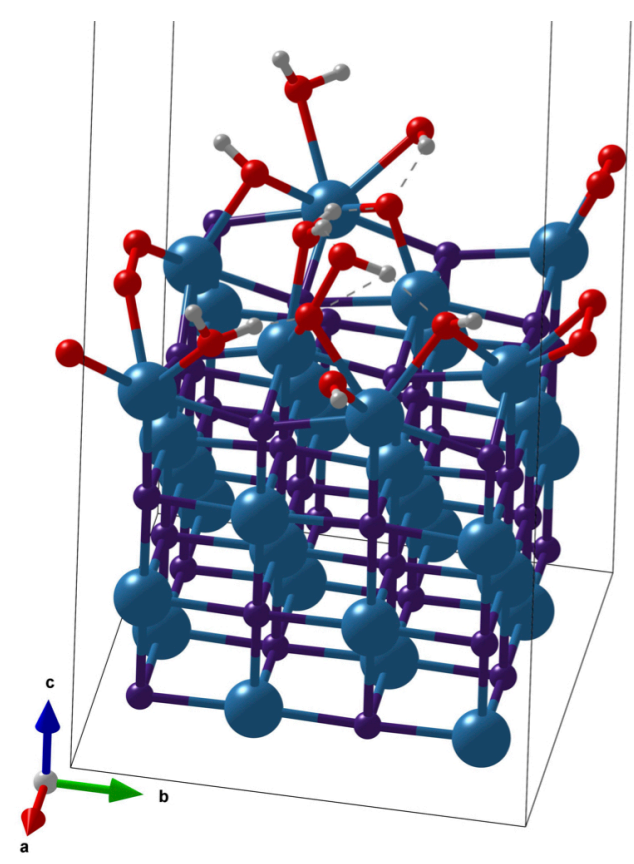

Figure 3.7 UN (100) after $\mathrm{H}_{2} \mathrm{O}$ and $\mathrm{O}_{2}$ adsorption: $\mathrm{U}, \mathrm{N}, \mathrm{O}$, and $\mathrm{H}$ are represented by blue, purple, red, and gray, respectively.

After verifying water dissociation, we added partially dissociative water due to its more favorable adsorption over molecular water as reported by Bo et al [1]. $\mathrm{OH}$ and $\mathrm{O}$ were applied at hollow, N-top, and U-top sites of the UN surfaces: (100) and (110). O adsorbs to $\mathrm{U}$ both in the form of molecular water (w) and hydroxide (h). After the relaxation of UN (100) with 0.5 partially dissociate water coverage, $62.5 \%$ of the 
dissociated $\mathrm{OH}$ and $\mathrm{H}$ revert to $\mathrm{H}_{2} \mathrm{O}$ molecules, preferring to adsorb at hollow sites and coordinate to $U$ atoms with an average bond length $U-O(w)$ of $2.76 \AA$ (Figure 3.5(a) and Figure 3.3(e)). $37.5 \%$ forms $\mathrm{OH}$, where the $\mathrm{O}$ coordinates with the surface due to iondipole interaction. $33 \%$ of $\mathrm{OH}$ bonds at U-top with a U-O (h) bond length of $2.39 \AA$. $66 \%$ of $\mathrm{OH}$ bonds at a hollow site, with $\mathrm{U}-\mathrm{O}(\mathrm{h})$ bond lengths of $2.59,2.42,2.51$, and $2.41 \AA$. $\mathrm{H}_{2} \mathrm{O}$ molecules significantly pull $\mathrm{U}$ away from the surface, with a $3.3 \%$ increase in average distance between terminating $U$ and the second layer compared to the pure UN (100) surface without corrosion.

For the same dissociated water coverage with an added layer of $\mathrm{O}$ at $0.25 \mathrm{ML}$ (Figure 3.5(d) and Figure 3.3(f)), 50\% of $\mathrm{O}$ forms $\mathrm{OH}$ species coordinating with surface U. Of the $\mathrm{OH}$ species, $83 \%$ adsorb at hollow sites and $17 \%$ at $\mathrm{U}$-top sites. The $\mathrm{OH}$ at hollow sites have an average U-O(h) bond length of $2.39 \AA$ and the U-top has a bond length of $2.11 \AA .8 \%$ of the oxygen forms $\mathrm{H}_{2} \mathrm{O}$ and adsorbs to the surface at a U-O(w) distance of $2.57 \AA$. The remaining $\mathrm{O}$ and $\mathrm{H}$ forms water molecules and $\mathrm{H} 2$ that do not adsorb to the surface. With added oxygen, the UN surface is more greatly disrupted with an $11.1 \%$ average increase in the distance between the terminating $\mathrm{U}$ and second layer $\mathrm{U}$.

For the UN (110) surface, the dissociated water gives $1 \mathrm{ML}$ coverage due to the different geometry (Figure 3.5(b) and Figure 3.3(c)). Despite the higher coverage, only $50 \%$ of the $\mathrm{O}$ adsorbs to the surface, both as atomic $\mathrm{O}$ and $\mathrm{H}_{2} \mathrm{O} . \mathrm{O}$ adsorbs at the hollow site with an average U-O distance of $2.14 \AA .75 \%$ of the adsorbed $\mathrm{O}$ attacks the same U atom, pulling it away from the surface. This results in a $3.4 \%$ increase in the average distance between the first and second layer. $6.25 \%$ of $\mathrm{H}$ comes within $2.80 \AA$ of N. 
With added oxygen at $0.5 \mathrm{ML}$ coverage, all terminating $\mathrm{U}$ atoms pull away from the surface, with $25 \%$ of terminating $U$ almost completely removed, attached by only one U-N bond (Figure 3.5(e) and Figure 3.3(h)). This gives an average increase of 15.6\% between the first and second layer. Adsorbates no longer occupy the standard sites due to strong disruption of the terminating layer. $12.5 \% \mathrm{O}$ coordinates with $\mathrm{U}$ at $\mathrm{U}-\mathrm{O}$ distances of 2.15 and $2.20 \AA .12 .5 \% \mathrm{O}$ forms $\mathrm{OH}$ coordinating with $\mathrm{U}$ at $\mathrm{U}-\mathrm{O}(\mathrm{h})$ distances of 2.41 and $2.49 \AA .25 \% \mathrm{OH}$ and $12.5 \%$ water attach to the same $\mathrm{U}$ atom. $37.5 \% \mathrm{OH}$ and $25 \%$ water bonds to the lifted $\mathrm{U}$, with bond lengths ranging from $2.18-2.58 \AA .31 .25 \%$ of $\mathrm{H}$ comes within $2.80 \AA$ of $\mathrm{N}$.

Local density of states (LDOS) in Figure 3.8 shows polar covalent bonds between the dissociated water and the UN surfaces with density overlap (evident of covalent bonding) from approximately -6 to $-2 \mathrm{eV}$ and localization entirely to $\mathrm{UN}$ (evident of ionic bonding) from $-2 \mathrm{eV}$ to the Fermi energy [34]. Figure 3.8(a) is further decomposed into separate $\mathrm{U}$ and $\mathrm{N}$ contributions to illustrate the greater amount of electronic states at $\mathrm{U}$ than N. Examining the PCD resolved from $-5 \mathrm{eV}$ to the Fermi energy on the UN (100) surface (Figure 3.9(a)) shows a smooth distribution of charge at the surface before dissociated water is added. After adding dissociated water in this energy range, the system shows both covalent bond character (Figure 3.9(b-c)) and ionic bond character (Figure 3.9 (e-f)). The presence of charge on both the surface and the adsorbates depicts the covalent bond character, with the contours showing charge density deformation upward towards the adsorbates. Narrowing the partial density of states to $-2 \mathrm{eV}$ to Fermi (Figure 3.9(e-f)) illustrates the polar covalent character with charge localized to the surface and not the adsorbates [35]. As the oxygen concentration increases moving from 
Figure 3.9(d) to (f), the contours show the bonds between the terminating layer and the second layer decreasing, corresponding with the average layer increase discussed above. Similarly, the PCD of the UN (110) surface depicts the periodic surface charge without adsorbates (Figure 3.10(a) and (d)), the covalent bonds formed with the dissociated water seen in the $-5 \mathrm{eV}$ to Fermi range (Figure 3.10(b-c)), and the charge localized to the surface from $-2 \mathrm{eV}$ to the Fermi level (Figure 3.10 (e-f)).

From the ELF, the pure UN (110) surface shows positive character (Figure 3.11(a)), displaying yellow rather than green and blue, characteristic of high electron concentration. This positive character is no longer seen after adsorption (Figure 3.11(b)). The ELF shows large blue regions around $\mathrm{H}_{2}$ indicating an electron sink, with a higher probability of finding electrons there than anywhere else in the system. 

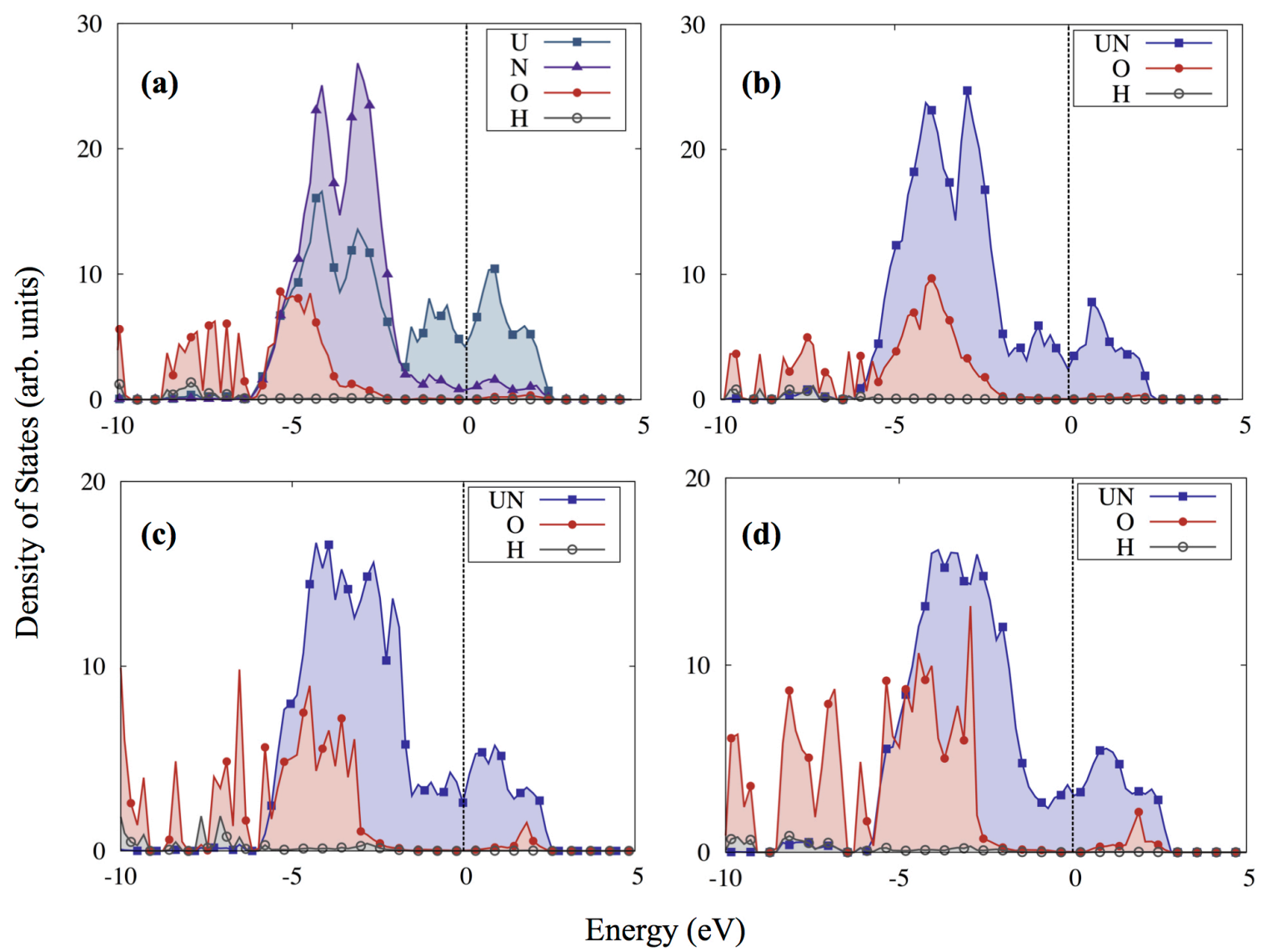

Figure 3.8 Local density of states of (a) UN (100) with $\mathrm{OH}+\mathrm{H}$, (b) UN (100) with $\mathrm{OH}+\mathrm{H}+\mathrm{O}$, (c) $\mathrm{UN}(110) \mathrm{OH}+\mathrm{H}$, and (d) $\mathrm{UN}(110)$ with $\mathrm{OH}+\mathrm{H}+\mathrm{O}$. Individual contributions from $U$ and $N$ are shown in (a), while $U$ and $N$ contributions are combined in (b-d) to indicate bonding between the adsorbates and the surface in general. 


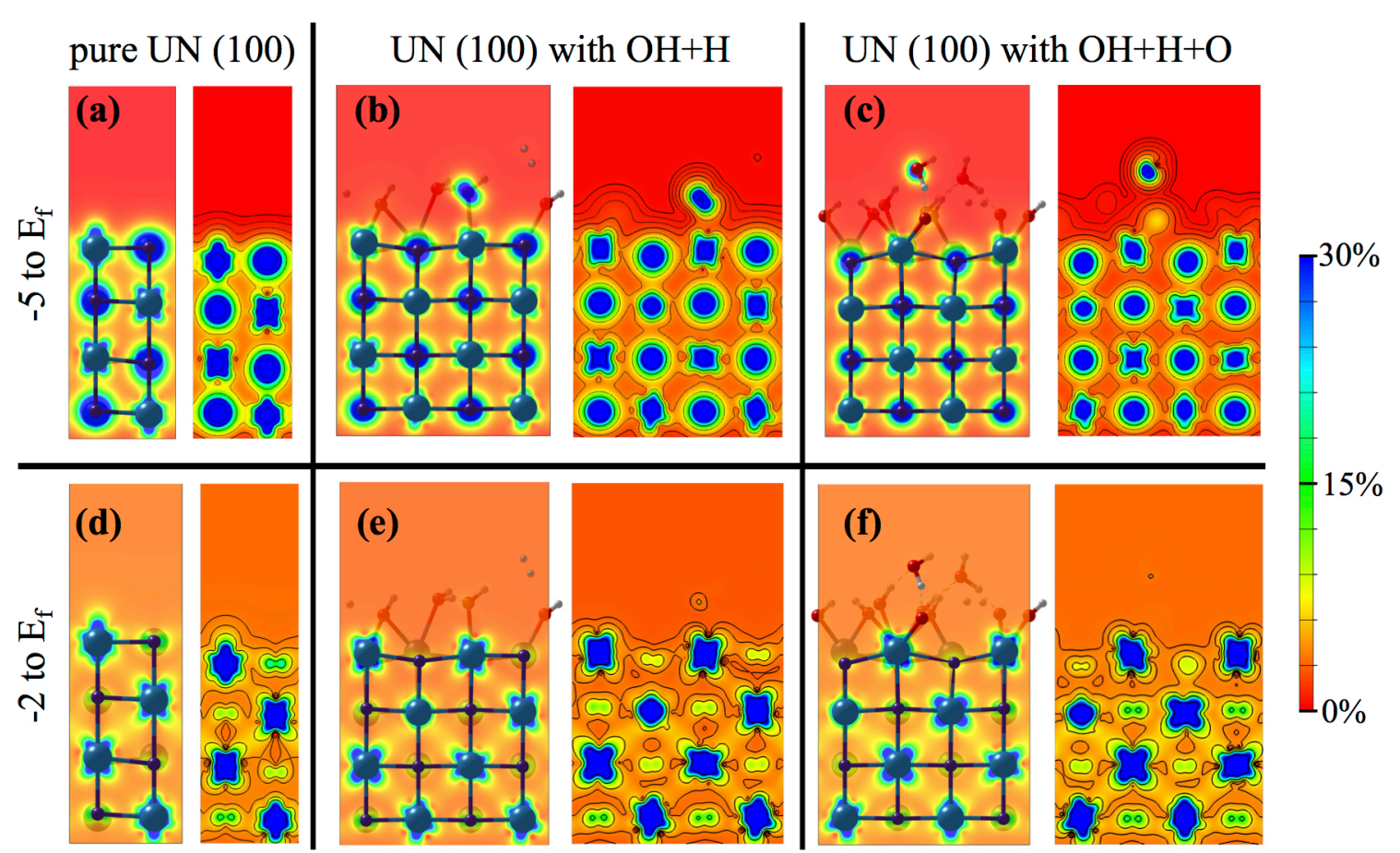

Figure 3.9 Partial charge density of the UN (100) surface without dissociated water, with dissociated water, and with dissociated water and added oxygen. The scale has been narrowed to $30 \%$ of the total electronic states and contour lines added to improve the visibility of bonds. Blue and red indicate many and no electronic states, respectively.

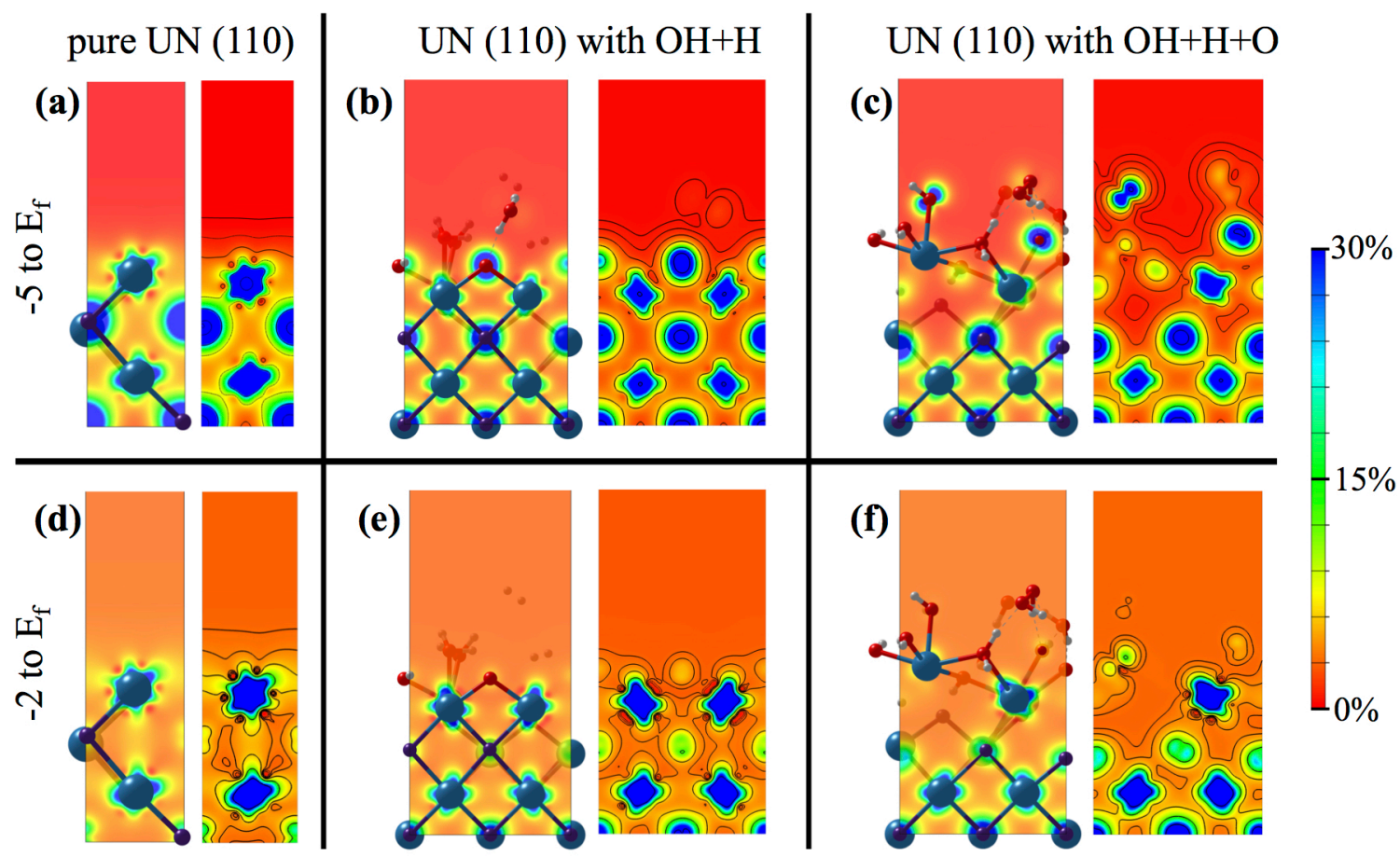

Figure 3.10 Partial charge density of the UN (110) surface without dissociated water, with dissociated water, and with dissociated water and added oxygen. 
(a) pure UN (110)
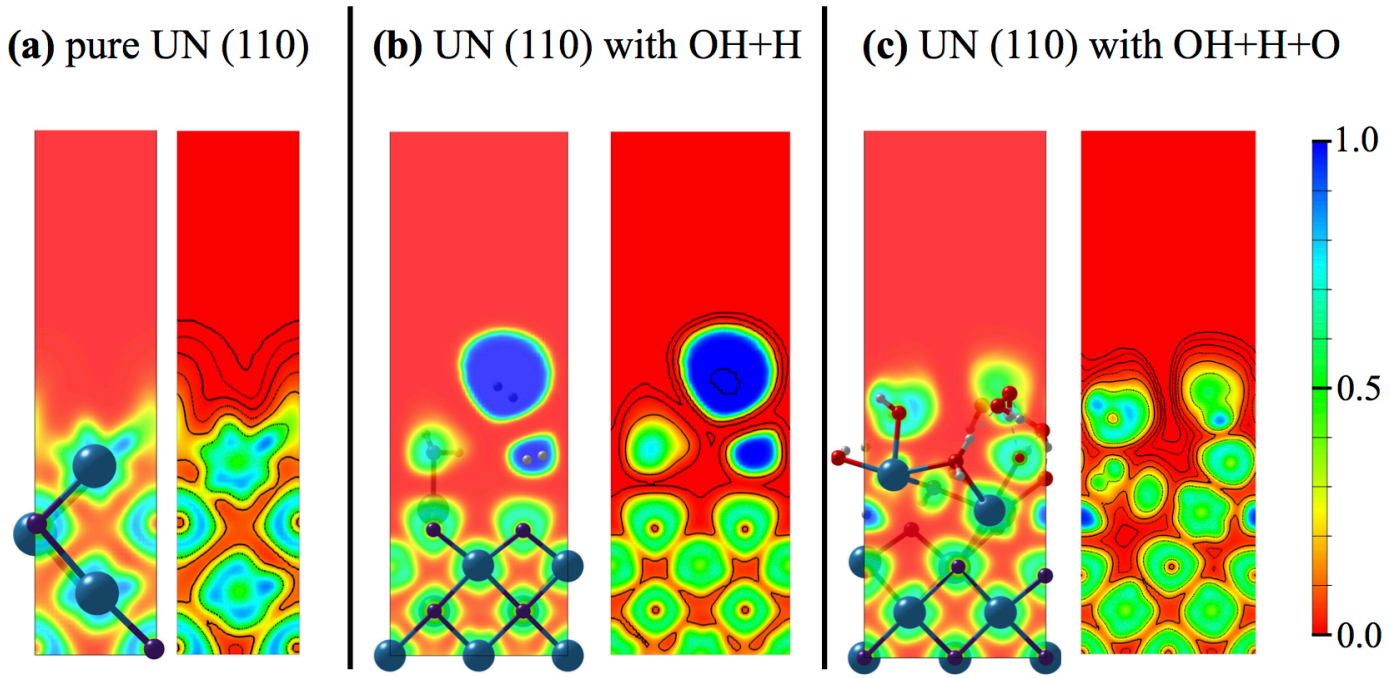

Figure 3.11 Electron localization function of the UN (110) surface without dissociated water, with dissociated water, and with dissociated water and added oxygen.

\subsection{Discussion}

The $\mathrm{Zr} \mathrm{OH}+\mathrm{H}$ system shows at $2 \mathrm{ML}, \mathrm{O}$ adsorbs to the $\mathrm{SFCC}$ site, as previously found in other theoretical studies $[16,17]$. $\mathrm{O}$ also intercalates to the tetrahedral position between the first and second $\mathrm{Zr}$ layers, one of the sites in the lowest energy path of migration in the basal surface determined by Chiang et al [18]. These results are in agreement with the $\mathrm{O}$ positions found from LEED at $2 \mathrm{ML}$ coverage [15].

Consistent with the findings of Bo et al. [1], covalent bonding occurs between $\mathrm{U}$ and $\mathrm{O}$ from approximately $-6 \mathrm{eV}$ to $-2 \mathrm{eV}$ (Figure 3.8). This is similar to the findings of Li et al. [11] depicting overlap between chemisorbed the O2p orbital and $\mathrm{U}$ orbitals $5 f$ and $6 \mathrm{~d}$ from approximately $-5 \mathrm{eV}$ to $-1 \mathrm{eV}$. On the $\mathrm{UN}(100)$ surface, $\mathrm{OH}$ adsorbs either at a hollow site or coordinates with $\mathrm{U}$, as previously reported [1] by Bo et al. Our U-O(h) bonds in the $\mathrm{OH}+\mathrm{H}$ system range from 2.41 to $2.59 \AA$ while the $\mathrm{U}-\mathrm{O}(\mathrm{h})$ bond lengths found by Bo et al. range from 2.15 to $2.72 \AA$. Our $\mathrm{U}-\mathrm{O}(\mathrm{w})$ bond lengths $(2.66,2.69$, and $2.81 \AA)$ are also similar to their 1 ML coverage study $(2.54,2.60$ and $2.71 \AA)$. In contrast 
on the UN (110) surface, Bo et al. [12] found stable partially dissociated water adsorption while our $\mathrm{OH}+\mathrm{H}$ system showed either molecular or completely dissociated water adsorption. The $\mathrm{UN}$ structure in the $\mathrm{OH}+\mathrm{H}+\mathrm{O}$ experienced such distortion that adsorbants could not reasonably be described as adsorbing to a hollow or U-top site. These discrepancies may be due to different degrees of freedom in the surface relaxation, i.e. we allowed the top 2 UN layers to change positions while Bo et al. appeared to have fixed these layers.

Like Zr, UN forms both a positive surface (Zr corrosion mechanism step 3) and relocates charge to $\mathrm{H}$ (step 5) after water adsorption. While $\mathrm{O}$ penetrates the $\mathrm{Zr}$ surface in both the $\mathrm{Zr}-\mathrm{OH}+\mathrm{H}$ and $\mathrm{Zr}-\mathrm{OH}+\mathrm{H}+\mathrm{O}$ systems, $\mathrm{O}$ does not penetrate any of the $\mathrm{UN}$ surfaces. Instead, UOxNy begins to form as U is pulled away from the UN (110) surface. This process appears to stabilize $\mathrm{O}$ as it exhibits charge concentration in the ELF (Figure 3.11(b-c)), but has no active valence electrons (Figure 3.10(e-f)). The effect of dissociated water pulling an atom away from the metal surface is similarly observed in the $\mathrm{Zr}-\mathrm{OH}+\mathrm{H}+\mathrm{O}$ system.

In the dissociated water case, we see stable $\mathrm{H}_{2}$ electron sinks by comparing the ELF (Figure 3.11(b)) and PCD (Figure 3.10(e)). However, in the additional O case, U is pulled further from the UN surface, breaking bonds with all except one of the neighboring $\mathrm{N}$ atoms. $\mathrm{H}$ then travels closer to the surface, coming within $1.75 \AA$ of $\mathrm{N}$ compared to $2.65 \AA$ without partial removal of $U$. While oxide formation and electron relocation to $\mathrm{H}_{2}$ occur sequentially in the $\mathrm{Zr}$ mechanism [14] and form in both $\mathrm{Zr}$ studied systems (Figure 3.5(c and f)), the formation of either $\mathrm{H}_{2}$ sinks (Figure 3.11(b)) or $\mathrm{UO}_{\mathrm{x}} \mathrm{N}_{\mathrm{y}}$ (Figure 3.5(e) and Figure 3.11 (c)) suggests the steps may be competing mechanisms in 
$\mathrm{UN}$ corrosion. Finally, focusing on valence electrons from $-2 \mathrm{eV}$ to the Fermi energy, charge is located almost entirely on the U (Figure 3.8(a) and Figure 3.9 (e-f)). This could explain the results reported by Lu et al. [8] in which N-rich UN films generated thinner oxide layers than $\mathrm{N}$-poor films, as well as the prevalence of residual $\mathrm{N}$ reported across studies. In other words, less electrons are available to react when the majority of the surface is composed of $\mathrm{N}$.

From the above results, we suggest the following UN corrosion mechanisms:

1. Oxynitride formation is initiated with $U$ displacement instead of $O$ intercalation.

2. The UN (110) surface is more active in the corrosion process than the UN (100) surface.

3. Valence electrons from -2 to $0 \mathrm{eV}$ are localized to $\mathrm{U}$.

4. $\mathrm{H}_{2}$ and oxynitride formation are competing reactions.

Mechanism (3) may elucidate why N-rich UN films are less susceptible to corrosion and the prevalence of residual $\mathrm{N}$ across studies. Fewer $\mathrm{N}$ valence electrons are available to react with water and oxygen.

\subsection{Conclusions}

The corrosion mechanism of $\mathrm{Zr}$ is known and relies heavily on differences in electric potential. We used it as a reference to further understand the corrosion mechanism of UN. DFT studies were conducted on UN surfaces (100) and (110) in the presence of dissociated water and additional oxygen and compared to analogous studies on the $\mathrm{Zr}$ (1000) surface. To probe how UN corrosion relies on electric potential, we studied PCD and ELF maps. PCD examined the electrons near the Fermi energy in the 
UN surfaces while ELF took a more cohesive look at the electronic structure through considering all calculated electrons. At the UN surface, as oxygen concentration increased, the distance between the surface layer and bulk increased $11.1 \%$ on the (100) surface and $15.6 \%$ on the (110) surface. PCD maps revealed the bonding between the terminating (100) atomic layer and the bulk weakened when additional oxygen was introduced. Increased oxygen content contributed strongly to $\mathrm{U}$ atom removal. The $\mathrm{Zr}$ structural relaxation and ELF together showed the oxygen adsorption, a positively charged region on the pure zirconium surface, the formation of $\mathrm{ZrO} 2$, and the transfer of electrons to hydrogen, in accordance with the $\mathrm{Zr}$ corrosion mechanism.

Like Zr, the UN corrosion process showed formation of a positively charged surface and charge transfer to $\mathrm{H}$ after dissociated water adsorption. However, while $\mathrm{O}$ spontaneously penetrated the $\mathrm{Zr}$ surface, this process was not seen on the UN surfaces. Instead, UN mimicked Zr corrosion through partial removal of a metal atom when oxygen was present along with dissociated water at the UN (110) surface. Additionally, while $\mathrm{Zr}$ showed both electron transfer to $\mathrm{H}_{2}$ and oxide formation regardless of oxygen concentration, $\mathrm{UN}$ showed either electron transfer to $\mathrm{H}_{2}$ (in the dissociated water case) or oxide formation (in the additional $\mathrm{O}$ case). It implies that electron transfer to $\mathrm{H}_{2}$ and oxide formation might be competing reactions in UN corrosion. The PCD showed charge localized primarily to $\mathrm{U}$ from $-2 \mathrm{eV}$ to the Fermi level. When UN films are N-rich, fewer valence electrons are available to react with water and oxygen. This research suggests that further efforts to increase $\mathrm{N}$ content in $\mathrm{UN}$ may improve corrosion resistance. Our results present the detailed descriptions of $\mathrm{UN}$ and $\mathrm{Zr}$ electronic profiles, towards better 
understanding UN corrosion mechanisms, specifically at the initial stage. This work ultimately contributes to guiding the design of UN for accident tolerant nuclear fuel. Acknowledgements:

This research made use of the resources of the High Performance Computing Center at Idaho National Laboratory, which is supported by the Office of Nuclear Energy of the U.S. Department of Energy and the Nuclear Science User Facilities under Contract No. DE-AC07-05ID14517. Further computational resources were provided by the R2 cluster (DOI: 10.18122/B2S41H) provided by Boise State University’s Research Computing Department. 


\section{References}

[1] T. Bo, J.-H. Lan, Y.-J. Zhang, Y.-L. Zhao, C.-H. He, Z.-F. Chai, W.-Q. Shi, Adsorption and dissociation of $\mathrm{H} 2 \mathrm{O}$ on the (001) surface of uranium mononitride: energetics and mechanism from first-principles investigation, Phys. Chem. Chem. Phys. 18 (2016) 13255-13266, https://doi.org/10.1039/ c6cp01175f.

[2] M. Jolkkonen, P. Malkki, K. Johnson, J. Wallenius, Uranium nitride fuels in superheated steam, J. Nucl. Sci. Technol. J. J. Nucl. Sci. Technol. 54 (2017) 5131519, https://doi.org/10.1080/00223131.2017.1291372.

[3] D. Bocharov, D. Gryaznov, Y.F. Zhukovskii, E.A. Kotomin, Ab initio simulations of oxygen interaction with surfaces and interfaces in uranium mononitride, J. Nucl. Mater. 435 (2013) 102-106, https://doi.org/10.1016/j.jnucmat.2012.12.031.

[4] D.A. Lopes, A. Claisse, P. Olsson, Ab-initio study of C and $\mathrm{O}$ impurities in uranium nitride, J. Nucl. Mater. 478 (2016) 112-118, https://doi.org/10.1016/ J.JNUCMAT.2016.06.008.

[5] R.M. Dell, V.J. Wheeler, N.J. Bridger, Hydrolysis of uranium mononitride, Trans. Faraday Soc. 63 (1967) 1286, https://doi.org/10.1039/tf9676301286.

[6] S. Sugihara, S. Imoto, Hydrolysis of uranium nitrides, J. Nucl. Sci. Technol. 6 (1969) 237-242, https://doi.org/10.1080/18811248.1969.9732878.

[7] K. Liu, L. Luo, L. Luo, Z. Long, Z. Hong, H. Yang, S. Wu, Initial oxidation behaviors of nitride surfaces of uranium by XPS analysis, Appl. Surf. Sci. 280 (2013) 268-272, https://doi.org/10.1016/j.apsusc.2013.04.147.

[8] L. Lu, F. Li, Y. Hu, H. Xiao, B. Bai, Y. Zhang, L. Luo, J. Liu, K. Liu, The initial oxidation behaviors of uranium nitride $\mathrm{UNx}(\mathrm{x}=0,0.23,0.68,1.66)$ films, $\mathrm{J}$. Nucl. Mater. 480 (2016) 189-194, https://doi.org/10.1016/J.JNUCMAT.2016.08.025. 
[9] Z. Long, R. Zeng, Y. Hu, J. Liu, W. Wang, Y. Zhao, Z. Luo, B. Bai, X. Wang, K. Liu, Electronic structure and fine structural features of the air-grown UNxOy on nitrogen-rich uranium nitride, Appl. Surf. Sci. 443 (2018) 407-411, https:// doi.org/10.1016/J.APSUSC.2018.02.271.

[10] D. Bocharov, D. Gryaznov, Y.F. Zhukovskii, E.A. Kotomin, Ab initio modeling of oxygen impurity atom incorporation into uranium mononitride surface and subsurface vacancies, J. Nucl. Mater. 416 (2011) 200-204, https://doi.org/ 10.1016/j.jnucmat.2010.11.090.

[11] R.-S. Li, X. Peng, W. Fei, M. Wen-Yan, Density functional theory study of H, C and O chemisorption on UN(001) and (111) surfaces, Nucl. Sci. Tech. 25 (2014), https://doi.org/10.13538/j.1001-8042/nst.25.050502, 050502-10.

[12] T. Bo, J.-H. Lan, Y.-L. Zhao, C.-H. He, Z.-F. Chai, W.-Q. Shi, First-principles study of water reacting with the (110) surface of uranium mononitride, J. Nucl. Mater. 492 (2017) 244-252, https://doi.org/10.1016/J.JNUCMAT.2017.05.026.

[13] B. Cox, Some thoughts on the mechanisms of in-reactor corrosion of zirconium alloys, J. Nucl. Mater. (2005) 331-368, https://doi.org/10.1016/ j.jnucmat.2004.09.029.

[14] A.T. Motta, A. Couet, R.J. Comstock, Corrosion of zirconium alloys used for nuclear fuel cladding, Annu. Rev. Mater. Res. 45 (2015) 311-343, https:// doi.org/10.1146/annurev-matsci-070214-020951.

[15] Y.M. Wang, Y.S. Li, K.A.R. Mitchell, LEED crystallographic analysis for the structure formed by 2 ML of O at the $\operatorname{Zr}(0001)$ surface, Surf. Sci. 380 (1997) 540547, https://doi.org/10.1016/S0039-6028(97)00039-3.

[16] F.-H. Wang, S.-Y. Liu, J.-X. Shang, Y.-S. Zhou, Z. Li, J. Yang, Oxygen adsorption on $\operatorname{Zr}\left(\begin{array}{llll}0 & 0 & 0 & 1\end{array}\right)$ surfaces: density functional calculations and a multiple-layer adsorption model, Surf. Sci. 602 (2008) 2212-2216, https://doi.org/10.1016/ J.SUSC.2008.04.033. 
[17] G. Jomard, A. Pasturel, On the effect of oxygen adsorption on the interlayer relaxation of the $\operatorname{Zr}\left(\begin{array}{llll}0 & 0 & 0 & 1\end{array}\right)$ surface, Appl. Surf. Sci. 177 (2001) 230-237, https://doi.org/10.1016/S0169-4332(01)00217-3.

[18] T.-W. Chiang, A. Chernatynskiy, M.J. Noordhoek, S.B. Sinnott, S.R. Phillpot, Anisotropy in oxidation of zirconium surfaces from density functional theory calculations, Comput. Mater. Sci. 98 (2015) 112-116, https://doi.org/10.1016/ j.commatsci.2014.10.052.

[19] G. Kresse, J. Furthmüller, Efficient iterative schemes for ab initio total-energy calculations using a plane-wave basis set, Phys. Rev. B 54 (1996) 11169-11186, https://doi.org/10.1103/PhysRevB.54.11169.

[20] J.P. Perdew, K. Burke, M. Ernzerhof, Gen. Gradient Aprox. Made Simple 77 (1996) 3865-3868, https://doi.org/10.1103/PhysRevLett.77.3865.

[21] S.L. Dudarev, G.A. Botton, S.Y. Savrasov, Z. Szotek, W.M. Temmerman, A.P. Sutton, Electronic structure and elastic properties of strongly correlated metal oxides from first principles: LSDA p U, SIC-lsda and EELS study of UO2 and NiO, Phys. Status Solidi 166 (1998) 429-443, https://doi.org/10.1002/ (SICI)1521-396X(199803)166:1<429. AID-PSSA429>3.0.CO;2-F.

[22] Y. Lu, B.-T. Wang, R.-W. Li, H.-L. Shi, P. Zhang, Structural, electronic, mechanical, and thermodynamic properties of UN2: systematic density func- tional calculations, J. Nucl. Mater. 410 (2011) 46-51, https://doi.org/10.1016/ J.JNUCMAT.2010.12.308.

[23] B. Silvi, A. Savin, Classification of chemical bonds based on topological analysis of electron localization functions, Lett. to Nat. 371 (1994) 683-686, https:// doi.org/10.1038/371683a0.

[24] T.H. da Silva, E.B. Nelson, I. Williamson, C.M. Efaw, E. Sapper, M.F. Hurley, L. Li, First-principles surface interaction studies of aluminum-copper and aluminumcopper-magnesium secondary phases in aluminum alloys, Appl. Surf. Sci. 439 (2018) 910-918, https://doi.org/10.1016/J.APSUSC.2017.12.256. 
[25] P. Fuentealba, E. Chamorro, J.C. Santos, Chapter 5: understanding and using the electron localization function, in: Theor. Asp. Chem. React, Elsevier, 2007, pp. 57-85, https://doi.org/10.1016/S1380-7323(07)80006-9.

[26] H.W. Knott, G.H. Lander, M.H. Mueller, O. Vogt, Search for lattice distortions in UN, UAs, and USb at low temperatures, Phys. Rev. B 21 (1980) 4159-4165, https://doi.org/10.1103/PhysRevB.21.4159.

[27] A. Claisse, M. Klipfel, N. Lindbom, M. Freyss, P. Olsson, GGApU study of uranium mononitride: a comparison of the U-ramping and occupation matrix schemes and incorporation energies of fission products, J. Nucl. Mater. 478 (2016) 119-124, https://doi.org/10.1016/J.JNUCMAT.2016.06.007.

[28] D. Rafaja, L. Havela, R. Ku zel, F. Wastin, E. Colineau, T. Gouder, Real structure and magnetic properties of UN thin films, J. Alloy. Comp. 386 (2005) 87-95, https://doi.org/10.1016/J.JALLCOM.2004.06.020.

[29] D. Bocharov, D. Gryaznov, Y.F. Zhukovskii, E.A. Kotomin, DFT calculations of point defects on UN(001) surface, Surf. Sci. 605 (2011) 396-400, https:// doi.org/10.1016/J.SUSC.2010.11.007.

[30] Y. Lu, B.-T. Wang, R.-W. Li, H. Shi, P. Zhang, Structural, Electronic, and Thermodynamic Properties of UN: Systematic Density Functional Calculations, 2010, pp. 218-222, https://doi.org/10.1016/j.jnucmat.2010.08.026.

[31] R.A. Evarestov, A.V. Bandura, M.V. Losev, E.A. Kotomin, Y.F. Zhukovskii, D. Bocharov, A first-principles DFT study of UN bulk and (001) surface: comparative LCAO and PW calculations, J. Comput. Chem. 29 (2008) 20792087, https://doi.org/10.1002/jcc.21023.

[32] P.W. Tasker, The stability of ionic crystal surfaces, J. Phys. C Solid State Phys. 12 (1979) 4977-4984, https://doi.org/10.1088/0022-3719/12/22/036.

[33] W. Sun, G. Ceder, Efficient creation and convergence of surface slabs, Surf. Sci. 617 (2013) 53-59, https://doi.org/10.1016/J.SUSC.2013.05.016. 
[34] L. Li, Y.-N. Wu, H.-P. Cheng, First-principles calculations of Fe-doped mono- layer C60 on h-BN/Ni(111) surface, J. Appl. Phys. 132 (2010) 1-11, https:// doi.org/10.1063/1.3291080.

[35] L.M. Liu, S.Q. Wang, H.Q. Ye, First-principles study of polar A1/TiN(1 11 1) interfaces, Acta Mater. 52 (2004) 3681-3688, https://doi.org/10.1016/ j.actamat.2004.04.022. 


\section{CHAPTER FOUR: MAGNETIC PROPERTIES OF THE URANIUM NITRIDE SURFACE}

This chapter has been accepted for publication by AIP Publishing in Journal of Applied Physics. 
Magnetic Properties of the Uranium Nitride Surface

\author{
Ember L. Sikorski $i^{1,2}$ \\ Brian J. Jaques ${ }^{1,2}$ \\ Lan $\mathrm{Li}^{1,2}$ \\ ${ }^{1}$ Micron School of Materials Science and Engineering, \\ Boise State University, Boise, ID 83625, USA \\ ${ }^{2}$ Center for Advanced Energy Studies, Idaho Falls, ID 83401, USA
}




\begin{abstract}
The magnetic properties of UN surfaces are not well understood experimentally or computationally, but they have a significant effect on UN performance as a nuclear fuel. We investigated ferromagnetic (FM), antiferromagnetic (AFM), nonmagnetic (NM), and three hybrid magnetic structures of the most stable UN surface (100). To account for electron correlation and metastability, a U-ramp was performed to an effective Hubbard U-term of $2.0 \mathrm{eV}$. FM was found to be the most energetically favorable magnetic structure. Type 1 AFM slab was optimized to a new magnetic structure consisting of (100) planes with either all spin-up electrons, all spin-down electrons, or half spin-up and half spin-down electrons on uranium atoms. After $\mathrm{OH}$ adsorption to simulate corrosion initiation, the AFM, FM, and NM structures yield relatively similar bond lengths but varying bond angles, adsorption energies, and electronic profiles. Partial Charge Density maps show varying degradation mechanisms across magnetic structures. Electron Localization Function reveals more charge localized to AFM uranium atoms with spindown electrons than uranium atoms with spin-up electrons. This leads to different properties depending on if an adsorbate interacts with a spin-up or spin-down terminated AFM surface. This work supports the physical accuracy of future computational studies towards corroborating with experiment and addressing UN fuel corrosion.
\end{abstract}

\title{
4.1 Introduction
}

Uranium Nitride (UN) is proving to be a promising candidate nuclear fuel due to its high actinide density, thermal conductivity, and melting point [1]. One barrier for implementation is its instability in the presence of water. Several experimental [2-6] studies have sought to determine the mechanism of UN corrosion, though there is not yet 
a consensus on the chemical reactions that occur. Dell et al. [2] first proposed the UN corrosion mechanism as $\mathrm{UN}+2 \mathrm{H}_{2} \mathrm{O} \rightarrow \mathrm{UO}_{2}+\mathrm{NH}_{3}+\frac{1}{2} \mathrm{H}_{2}$. However, Dell et al. noted that there was dissolved $\mathrm{N}$ as well as a $\mathrm{U}_{2} \mathrm{~N}_{3+\mathrm{x}}$ phase in the corrosion product. Subsequent experimental studies have continued to report the presence of $\mathrm{UN}_{2}, \mathrm{U}_{2} \mathrm{~N}_{3}$, and/or residual $\mathrm{N}$ in the product [3-6]. This suggests that the initially proposed corrosion mechanism is incomplete in the hypothesis that all $\mathrm{N}$ is converted into $\mathrm{NH}_{3}$. To complement experimental studies, Density Functional Theory (DFT) has been a common method used to study $\mathrm{UN}$ surfaces exposed to $\mathrm{H}_{2} \mathrm{O}$ or $\mathrm{O}_{2}$ at the atomic scale [7-12]. While experimental studies might utilize UN reactants of varying density, purity, and geometries, DFT allows for precise selection of the reactant. DFT can also be used to further investigate atomic corrosion initiation mechanisms.

However, these DFT studies have applied different magnetic structures to the UN surface. This variation in the magnetic treatment of the surface might significantly affect the simulations, highlighting the uncertainty of prediction of the chemical reactions occurring during the corrosion. As we develop our understanding of UN corrosion, the effect of magnetic structure on such properties should be further investigated. Additionally, without agreement upon the magnetic structure, DFT data could be translated to inaccurate descriptions of larger length scale phenomena.

The variations in magnetic treatments to UN surfaces in DFT studies stem from disagreement over whether a UN surface has the same magnetic structure as UN bulk. In bulk, UN is type 1 antiferromagnetic (AFM), such that (100) planes alternate between positive and negative spin [13]. However, it has been observed in DFT studies that for UN slabs, typically ranging from three to eleven monolayers (approximately 7 to $27 \AA$ ), 
ferromagnetic (FM) structure is more stable than AFM structure [7,14,15]. Likewise, FM treatment has yielded more energetically favorable adsorption energies [16]. However, these studies have not included the Hubbard U-term in their magnetic consideration. In traditional DFT, electrons are systematically delocalized, which can lead to inaccurate descriptions for strongly correlated materials including actinides [17]. These descriptions can be improved with the incorporation of a Coulomb repulsion U-term for f electrons following the ideas of Hubbard $[18,19]$. For UN, it has been found necessary to include the U-term in DFT calculations to reproduce lattice parameter, bulk modulus, phonon properties, magnetic structure, and magnetic moment in good agreement with experiments [20,21]. Gryaznov et al. showed that as the U-term was increased, the most stable UN bulk magnetic structure transitioned from FM to AFM above an effective Uterm, $U_{\text {eff, }}$ of $1.65 \mathrm{eV}[21]$.

Three different strategies have been reported for treating the UN surface magnetic structure in the DFT calculations. Bo et al. used AFM treatment in order to replicate the most stable magnetic structure of the bulk [11]. Li et al. suggested nonmagnetic treatment was appropriate for only total energy calculations [9]. Most prevalently, a few studies used FM treatment $[8,12,16,22]$ citing FM as the most energetically favorable structure. In experiments, Rafaja et al. studied the magnetic susceptibility of reactive sputtered UN thin films, suggesting FM for UN crystallites (averaging $17 \mathrm{~nm}$ )[23]. Bright et al. studied an epitaxial UN film (70 nm) using x-ray synchrotron techniques and reported an AFM structure [24]. However, both experimental studies treated thicker UN systems (over 100 Å thicker) than the DFT studies did. 
In our previous work, we studied UN (100) and (110) surfaces using DFT.[12] In agreement with Tasker's analysis [25] and other DFT studies $[9,10]$, we found the $(100)$ surface to be the most energetically favorable. In order to comprehensively understand the magnetic structure of UN and its surface effect, this work investigates three key aspects. First is to identify the most stable magnetic structure of UN (100) in consideration with the Hubbard U-term. Second is to evaluate if a transition between FM and AFM structure is favorable. Final is to determine the effects of different magnetic structures on bond lengths, bond angles, adsorption energies, and electronic mapping.

\subsection{Methods}

DFT calculations were performed using the Vienna Ab initio Simulation Package (VASP) [26]. Spin-polarized generalized gradient (GGA) exchange-correlation functional was used with the Perdew Burke Ernzerhoff (PBE) formulation [27]. Plane-wave basis sets were implemented utilizing projector-augmented wave (PAW) pseudopotentials. Simulations were performed with a cutoff energy of $550 \mathrm{eV}$ and $4 \times 4 \times 1$ gamma-centered k-points.

UN slabs were constructed with 8 monolayers and a surface area of 16 atoms, i.e. the UN unit cell was extended by factors of $2 \times 2 \times 4$ to form supercells. In our previous work, we found trends in electronic mapping to be converged for 4 monolayers, but binding energies varied between 4 and 8 monolayer systems by up to approximately 0.5 eV [12]. Similarly, Bocharov et al. found defect energies to be converged for slabs with 7 or more monolayers [22]. Bo et al. compared defect energies, surface energies, and bond lengths between supercells with surface areas corresponding to $2 \times 2$ and $3 \times 3$ unit cells and found the $2 \times 2$ surface area to be sufficient for corrosion studies [11]. 
The U-term was applied using the Dudarev implementation [17]. In this version, only the effective U-term, $U_{\text {eff }}=U-J$, between the Hubbard $\mathrm{U}$ and exchange parameter $\mathrm{J}$ is considered. The $\mathrm{J}$ parameter was fixed at $0.5 \mathrm{eV}$ and the $\mathrm{U}$ parameter was varied to reach the desired $U_{\text {eff. }}$. While the U-term improves the DFT description of actinides, it introduces an additional concern of converging to metastable states [28]. As such, calculations may not always reach the global energetic minima, which can lead to errors in the calculations. The so-called "U-ramping" method is one technique that has been developed to better find the ground state [29]. In this method, $U_{\text {eff }}$ is gradually increased from zero to the desired value in increments of $0.1 \mathrm{eV}$. For each step, the previous atomic coordinates and wave functions are used as the initial guess for a new calculation.

Gryaznov et al. showed that the stable magnetic structure of the UN bulk, either FM or AFM, depended on the $U_{\text {eff }}$ value. Therefore, we performed a U-ramp on the UN (100) slabs to not only treat the metastability but also survey the most favorable magnetic structure over a range of $U_{\text {eff }}$ values. Previous UN corrosion studies used a $U_{\text {eff }}$ value of $1.9 \mathrm{eV}$ [10-12]. This value could balance the $1.85 \mathrm{eV}$ recommendation from Gryaznov et al. [21] for magnetic properties and the recommendation of $2.0 \mathrm{eV}$ from $\mathrm{Lu}$ et al. [20] for lattice parameter, bulk modulus, and phonon properties. To encompass all these recommended $U_{\text {eff }}$ values, the U-ramp was performed up to $2.0 \mathrm{eV}$ in our studies. During the U-ramp, slabs were relaxed symmetrically, i.e. the center 2 monolayers were fixed, to prevent any unphysical polarity from affecting the favorability of each magnetic structure. An optimization of a single $\mathrm{OH}$ adsorbate in the $2 \times 2 \times 4 \mathrm{UN}$ (100) supercell, i.e. $1 / 8$ surface coverage, was performed. The bottom 2 layers of the UN slab were fixed to simulate the bulk region. Since our previous work demonstrated the sensitivity of 
adsorption energy to slab thickness [12], asymmetric surface relaxation was utilized to ensure adsorption energy accuracy.

\subsection{Results}

\subsubsection{Magnetic Structure}

With bulk UN exhibiting AFM structure and surfaces exhibiting FM structure, it would be crucial to determine a transition mechanism from AFM to FM. We tested AFM, FM, and another three possible hybrid magnetic structures for comparison, as seen in Figure 4.1. Using symmetrical slabs, the central layers represent the bulk and as such we applied AFM structure of varying thicknesses for the hybrid configurations. Hybrid Configuration (HC) 1 has the greatest AFM character with 6 central AFM monolayers, leaving 2 FM monolayers at each terminating surface. HC2 has only 2 central AFM monolayers with 4 FM monolayers on either side. HC3 has 4 central AFM layers and 3 FM monolayers on either side.

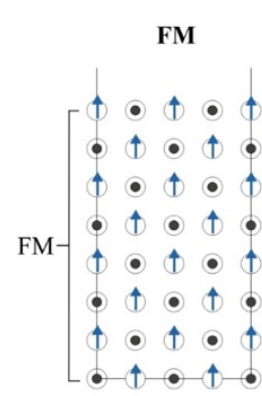

Figure 4.1
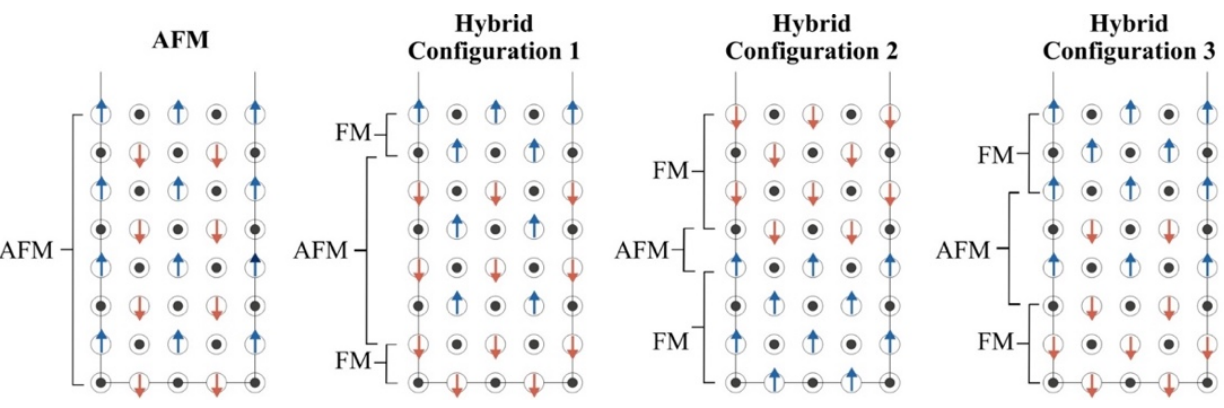

Initial magnetic structures applied during $U$-ramp to $U_{\text {eff }}=2.0 \mathrm{eV}$.

For each magnetic structure, we performed a U-ramp to $2.0 \mathrm{eV}$, which included the recommended values to accurately reproduce UN bulk magnetism, lattice parameter, bulk modulus, and phonon properties [20,21]. Figure 4.2 shows the relative energies for each magnetic structure with reference to the most energetically favorable magnetic structure (FM) during the U-ramp. Without the U-term (i.e. $U_{\text {eff }}=0 \mathrm{eV}$ ), FM is the most 
stable while AFM is the least stable, in agreement with Zhukovskii et al. $[14,15]$ and Evarestov et al.[30] Before the U-ramp, FM is more stable than AFM by $2.66 \mathrm{eV}$. For the duration of the U-ramp, FM remains the most stable magnetic structure, unlike bulk UN that would transition from FM to AFM at $U_{\text {eff }}=1.65 \mathrm{eV}$ [21]. There are a couple of transitions in the order of the magnetic configuration stability of the HC structures. At $U_{\text {eff }}=1.6 \mathrm{eV}, \mathrm{HC} 1$ becomes more stable than $\mathrm{HC} 3$. At $U_{\text {eff }}=2.0 \mathrm{eV}, \mathrm{HC} 1$ becomes more stable than $\mathrm{HC} 2$. At $U_{\text {eff }}=2.0 \mathrm{eV}$, the magnetic structures from the most to the least stable are FM, HC1, HC2, HC3, and AFM.

Except for AFM, the initial magnetic structures (Figure 4.1) are maintained for the duration of the U-ramp, as shown in Table 4.1. FM, HC1, HC2, and HC3 exhibit that the magnitudes of the magnetic moments in layers 1 through 4 mirror those in layers 5 through 8 . The higher magnitudes of the magnetic moments occur in the outer layers 1 and 8 and gradually decrease moving toward the central layers 4 and 5 . Conversely, for AFM some of the electrons of the $U$ atoms flip their spins at $U_{\text {eff }}=1.6 \mathrm{eV}$ in layers 3,5 , 7, and 8. Layers 3, 5, and 7 started as spin-down and the electrons of half of the $U$ atoms flipped to spin-up. Layer 8 started as spin-up and half of the electrons of the $U$ atoms flipped to spin-down. This results in layers 3, 5, 7, and 8 exhibiting internal AFM structures with half the $U$ atoms with spin-up electrons and half with spin-down electrons. At the end of the U-ramp $\left(U_{\text {eff }}=2.0 \mathrm{eV}\right)$ this new AFM structure is less stable than the FM structure by $0.91 \mathrm{eV}$. 


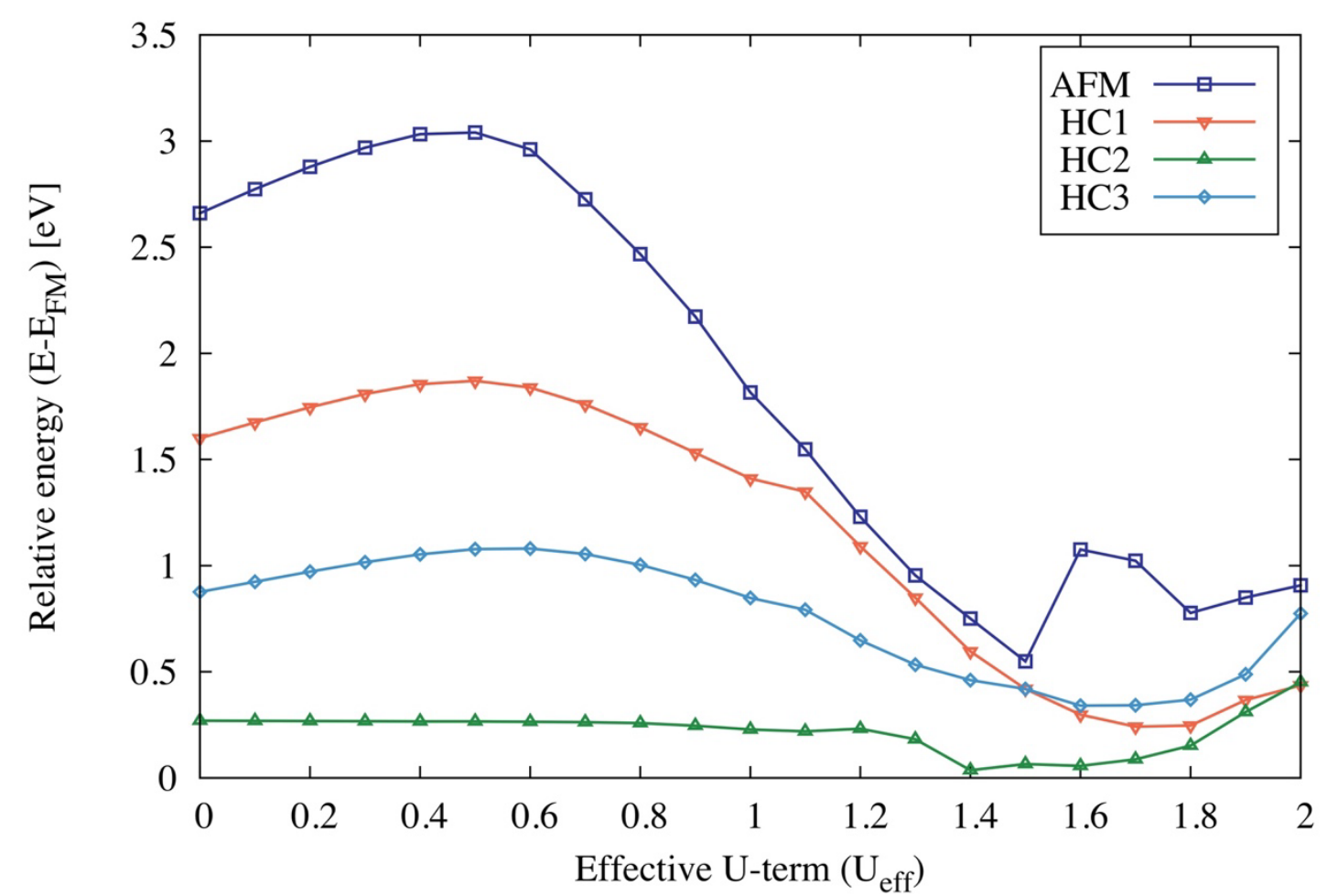

Figure 4.2 Relative energy (eV) of the magnetic structures with respect to $U_{\text {eff }}$ during the U-ramp to $2.0 \mathrm{eV}$. Energies are given with respect to the FM total energy at each respective $U_{\text {eff }}$ value. Note that the AFM values correspond to the structure that was initialized as type 1 AFM, corresponding to bulk $U N$, before the U-ramp but transitioned to a new magnetic structure as shown in Table 4.1. 
Table 4.1 Magnetic moments $\left(\mu_{B}\right)$ of $U$ atoms averaged over each (100) layer after U-ramping to $\mathrm{U}_{\text {eff }}=\mathbf{2 . 0} \mathrm{eV}$.

\begin{tabular}{lccccc}
\hline & FM & AFM & HC1 & HC2 & HC3 \\
\hline Layer 1 & 2.10 & -2.07 & -2.11 & 2.09 & 2.07 \\
Layer 2 & 1.93 & 1.46 & -1.79 & 1.89 & 1.85 \\
Layer 3 & 1.79 & 0.01 & 1.89 & 1.75 & 1.47 \\
Layer 4 & 1.72 & 1.69 & -1.72 & 1.57 & -1.80 \\
Layer 5 & 1.74 & 0.42 & 1.84 & -1.52 & 1.80 \\
Layer 6 & 1.75 & -1.26 & -1.68 & -1.76 & -1.47 \\
Layer 7 & 1.89 & -0.07 & 1.77 & -1.89 & -1.85 \\
Layer 8 & 2.10 & -0.01 & 2.09 & -2.09 & -2.07 \\
\hline
\end{tabular}




\section{$\underline{4.3 .2 \text { Electronic Structure }}$}

To understand the effect of magnetic structures on corrosion results, we relaxed $\mathrm{OH}$ at the U-top site of the three magnetic structures used in the literature: FM, AFM, and $\mathrm{NM}$, as seen in Figure 4.3. OH serves as a critical step in corrosion at UN surfaces in the first-principles UN studies.[10-12,16] OH adsorption is most favorable at the U-top site [10]. In order to determine the effect of electron-spin polarization on adsorption properties, $\mathrm{OH}$ was studied at both AFM spin-up and spin-down terminated surfaces. The most readily apparent difference in $\mathrm{OH}$ adsorption across structures is the variation in $\mathrm{O}$ U-N bond angles. Additional bond angles, as well as bond lengths, vertical displacement of the $\mathrm{U}$ atoms bonded to $\mathrm{O}$, and adsorption energies are given in Table 4.2.

All the magnetic treatments yield identical O-H and $\mathrm{O}-\mathrm{U}$ bond lengths, except for NM, which yields a $0.05 \AA$ shorter O-U bond length. The H-O-U bond angles are obtuse for the spin-polarized structures and vary at most by $1.67^{\circ}$, while NM yields a $180^{\circ}$ bond angle. The O-U-N bond angles vary significantly more across spin-polarized structures with differences up to $10.39^{\circ}$. FM predicts a U displacement of $0.39 \AA$, while NM underpredicts the displacement by $0.19 \AA$ and both AFM structures overpredict the displacement by at least $0.20 \AA$. NM predicts the greatest magnitude of adsorption energy, FM the smallest, and both AFM structures lie in the middle. 
a) FM

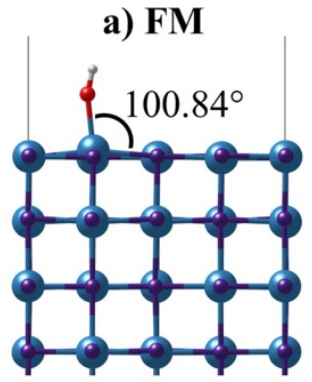

b) AFM-up

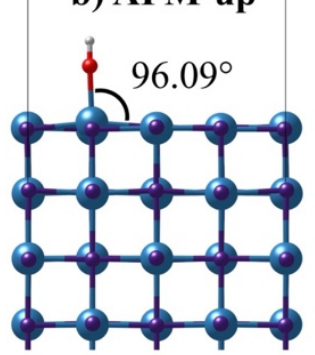

c) AFM-down

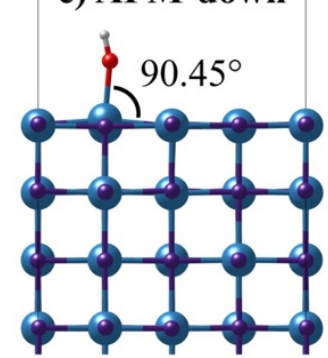

d) $\mathbf{N M}$

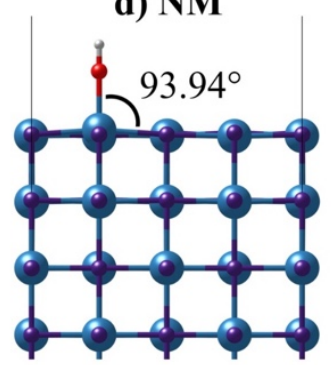

Figure 4.3 Atomic structures after $\mathrm{OH}$ adsorption for FM, AFM spin-up terminated, AFM spin-down terminated, and NM structures. Blue, purple, red, and white atoms represent $U, N, O$, and $H$, respectively.

Table 4.2 Bond lengths, bond angles, vertical displacement of the $U$ atom bonded to $\mathrm{OH}$ after geometry optimization, and adsorption energy for FM, AFM spin-up terminated, AFM spin-down terminated, and NM magnetic structures. Two $\mathrm{O}-\mathrm{U}-\mathrm{N}$ bond angles are reported for each structure: one considering the neighboring $\mathbf{N}$ atom pictured to the right and one considering the $\mathbf{N}$ atom into the page in Figure 4.3.

\begin{tabular}{|c|c|c|c|c|c|c|c|}
\hline & $\begin{array}{l}\text { O-U bond } \\
\text { length }(\AA)\end{array}$ & $\begin{array}{l}\text { O-H bond } \\
\text { length }(\AA)\end{array}$ & $\begin{array}{l}\mathrm{H}-\mathrm{O}-\mathrm{U} \\
\text { bond angle } \\
\left({ }^{\circ}\right)\end{array}$ & $\begin{array}{l}\text { O-U-N } \\
\text { right angle } \\
\left({ }^{\circ}\right)\end{array}$ & $\begin{array}{l}\text { O-U-N into } \\
\text { page angle } \\
\left({ }^{\circ}\right)\end{array}$ & $\begin{array}{l}\mathrm{U} \\
\text { displacement } \\
(\AA)\end{array}$ & $\begin{array}{l}\text { Adsorption } \\
\text { Energy }(\mathrm{eV})\end{array}$ \\
\hline FM & 2.17 & 0.97 & 162.05 & 100.84 & 95.65 & 0.39 & -4.41 \\
\hline $\begin{array}{l}\text { AFM- } \\
\text { up }\end{array}$ & 2.17 & 0.97 & 163.72 & 96.09 & 100.64 & 0.62 & -4.56 \\
\hline $\begin{array}{l}\text { AFM- } \\
\text { down }\end{array}$ & 2.17 & 0.97 & 162.82 & 90.45 & 96.17 & 0.60 & -4.83 \\
\hline NM & 2.12 & 0.97 & 180.00 & 93.94 & 93.94 & 0.20 & -5.23 \\
\hline
\end{tabular}

The Local Densities of States (LDOS) for OH adsorbed on the FM, AFM, and NM slabs, shown in Figure 4.4, depict relatively similar trends. Valence and conduction electrons are localized primarily on $\mathrm{U}$ and $\mathrm{N}$. More electrons are localized on $\mathrm{N}$ than $\mathrm{U}$ from approximately -6 to $-2 \mathrm{eV}$, while more electrons are on $\mathrm{U}$ than $\mathrm{N}$ from approximately -2 to $2 \mathrm{eV}$. Hybridization of $\mathrm{O}$ with $\mathrm{U}$ and $\mathrm{N}$ occurs around $-4 \mathrm{eV}$. 

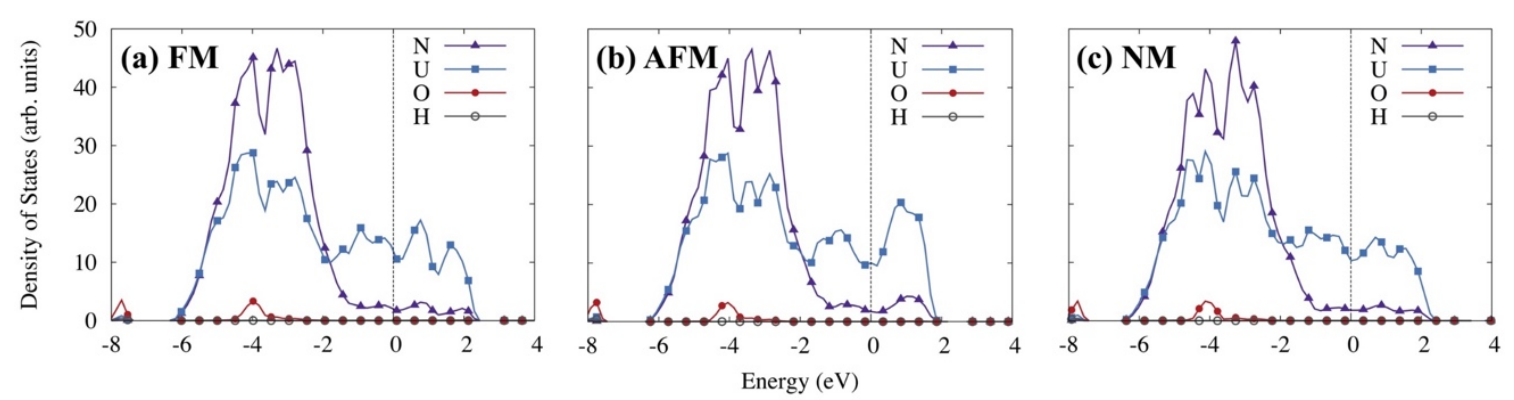

\section{Figure 4.4 Local Densities of States for OH relaxed on (a) FM, (b) AFM, and (c) NM slabs. The Fermi energy is shifted to $0 \mathrm{eV}$.}

Partial Charge Densities (PCD) for $\mathrm{OH}$ adsorbed on the FM, AFM, and NM slabs are shown in Figure 4.5. PCD can project the electronic states of interest onto individual atomic sites, providing further insight into electronic profiles. The electronic states of -6 $\mathrm{eV}$ to the Fermi energy, which capture the majority of the bonding between $\mathrm{U}, \mathrm{N}$, and $\mathrm{O}$ atoms (Figure 4.4), are shown for each magnetic structure in Figure 4.5(a-c). For each structure, valence electrons are localized to $\mathrm{U}, \mathrm{N}$, and the adsorbed $\mathrm{O}$. To better discern variations in bonding across structures, the mapped electronic states are narrowed to the valence electrons from $-2 \mathrm{eV}$ to the Fermi energy in in Figure 4.5(d-f). Across structures, valence electrons are more localized to $\mathrm{U}$ atoms than $\mathrm{N}$ atoms in this energy range. Covalent bonds between the terminating layer of the UN surface and the second layer become weaker in comparison to those in the bulk region. This is evident in the narrower contour regions between $\mathrm{U} 1$ and the rest of the $\mathrm{U}$ and $\mathrm{N}$ atoms. In the FM structure, U1 remains covalently bonded to $\mathrm{N} 1$ and $\mathrm{N} 2$ as well as U3. However, the bond between U1 and $\mathrm{U} 2$ is greatly weakened, shown by the disappearance of the connecting contoured region. In contrast, in AFM U1 remains bonded to N2, U2, and U3, while its bond with N1 is greatly weakened. In NM, while the contoured regions between U1 and its neighboring $\mathrm{N}$ and $\mathrm{U}$ atoms are weakened as compared to the bulk, none of them are weakened to the same extent as in both FM and AFM. 
FM

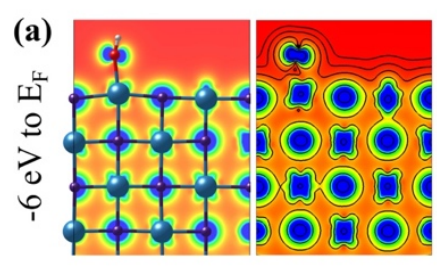

(d)

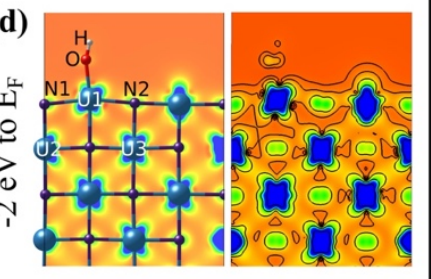

AFM

(b)

(e)

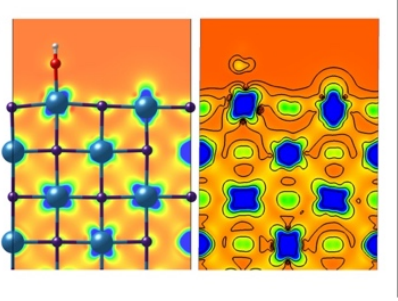

NM

(c)

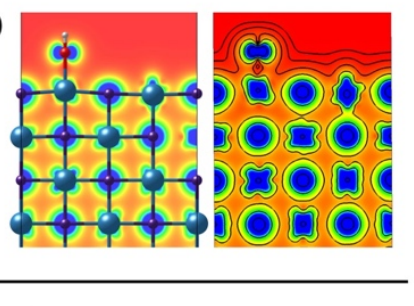

(f)

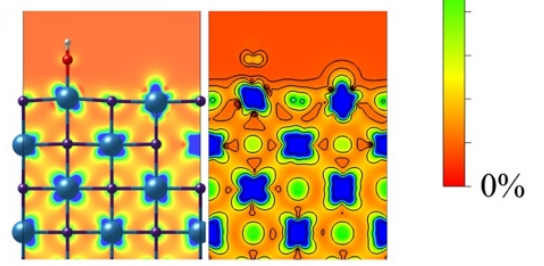

Figure 4.5 Partial Charge Densities for OH adsorbed to FM, AFM, and NM magnetic structures. Valence electrons are shown from (a-c) $-6 \mathrm{eV}$ to the Fermi energy and from (d-f) $-2 \mathrm{eV}$ to the Fermi energy. The scale has been narrowed to $\mathbf{2 5 \%}$ of the total electronic states to improve the visibility of bonds. Contour lines indicate areas with the same energy and are consistent across structures. Blue and red indicate many and no electronic states, respectively. Some $U$ and $N$ atoms have been numbered for reference in the text. The numbering scheme is consistent across magnetic structures.

Electron Localization Function (ELF) analysis can further reveal the overall electron localization, as shown in Figure 4.6. Across structures, the electronic profile around the $\mathrm{OH}$ adsorbate is relatively similar with the majority of charge localized to $\mathrm{H}$ and additional charge below the O. In the FM and NM structures, electrons are equally localized between the $\mathrm{N}$ and $\mathrm{U}$ atoms of the bulk. In contrast, in the AFM structure more electrons are localized to the $U$ atoms with spin-down electrons than the other $U$ atoms and $\mathrm{N}$ atoms. This reveals that $\mathrm{OH}$ adsorption properties on AFM surfaces could vary, depending on if the $\mathrm{OH}$ is adsorbed to a spin-up or spin-down terminated surface. Due to the periodic boundary conditions, both sides of each slab reveal electronic characteristics of the surface. By examining the top of the ELFs, we can compare the electronic profiles of the pristine surfaces without $\mathrm{OH}$ interaction. In the FM structure, the iso-energy contour lines on the pristine surface side reveal a smooth, periodic electronic profile. In 
the AFM structure, there is unphysical charge build-up below the pristine surface, not localized to any atoms. Such charge build-up might occur in slab calculations with insufficient vacuum space to prevent interactions between slab images. However, the lack of such charge in the FM and NM structures suggest the vacuum length is sufficient. This charge build-up in the AFM structure might then be attributed to its metastability. The iso-energy contour lines on the NM pristine surface are not as smooth as that of the FM structure, but there is no unphysical charge localization like the AFM structure.

FM

(a)

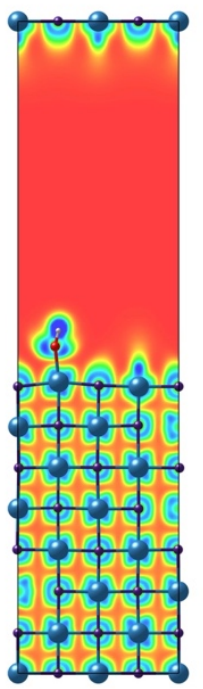

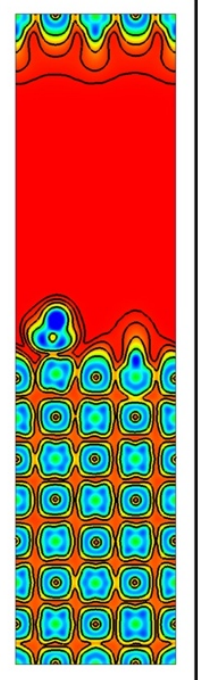

AFM

(b)

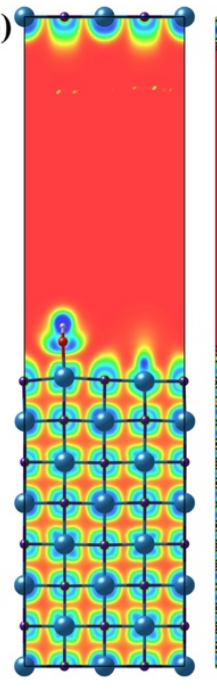

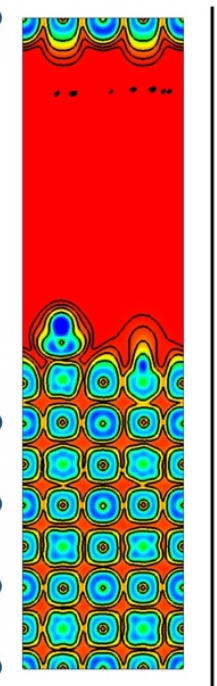

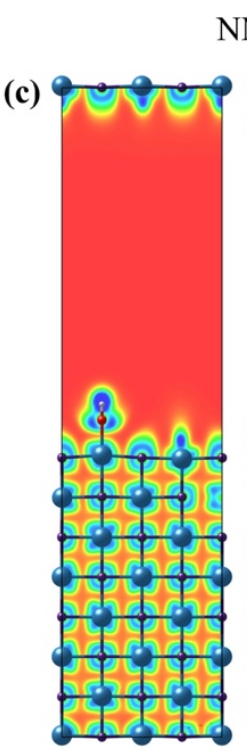

NM

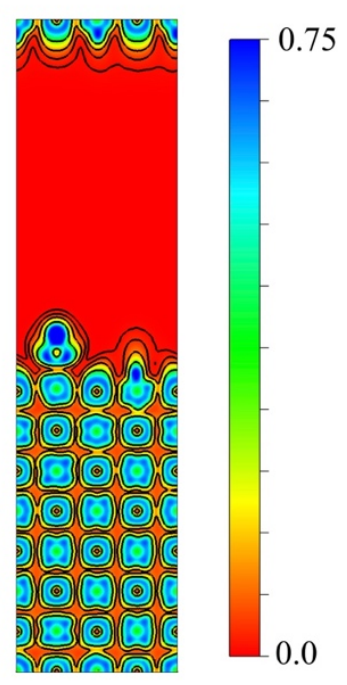

Figure 4.6 Electron Localization Function (ELF) of OH adsorbed to (a) FM, (b) AFM, and (c) NM UN surfaces. Blue and red indicate high and low probability of finding a localized electron, respectively. Contour lines indicate areas with the same energy and are consistent across structures.

\subsection{Discussion}

In bulk UN, a Hubbard U-term of at least $1.65 \mathrm{eV}$ is required to yield AFM as the most stable magnetic treatment [21]. In contrast, we studied FM, AFM, and three HC structures and found FM to be the most stable for the $U_{\text {eff }}$ range of 0.0 to $2.0 \mathrm{eV}$. Thus, no favorable transition between FM and AFM structures was identified. The starting AFM 
structure consisting of alternating spin-up and spin-down (100) planes was the least stable after the U-ramp and revealed an entirely new AFM structure. This structure consists of not only spin-up and spin-down (100) planes, but also planes with half spin-up and halfspin down electrons on $U$ atoms. The magnetic moments of the $U$ atoms in the FM structure ranged from $1.72 \mu_{B}$ in the central layers to $2.10 \mu_{B}$ in the terminating layers. The average magnetic moment of all the $\mathrm{U}$ atoms in the FM structure, $1.88 \mu_{B}$, is in agreement with the $1.89 \mu_{B}$ value found by Claisse et al. for bulk UN [31]. While the magnetic moment determined experimentally is $0.75 \mu_{B}$, Curry et al. theorized this value approaching $3.3 \mu_{B}[13]$.

To determine the effects of magnetic structure on corrosion, $\mathrm{OH}$ was optimized at the U-top site for FM, AFM, and NM magnetic structures. Bond lengths, bond angles, and adsorption energies vary by up to $0.05 \AA, 17.95^{\circ}$, and $0.82 \mathrm{eV}$, respectively, across the three magnetic structures. The LDOS shows similar trends across the magnetic structures including valence electrons localized primarily to $\mathrm{U}$ from -6 to $-2 \mathrm{eV}$ and to $\mathrm{N}$ from $-2 \mathrm{eV}$ to the Fermi energy. Additionally, hybridization of $\mathrm{U}$ and $\mathrm{N}$ with $\mathrm{O}$ occurs around $-4 \mathrm{eV}$. To identify any differences in the electronic profiles when mapped to the atomic structure, PCD was used to map the states from $-6 \mathrm{eV}$ to the Fermi energy and from $-2 \mathrm{eV}$ to the Fermi energy. The PCDs for the $-2 \mathrm{eV}$ to the Fermi energy range reveal variations in bond degradation across magnetic structures. In all three magnetic structures, covalent bonding is weakened between $\mathrm{U} 1$ and its neighboring $\mathrm{U}$ and $\mathrm{N}$ atoms as compared to the bulk. However, in FM the bond between U1 and U2 is weakened to the extent that the contour no longer shows a connecting region. In AFM, the contour no longer shows a connecting region between $\mathrm{U} 1$ and N1. NM does not show bond 
weakening between $\mathrm{U} 1$ and its neighboring atoms to the same extent as FM and AFM. These results suggest that each magnetic structure could suggest different corrosion mechanisms when interacting with dissociated water. Key differences in the ELF can be seen between the AFM structure and the other structures. First, there is an unphysical charge build up next to the pristine surface suggesting metastability. Second, the AFM ELF revealed that more charge is localized to $U$ atoms with spin-down electrons than any other $\mathrm{U}$ or $\mathrm{N}$ atoms. As such, adsorption properties could vary, depending on if the adsorbates interact with a spin-up or spin-down terminated surface.

Our findings reveal insight into the magnetic structures that could be computationally used when studying UN surfaces. While AFM is the most favorable magnetic structure of bulk UN, FM remains the most favorable magnetic structure of the UN surface with consideration of the Hubbard U-term. DFT studies of AFM UN surfaces should consider both slabs terminated with spin-up electrons and spin-down electrons as this can lead to defect energies varying on the order of $0.27 \mathrm{eV}$ (Table 4.2). Additionally, the AFM structure of bulk UN shown in Figure 4.1was not found to be favorable for a surface. As such, future AFM surface studies should employ a metastability treatment to allow the magnetic structure to optimize to a ground state magnetic configuration. Finally, the NM structure was found to yield similar $\mathrm{OH}$ adsorption trends in the LDOS, ELF, and PCD to the FM structure. While the adsorption energy of $\mathrm{OH}$ at the NM slab differed from the FM slab by $0.82 \mathrm{eV}$ (Table 4.2), Ab initio Molecular Dynamics (AIMD) studies ignoring spin can expect to resolve similar electronic profiles to those of DFT. Future computational work studying energetically favorable UN surfaces could reveal more accurate corrosion mechanisms to compare with experiment. 


\section{5 Conclusions}

We performed DFT-based electronic structure calculations and found the FM surface to be the most energetically favorable magnetic structure for the $U_{\text {eff }}$ range of 0.0 to $2.0 \mathrm{eV}$. AFM treatment of alternating (100) spin-up and spin-down planes is not a favorable structure after metastability treatment. It would require a consideration of the effects of spin-up vs. spin-down electron termination on adsorption properties. FM, AFM, and NM treatments yield varying adsorbate bond angles and adsorption energies. The electronic maps of the three magnetic structures indicate similar corrosion mechanisms even though finer resolution of bond weakening in the PCD is seen in FM and AFM. This indicates that future AIMD studies could turn spin consideration off and maintain general electronic profile trends. This evaluation of DFT and AIMD accuracy in adsorption properties supports future opportunities to corroborate computational and experimental UN corrosion studies.

Acknowledgements:

The authors would like to thank the Center for Advanced Energy Studies for supporting this effort, providing access, and enabling collaboration that otherwise may have been unavailable. This research made use of the resources of the High Performance Computing Center at Idaho National Laboratory, which is supported by the Office of Nuclear Energy of the U.S. Department of Energy and the Nuclear Science User Facilities under Contract No.DE-AC07-05ID14517. 


\section{References}

[1] J. Choi, B. Ebbinghaus, T. Meiers, and J. Ahn, Laboratory Directed Research and Development (LDRD) on Mono-Uranium Nitride Fuel Development for SSTAR and Space Applications (Livermore, CA, 2006).

[2] R.M. Dell, V.J. Wheeler, and N.J. Bridger, Trans. Faraday Soc. 63, 1286 (1967).

[3] S. Sugihara and S. Imoto, J. Nucl. Sci. Technol. 6, 237 (1969).

[4] G.A.R. Rao, S.K. Mukerjee, V.N. Vaidya, V. Venugopal, and D.D. Sood, J. Nucl. Mater. 185, 231 (1991).

[5] M. Jolkkonen, P. Malkki, K. Johnson, and J. Wallenius, J. Nucl. Sci. Technol. 54, 513 (2017).

[6] J.K. Watkins, D.P. Butt, and B.J. Jaques, J. Nucl. Mater. 518, 30 (2019).

[7] D. Bocharov, D. Gryaznov, Y.F. Zhukovskii, and E.A. Kotomin, J. Nucl. Mater. 416, 200 (2011).

[8] D. Bocharov, D. Gryaznov, Y.F. Zhukovskii, and E.A. Kotomin, J. Nucl. Mater. 435, 102 (2013).

[9] R.-S. Li, X. Peng, W. Fei, and M. Wen-Yan, Nucl. Sci. Tech. 25, 050502 (2014).

[10] T. Bo, J.-H. Lan, Y.-J. Zhang, Y.-L. Zhao, C.-H. He, Z.-F. Chai, and W.-Q. Shi, Phys. Chem. Chem. Phys. 18, 13255 (2016).

[11] T. Bo, J.-H. Lan, Y.-L. Zhao, C.-H. He, Z.-F. Chai, and W.-Q. Shi, J. Nucl. Mater. 492, 244 (2017).

[12] E.L. Sikorski, T.H. da Silva, L.K. Aagesen, B.J. Jaques, and L. Li, J. Nucl. Mater. 523, 402 (2019).

[13] N.A. Curry, Proc. Phys. Soc. 86, 1193 (1965).

[14] Y.F. Zhukovskii, D. Bocharov, and E.A. Kotomin, J. Nucl. Mater. 393, 504 (2009).

[15] Y.F. Zhukovskii, D. Bocharov, E.A. Kotomin, R.A. Evarestov, and A.V. Bandura, Surf. Sci. 603, 50 (2009).

[16] J. Chen, Z. Long, R. Qiu, Y. Hu, B. Ao, and K. Liu, J. Nucl. Mater. 533, 152095 (2020).

[17] S.L. Dudarev, G.A. Botton, S.Y. Savrasov, Z. Szotek, W.M. Temmerman, and A.P. Sutton, Phys. Status Solidi 166, 429 (1998).

[18] J. Hubbard, Proc. R. Soc. Lond. A. Math. Phys. Sci. 276, 238 (1963). 
[19] J. Hubbard, Proc. R. Soc. Lond. A. Math. Phys. Sci. 285, 542 (1965).

[20] Y. Lu, B.-T. Wang, R.-W. Li, H. Shi, and P. Zhang, J. Nucl. Mater. 406, 218 (2010).

[21] D. Gryaznov, E. Heifets, and E. Kotomin, Phys. Chem. Chem. Phys. 14, 4482 (2012).

[22] D. Bocharov, D. Gryaznov, Y.F. Zhukovskii, and E.A. Kotomin, Surf. Sci. 605, 396 (2011).

[23] D. Rafaja, L. Havela, R. Kužel, F. Wastin, E. Colineau, and T. Gouder, J. Alloys Compd. 386, 87 (2005).

[24] E.L. Bright, R. Springell, D.G. Porter, S.P. Collins, and G.H. Lander, Phys. Rev. B 100, 1 (2019).

[25] P.W. Tasker, J. Phys. C Solid State Phys. 12, 4977 (1979).

[26] G. Kresse and J. Furthmüller, Phys. Rev. B 54, 11169 (1996).

[27] J.P. Perdew, K. Burke, and M. Ernzerhof, 77, 3865 (1996).

[28] B. Dorado, P. Garcia, G. Carlot, C. Davoisne, M. Fraczkiewicz, B. Pasquet, M.

Freyss, C. Valot, G. Baldinozzi, D. Siméone, and M. Bertolus, Phys. Rev. B 83, 035126 (2011).

[29] B. Meredig, A. Thompson, H.A. Hansen, C. Wolverton, and A. Van De Walle, Phys. Rev. B 82, 1 (2010).

[30] R.A. Evarestov, A. V. Bandura, M. V. Losev, E.A. Kotomin, Y.F. Zhukovskii, and D. Bocharov, J. Comput. Chem. 29, 2079 (2008).

[31] A. Claisse, M. Klipfel, N. Lindbom, M. Freyss, and P. Olsson, J. Nucl. Mater. 478, 119 (2016). 


\title{
CHAPTER FIVE: COMBINED EXPERIMENT AND FIRST-PRINCIPLES STUDY OF IN-PILE TEMPERATURE SENSOR MATERIALS
}

\author{
Ember L. Sikorski ${ }^{1,2}$ \\ Richard S. Skifton ${ }^{3}$ \\ Lan $\mathrm{Li}^{1,2}$ \\ ${ }^{1}$ Micron School of Materials Science and Engineering, \\ Boise State University, Boise, ID 83625, USA \\ ${ }^{2}$ Center for Advanced Energy Studies, Idaho Falls, ID 83401, USA \\ ${ }^{3}$ High Temperature Test Laboratory, Idaho National Laboratory, Idaho Falls, ID \\ 83415, USA
}




\begin{abstract}
We leveraged voltage recorded during a heat ramp to tune a computational method to predict the Seebeck electromotive force (EMF) of in-pile temperature sensor materials Molybdenum (Mo) and Niobium ( $\mathrm{Nb}$ ). Using a combined Density Functional Theory (DFT) and Boltzmann Transport Equations (BTE) method, the voltage was predicted but did not include the effects of temperature on atomic structure. Combining Ab Initio Molecular Dynamics (AIMD) and BTE included temperature effects on structure optimization and yielded voltages in a good agreement with experiment. Lanthanum (La) and Phosphorus (P) additives in Mo and Nb, respectively, could increase the EMF compared to those of the pure metals. The presence of $\mathrm{O}$ in Mo increases the EMF while $\mathrm{O}$ in $\mathrm{Nb}$ slightly reduces the EMF. Our studies suggested that heat treatmentinduced structural changes that lead to a reduction in voltage occur not only at the mesoscale as previously understood but also at the atomic scale.
\end{abstract}

\title{
5.1 Introduction
}

Testing accident tolerant fuel (ATF) materials requires subjecting them to higher temperatures than power reactors experience, under normal operation conditions. Research reactors can drive these materials to the temperatures experienced during an accident, allowing for characterization and better understanding of ATF material performance during their namesake scenarios. However, obtaining this characterizing data requires in-pile sensors that can likewise survive under the extreme environment. Thermocouples (TCs) allow for real-time temperature measurement, but the constituent materials must be carefully selected. To meet this need for a TC that can survive both the extreme temperature and irradiation, Idaho National Laboratory (INL) has been 
developing High Temperature Irradiation Resistant Thermocouples (HTIR-TCs)[1-5]. Currently, HTIR-TCs have been shown to endure $1000+$ hours (i.e. $1.2 \times 10^{21}$ neutrons $/ \mathrm{cm}^{2}$ ) at $1247{ }^{\circ} \mathrm{C}$ with no more than $0.6 \%$ drift [5]. At over $1400{ }^{\circ} \mathrm{C}$, HTIR-TCs have endured 2000+ hours with an estimated drift of 2-3\% [6]. Continuing to push the lifetime and accuracy of the HTIR-TC, we seek to better understand possible causes of reduced TC performance or accuracy.

A HTIR-TC consists of two HTIR-thermoelements: Molybdenum (Mo) and Niobium $(\mathrm{Nb})$ wires. They offer the best balance of high temperature and irradiation resistance in comparison with traditional TC thermoelement materials, such as Platinum (Pt), Rhodium (Rh), and Nickel (Ni) alloys [7]. Mo and Nb have thermal nuclear cross sections of 2.65 and 1.15 barns and melting temperatures of 2610 and $2470{ }^{\circ} \mathrm{C}$, respectively [1]. In contrast, Type B (Pt-30\%Rh/Pt-6\%Rh) TCs which are rated for temperatures up to $1820{ }^{\circ} \mathrm{C}$ consist of $\mathrm{Pt}$ and $\mathrm{Rh}$ with thermal cross sections of 10 and 150 barns, respectively. With a large thermal cross section, 6 wt. \% of Rh is expected to transmute during an 800 day irradiation in a pressurized water reactor [8]. TCs composed of Ni alloys: Type K (Chromel/Alumel) and Type N (Nicrosil/Nisil), though less susceptible to irradiation, degrade at temperatures over $1000{ }^{\circ} \mathrm{C}[1,7,9]$. In addition, doping and alloying can further tune Mo for optimal in-pile TC performance. Mo wire doped with Lanthanum Oxide $\left(\mathrm{La}_{2} \mathrm{O}_{3}\right)$ has yielded the highest temperature resolution, or $\mathrm{V} /{ }^{\circ} \mathrm{C}$, allowing for smaller changes in temperature to be reflected in the TC reading [3]. TC performance is controlled by the Seebeck coefficient of each of the TC legs. Utilizing the Seebeck effect, TCs consist of two dissimilar metals connected at a junction as previously shown by Skifton et al. [4]. The temperature difference between the hot 
junction, where temperature is measured in a reactor, and the cold junction, the reference temperature measured outside of the reactor, will produce a proportional voltage difference between the two metals. Once the respective voltage changes have been fitted to the desired temperature range, the TC can be used to monitor temperature.

Mathematically, this Seebeck electromotive force (EMF), E, can be determined by taking the integral of the difference of the Seebeck coefficients for each thermoelement:

$$
E=\int_{T_{r e f}}^{T_{\text {sense }}}\left(S_{+}-S_{-}\right) d T \quad \text { Eq. (5-1) }
$$

where $T_{\text {sense }}$ is the sensing temperature and $T_{r e f}$ is the reference temperature. In a HTIR-TC, the positive Seebeck coefficient, $S_{+}$, corresponds to HTIR-Mo and the negative Seebeck coefficient, $S$-, corresponds to the HTIR-Nb. The Seebeck coefficient is intrinsically controlled by the atomic structure-electronic property relationships of the materials. Given the extreme environment the HTIR-TC is designed for, it is susceptible to structural changes due to temperature, radiation-induced defects, and/or transmutation. These structural changes will affect the Seebeck coefficients of the HTIRthermoelements, resulting in changes in the HTIR-TC performance. By better understanding the structure-property relationships of the thermoelements, we can identify the material factors that enhance and detract from in-pile HTIR-TC performance.

Since the HTIR-TC performance depends on both the atomic and electronic structures of the thermoelements, we leveraged atomistic modeling methods to better understand the controlling factors. In this work, we combined experimental and computational methods to study the effects of heat treatment and thermoelement composition on the HTIR-TC voltage. We investigated the HTIR-Mo and HTIR-Nb thermoelements individually using real-time voltage measurements during heat treatment 
in a tube furnace, Density Functional Theory (DFT), Boltzmann Transport Equations (BTE), and Ab initio Molecular Dynamics (AIMD). First, we examined the effect of heat treatment experimentally on the voltage of each HTIR-thermoelement. Second, through comparing the experimental voltage with the voltage predicted from DFT and BTE we could determine the atomic structure-performance relationships of HTIR thermoelements. Third, we applied AIMD and BTE to determine the temperature effect on the atomic structures and performance of the HTIR thermoelements.

\subsection{Methods}

\subsubsection{Experimental Methods}

Two TCs were fabricated by coupling (1) HTIR-Mo with Pt and (2) HTIR-Nb with Pt. Heat treatment and voltage recording details have been described previously [4,5]. In summary, each thermocouple was placed in a tube furnace opposite a Type B TC, suitable for high temperatures but not irradiation, for temperature measurement. The TCs were heated to $1600{ }^{\circ} \mathrm{C}$ at a rate of $5^{\circ} \mathrm{C} / \mathrm{min}$. The furnace was held at $1600{ }^{\circ} \mathrm{C}$ for $72+$ hours. The furnace was cooled to room temperature at $5^{\circ} \mathrm{C} / \mathrm{min}$. The HTIR-Mo thermoelement contains 0.5 - 1 wt.\% La2O3 and the HTIR-Nb thermoelement contains 496 and $424 \mu \mathrm{g} / \mathrm{g}$ of Phosphorus (P) and Oxygen (O), respectively.

\subsubsection{Computational Methods}

DFT calculations were performed using the Vienna Ab initio Simulation Package (VASP) [10]. Spin-polarized generalized gradient (GGA) exchange-correlation functions were used following the Perdew Burke Ernzerhoff (PBE) formulation [11]. Plane-wave basis sets were implemented utilizing projector-augmented wave (PAW) pseudopotentials. Simulations were performed with a cutoff energy of $550 \mathrm{eV}$ and a 
minimum of $5 \times 5 \times 5$ gamma-centered k-points. The Hubbard $+\mathrm{U}$ term [12] was applied to Mo and $\mathrm{Nb}$ atoms during electronic structure calculations to improve the DFT description of d electrons. Effective U-terms of 8.6 and $4.0 \mathrm{eV}$ were applied to Mo [13] and $\mathrm{Nb}$ [14], respectively. The Boltzmann transport equations were solved using BoltzTraP2 [15].

DFT pure Mo

(a)

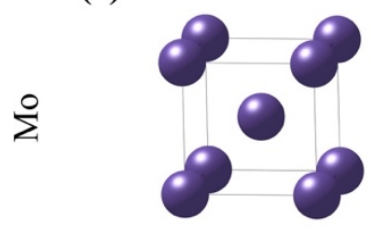

(d)

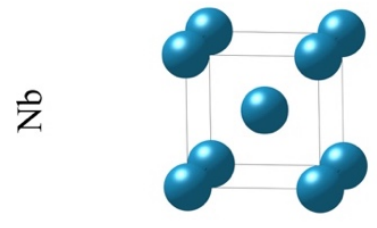

DFT

HTIR-supercell

(b)

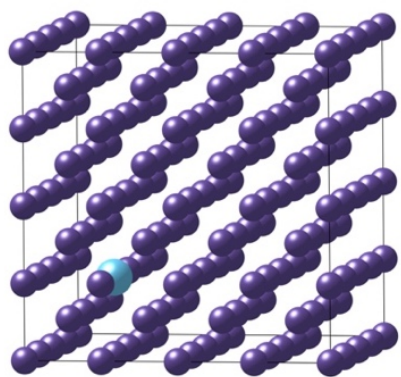

(e)

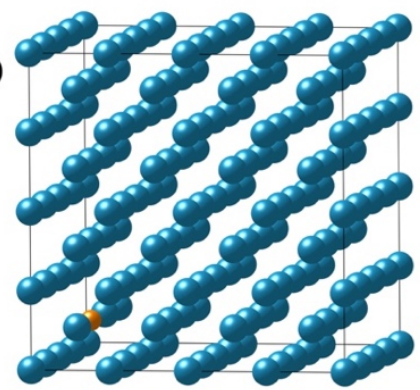

(c)

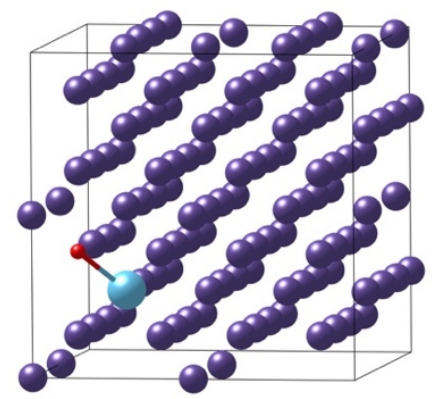

(f)

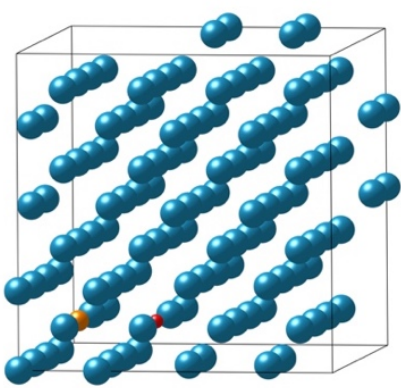

Figure 5.1 Starting atomic structures for (a) pure Mo, (b) the HTIR-Mo supercell used in DFT, (c) the HTIR-Mo supercell used in AIMD, (d) pure Nb, (e) the HTIR-Nb supercell used in DFT, and (f) the HTIR-Nb supercell used in AIMD.

Purple, blue, light blue, orange, and red atoms represent Mo, Nb, La, P, and $\mathrm{O}$, respectively.

For DFT calculations, the pure Mo and $\mathrm{Nb}$ metals were modeled using their Body Center Cubic (BCC) unit cell (Figure 5.1 (a) and (d)) with experimental lattice constants of 3.146 and $3.311 \AA$, respectively $[16,17]$. The Mo unit cell was then supersized to the $4 \times 4 \times 4$ supercell with one atom replaced by La to approximate a concentration of 1.13 wt.\% La. (Figure 5.1 (b)). Similarly, the Nb unit cell was then supersized to the $4 \times 4 \times 4$ 
supercell with one atom replaced by $\mathrm{P}$ to approximate a concentration of $0.26 \mathrm{wt} . \% \mathrm{P}$ (Figure $5.1(\mathrm{e}))$

For AIMD calculations, simulations were performed using an NVT ensemble, 220 $\mathrm{eV}$ cutoff energy, sampled at the gamma point, and with $0.25 \mathrm{fs}$ timesteps. Temperature was controlled with the Nosé-Hoover thermostat and the Nosé mass was set to correspond to 40 time steps $[18,19]$. Convergence tests showed that the $220 \mathrm{eV}$-cutoff structure was converged in comparison with the equivalent equilibration AIMD run at $550 \mathrm{eV}$ as shown in Figure 5.2. In in each system an additional metal atom was replaced with $\mathrm{O}$ to result in Mo 1.13 wt.\% La 0.13 wt.\% O (Figure 5.1 (c)) and $\mathrm{Nb} 0.26$ wt.\% P 0.14 wt.\% O (Figure 5.1 (f)). Electronic structures were obtained for the AIMD-relaxed structures using $320 \mathrm{eV}, 5 \times 5 \times 5$ gamma-centered k-points, and Hubbard $+\mathrm{U}$ terms of 8.6 and $4.0 \mathrm{eV}$ for Mo and $\mathrm{Nb}$, respectively. 


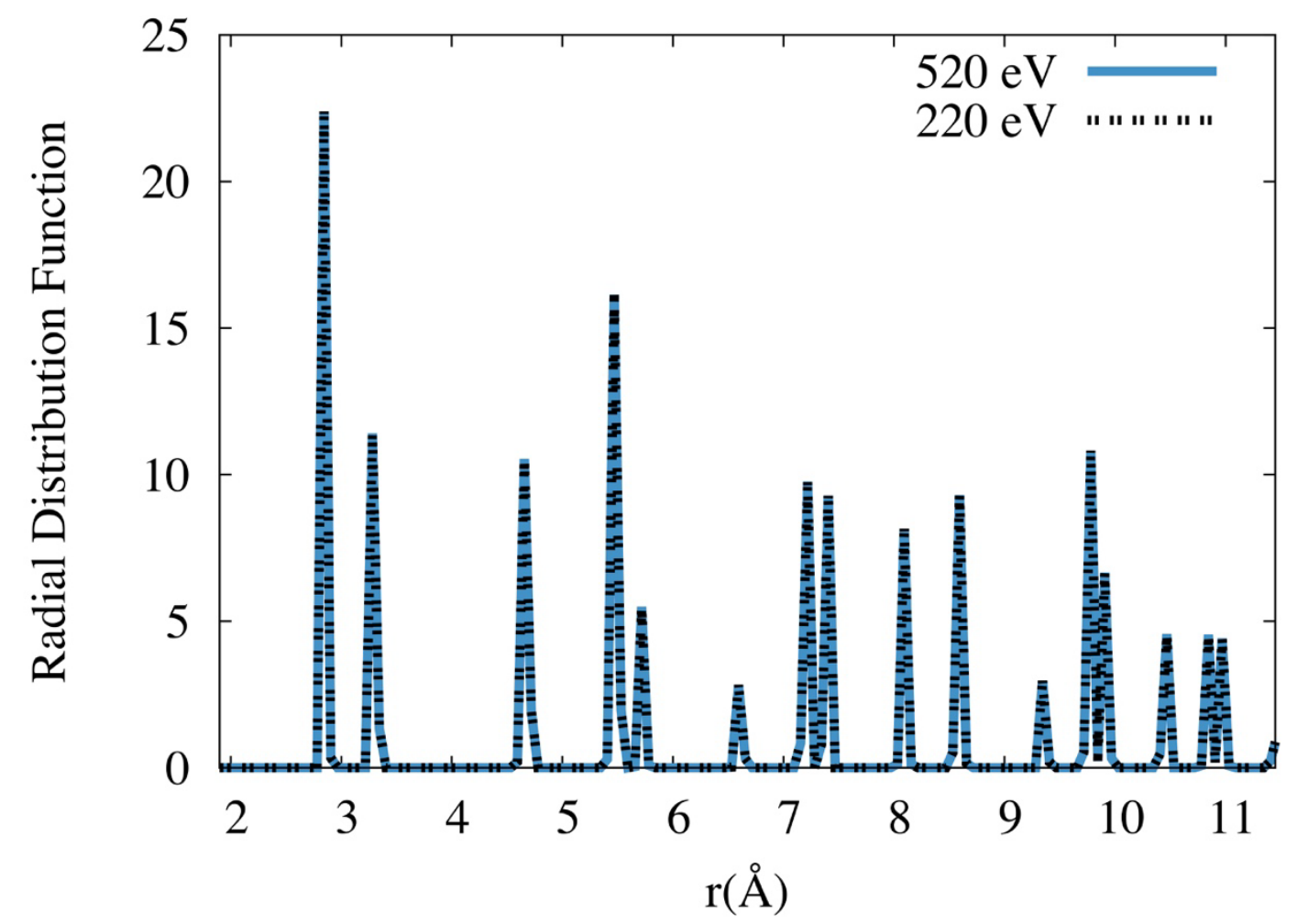

Figure 5.2 Radial Distribution Function in $\AA$ of HTIR-Nb AIMD equilibration at $20{ }^{\circ} \mathrm{C}$ using $220 \mathrm{eV}$ and $520 \mathrm{eV}$ cutoff energies.

\subsection{Results and Discussion}

\subsubsection{Experimental Voltage Measurement}

For in-pile use, the HTIR-TCs are constructed in their now standard HTIR-Mo coupled with HTIR-Nb build. However, the combined voltage of a HTIR-TC is difficult to compare with the absolute, thermoelement specific voltages obtained through modeling. For this reason, we fabricated two special HTIR-TCs where each individual HTIR-thermoelement was coupled with a Pt thermoelement. As the absolute Seebeck voltage of Pt is known experimentally [20], its contribution can be removed to yield the absolute EMF of both HTIR-Mo and HTIR-Nb thermoelements. The results of the experimental heat treatment and voltage measurement are shown in Figure 5.3. Both the HTIR-Mo and HTIR-Nb show variations in the EMF as the temperature approaches 1600 
${ }^{\circ} \mathrm{C}$ and begins the annealing process. In order to better understand the experimental measurements, we applied a combination of DFT, BTE, and AIMD methods to provide insights into the atomic structure and Seebeck coefficient of each thermoelement.

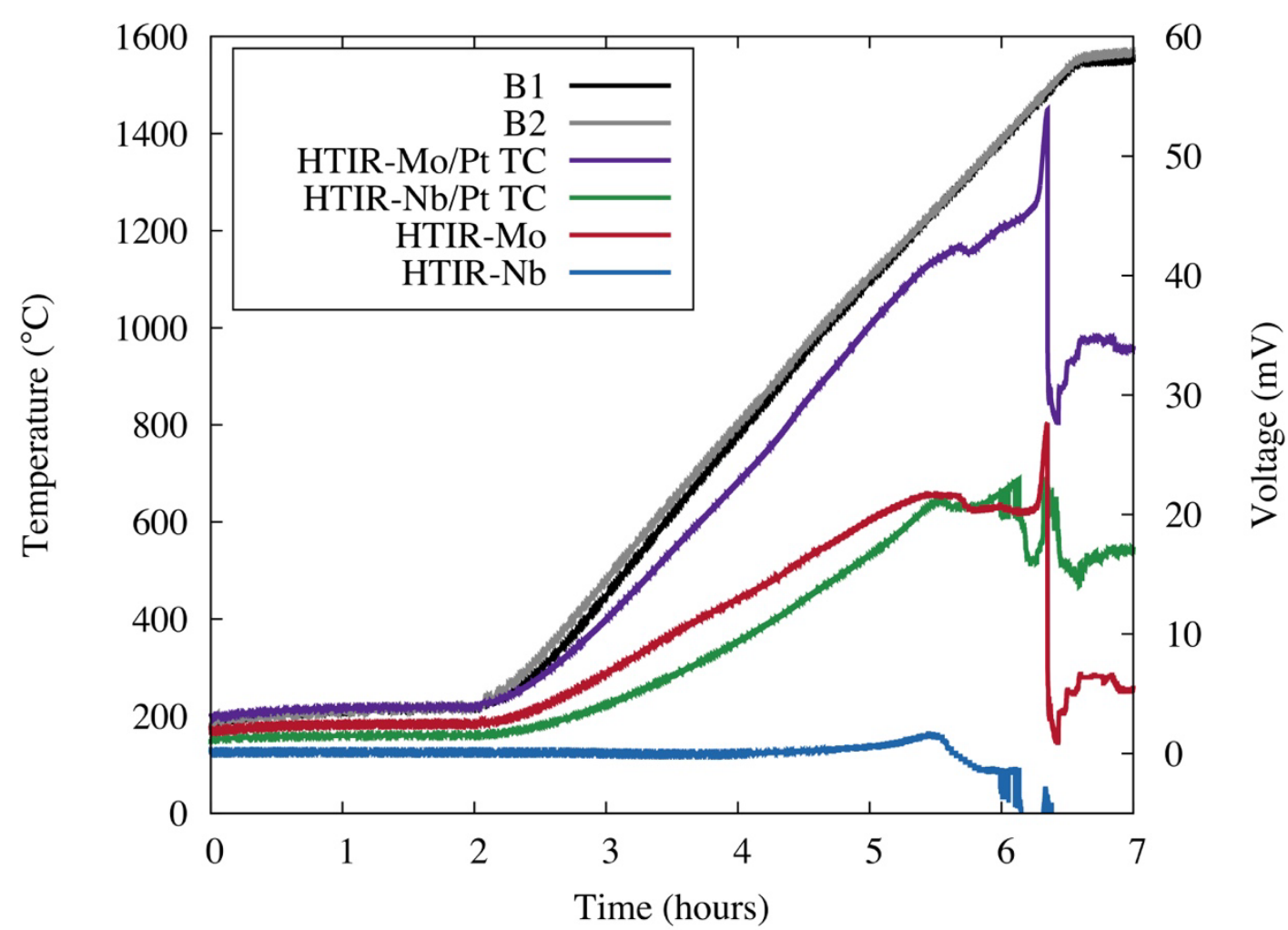

Figure 5.3 Experimental temperature profile as measured by two Type B TCs, voltage output by the HTIR-Nb - Pt and HTIR-Mo - Pt TCs during the heat treatment, and voltage output by the HTIR-Nb and HTIR-Mo thermoelements during the heat treatment. The heat soak at $1600{ }^{\circ} \mathrm{C}$ begins just after 6.5 hours.

\subsubsection{DFT Calculations}

To verify the accuracy of DFT and BTE to predict Seebeck coefficients, we started with pure Mo and pure Nb materials (Figure 5.1 (a) and (d)). Figure 5.4 shows the calculated Seebeck coefficients as a function of temperature, which generally agrees with the experimental values for both pure Mo [21] and pure $\mathrm{Nb}$ [22]. We then extended the models to the supercells containing La and P, as shown in Figure 5.1 (b) and (e), representing HTIR-Mo and HTIR-Nb, respectively. The resultant Seebeck coefficients 
were integrated with respect to temperature as in Eq. (5-1) to obtain the voltage for each thermoelement. Figure 5.5 shows the calculated voltages for the HTIR and pure metals in comparison with the experimental values from Section 5.3.1.

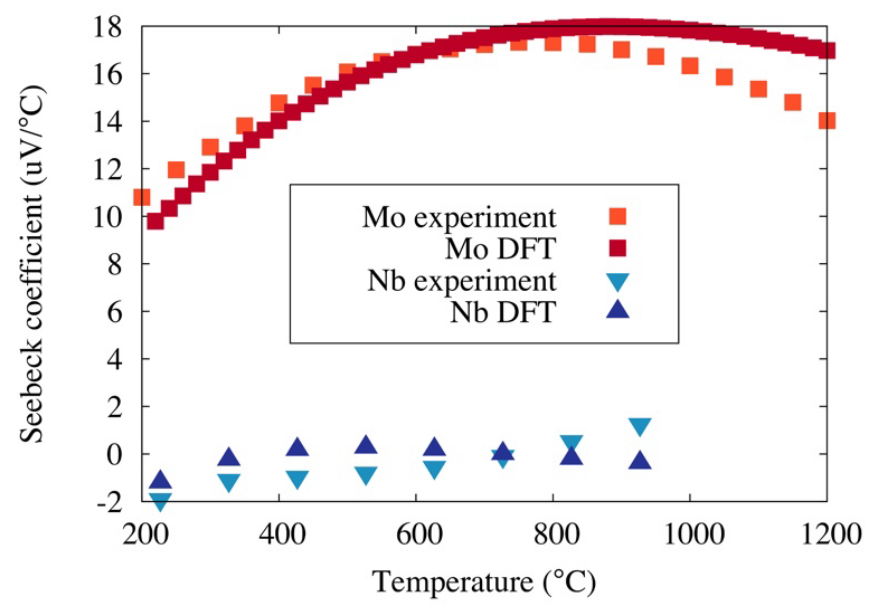

Figure 5.4 Comparison of Seebeck coefficients for pure Mo and Nb from experiment $[21,22]$ and computation.
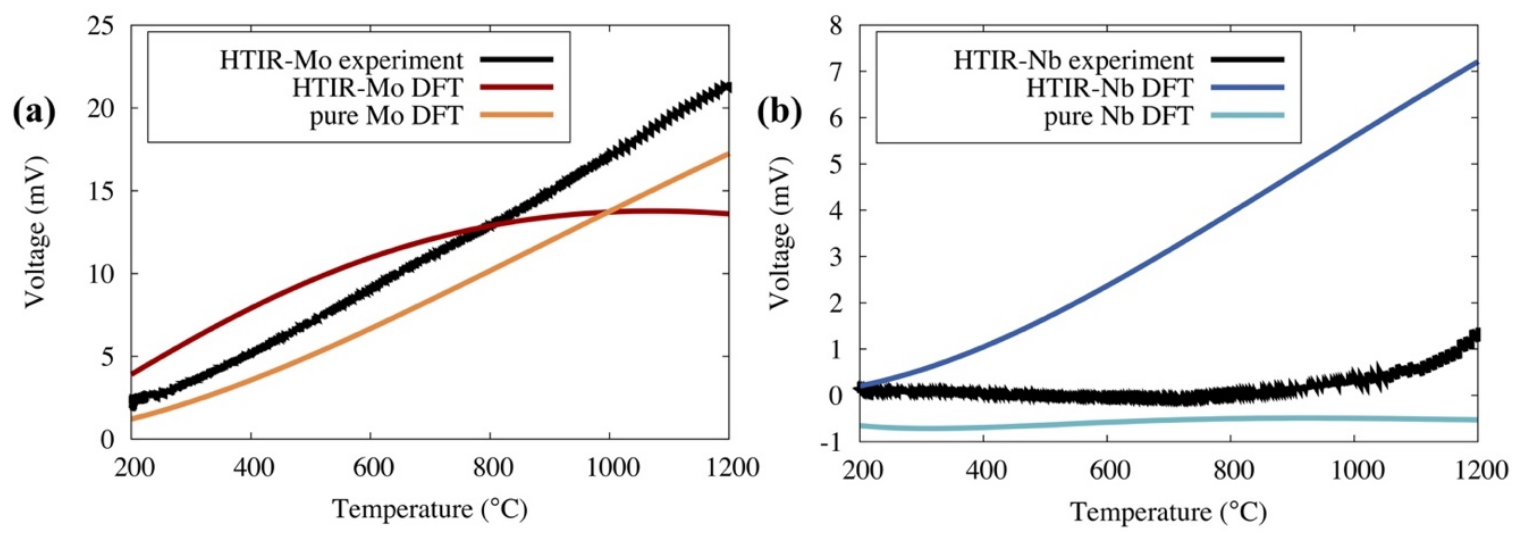

Figure 5.5 Comparison of the voltages obtained from experiment and DFT for (a) Mo and (b) Nb. The HTIR-Mo DFT structure contains 1.13 wt.\% La and the HTIR-Nb DFT structure contains 0.26 wt.\% P.

With the addition of La in Mo, the voltage predicted by DFT-BTE is increased for the majority of the temperature range compared to pure Mo (Figure 5.5 (a)). This voltage increase is in agreement with experimental results demonstrating the high temperature resolution of $\mathrm{Mo}$ with a $\mathrm{La}_{2} \mathrm{O}_{3}$ dopant [3]. The effect of $\mathrm{P}$ on $\mathrm{Nb}$ temperature resolution 
has not previously been reported but is likewise shown here to greatly increase the voltage compared to pure $\mathrm{Nb}$ (Figure 5.5(b)). These results demonstrate a close relationship between atomic structure and Seebeck EMF.

In Figure 5.5(a), there is reasonable agreement between the DFT calculated and experimental HTIR-Mo voltages from 200 to about $600{ }^{\circ} \mathrm{C}$. However, the more parabolic nature of the calculated HTIR-Mo voltage becomes apparent above $600{ }^{\circ} \mathrm{C}$ as it diverges from that of experiment. For HTIR-Nb, the calculated voltage starts off close to the experimental voltage at $200^{\circ} \mathrm{C}$ but diverges as the temperature increases (Figure 5.5 (b)). To understand the implications of these results, we need to examine the assumptions utilized in the DFT-BTE method along with the supercells. First, the atomic structure obtained from DFT was used for the full temperature integration range in the BTE. This assumed that the atomic structure remained the same and no structural distortion would occur at all temperatures, which might be an inaccurate representation especially at higher temperatures. Second, O was not included in the DFT supercells and as such the DFT voltages in Figure 5.5 do not include any contribution from O.

According to Figure 5.5 and DFT assumptions, we can propose four predictions about the HTIR-thermoelements. The variation between the calculated and experimental HTIR-Mo voltage suggests (1) HTIR-Mo undergoes structural changes at or above 600 ${ }^{\circ} \mathrm{C}$, and/or (2) $\mathrm{O}$ affects the trend of the voltage produced by the HTIR-Mo thermoelement. In HTIR-Nb, the addition of P increases the magnitude of the pure $\mathrm{Nb}$ voltage to greater than the experimental voltage. This suggests a competing factor(s), which counteracts the voltage increase from $\mathrm{P}$, ultimately reducing the HTIR-Nb voltage to nearly zero. The competing factor(s) could be (3) structural changes with respect to 
temperature and/or (4) O content. To further validate these predictions, we studied both the HTIR-Mo and the HTIR-Nb system with consideration of the temperature effect using AIMD.

To determine the initial position of $\mathrm{O}$ for AIMD calculations, we performed static calculations of $\mathrm{O}$ at various metal substitutional positions with respect to the La or $\mathrm{P}$ additive in the HTIR supercells. The incorporation energy, $\mathrm{E}_{\text {inc }}$, for $\mathrm{O}$ at each site was calculated using the following equation:

$$
E_{\text {inc }}=E_{n M+A+O}-\left(E_{(n+1) M+A}-E_{M}\right)-\frac{1}{2} E_{O_{2}} \quad, \quad \text { Eq. }(5-2)
$$

where $E_{n M+A+O}$ is the energy of the supercell with $n$ metal atoms containing either the $\mathrm{P}$ or La additive and $\mathrm{O}$ at substitutional metal sites, $E_{(n+1) M+A}$ is the energy of the metal supercell with only a $\mathrm{P}$ or La additive at a substitutional metal site, $E_{M}$ is the energy of a single metal atom in the pure metal unit cell, and $E_{\mathrm{O}_{2}}$ is the energy of a single $\mathrm{O}_{2}$ molecule. Greater magnitude in a positive incorporation energy value indicates more energy required for $\mathrm{O}$ to enter the site. Conversely, greater magnitude in a negative incorporation energy value indicates a more favorable, exothermic process to place $\mathrm{O}$ at the substitutional site. We considered the $1^{\text {st }}$ nearest neighbor site, the $2^{\text {nd }}$ nearest neighbor site, and a site located far from the $\mathrm{P}$ or La additive as substitutional locations for $\mathrm{O}$. As shown in Table 5.1, O can exothermically enter any of the substitutional $\mathrm{Nb}$ sites. However, energy is required to place $\mathrm{O}$ in $\mathrm{Mo}$ at the far or $2 \mathrm{nd}$ nearest neighbor position. Only the $1^{\text {st }}$ nearest neighbor position is exothermically favorable for $\mathrm{O}$ in Mo. The most favorable site for $\mathrm{O}$ in $\mathrm{Nb}$ is the $2^{\text {nd }}$ nearest neighbor with respect to the $\mathrm{P}$ atom while the most favorable site in Mo is the $1^{\text {st }}$ nearest neighbor with respect to the La 
atom. We then started with these most favorable sites for the $\mathrm{O}$ atom in the AIMD

calculations, as shown in Figure 5.1 (c) and (f).

Table 5.1 Incorporation energies of $\mathrm{O}$ in $\mathrm{Nb}$ and Mo when substituting a metal atom far from, at the 1st nearest neighbor, or at the 2nd nearest neighbor site relative to the HTIR additive $P$ or La, respectively.

\begin{tabular}{ll}
\hline \hline O Position & Incorporation Energy $(\mathrm{eV})$ \\
\hline \hline Nb System & \\
\hline Far & -0.50 \\
1st Nearest Neighbor & -0.96 \\
2nd Nearest Neighbor & -1.34 \\
\hline \hline Mo System & \\
\hline Far & 0.65 \\
1st Nearest Neighbor & -0.14 \\
2nd Nearest Neighbor & 0.26 \\
\hline \hline
\end{tabular}

\subsubsection{Ab-Initio Molecular Dynamic Simulations}

Using AIMD allowed us to not only include the effects of temperature on atomic structure but also follow specific heating and cooling profiles. By also including $\mathrm{O}$ in the AIMD simulations, we could evaluate the four hypotheses outlined in the previous section. Experimentally, heat treatment was performed on HTIR-TCs before calibration in order to reduce drift upon a subsequent insertion into a high temperature experiment [4]. Mirroring experimental heat treatment, the AIMD simulations followed the process including the ramp to $1600^{\circ} \mathrm{C}$, the hold at $1600^{\circ} \mathrm{C}$, and the cool down to room temperature. A subsequent ramp was performed to investigate how the treatment stabilized the structure such that subsequent insertion into a reactor yielded less drift than 
it would without heat treatment. The full temperature profile performed using AIMD is shown in Figure 5.6.

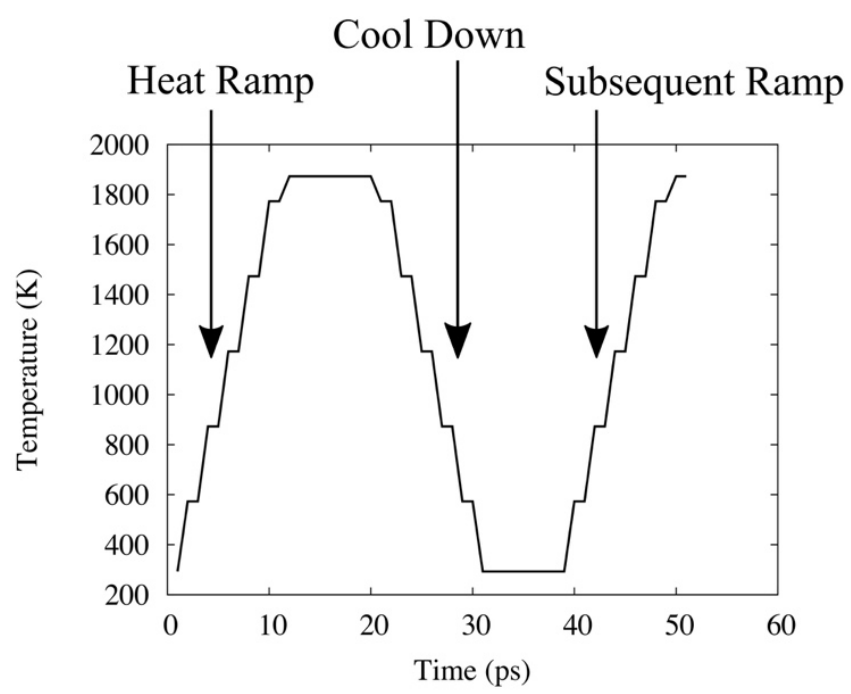

\section{Figure 5.6 Temperature profile of the AIMD heat treatment performed on the HTIR-Mo and HTIR-Nb structures.}

In the DFT-BTE method above, temperature was incorporated only once, during the integration of the Seebeck coefficients. In the AIMD-BTE method, temperature was incorporated twice: first during the simulation of the atomic structure and second during the integration of the Seebeck coefficients. While DFT was performed at 0K, AIMD ran with temperature. By using structures obtained from AIMD as input for BTE, the effects of temperature on atomic structure were included in the calculation of voltage. As shown in Figure 5.7, we can extrapolate each AIMD atomic structures over the full temperature range, like the DFT-BTE method. The AIMD structures utilized for each temperature in Figure 5.7 were taken from the last time step for the corresponding temperature in Figure 5.6 during the initial heat ramp. These computational results are shown against the voltage measured during the experimental heat ramp. 

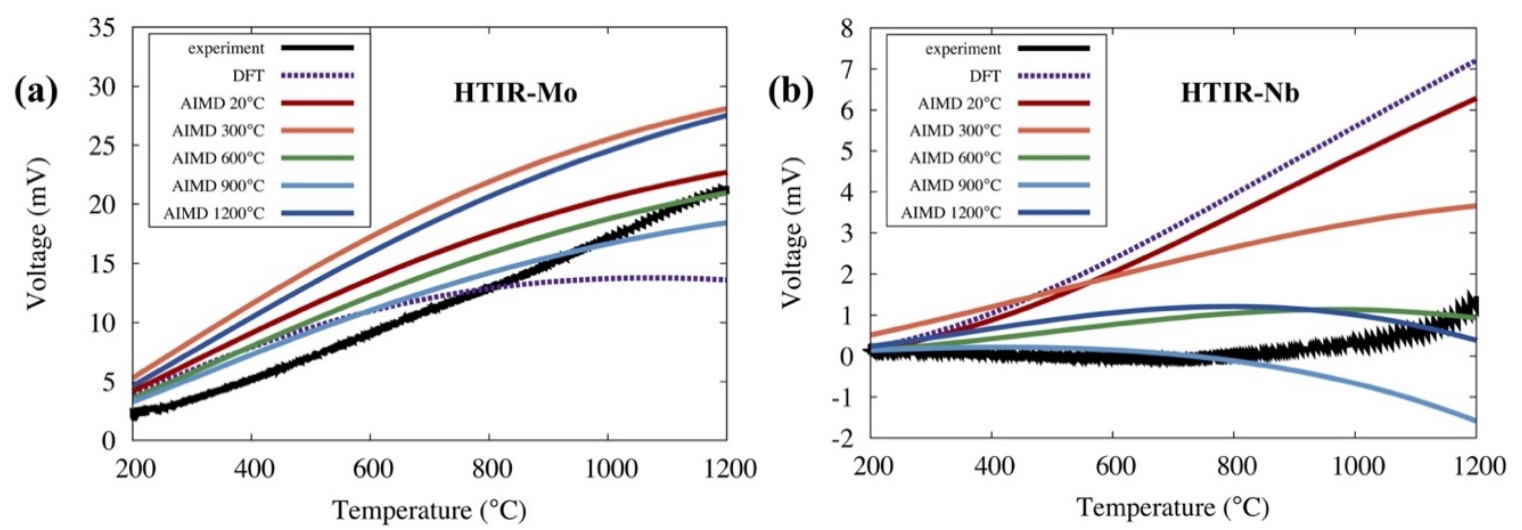

Figure 5.7 Voltage produced by (a) HTIR-Mo and (b) HTIR-Nb as measured in experiment, calculated in DFT, and calculated in AIMD. For the AIMD voltages, Seebeck coefficients obtained from an atomic structure at the given temperature were integrated over the full temperature range. For HTIR-Mo, the DFT structure contains 1.13 wt.\% La and the AIMD structures contain 1.13 wt.\% La 0.13 wt.\% 0. For HTIR-Nb, the DFT structure contains 0.26 wt. $\% \mathrm{P}$ and the AIMD structures contain 0.26 wt.\% P 0.14 wt. $\%$ O.

With the inclusion of $\mathrm{O}$ and temperature effects on the structure, the HTIR-Mo AIMD $20{ }^{\circ} \mathrm{C}$ voltage increases to $25 \mathrm{mV}$ at $1200{ }^{\circ} \mathrm{C}$ (Figure 5.7 (a)), compared to the HTIR-Mo DFT voltage which plateaus at almost $14 \mathrm{mV}$. As the AIMD temperature increases, the HTIR-Mo EMF at $1200^{\circ} \mathrm{C}$ is calculated as $28.1,21.0,18.43$, and $27.5 \mathrm{mV}$ for the $300,600,900$, and $1200^{\circ} \mathrm{C}$ atomic structures, respectively. Experimentally, the HTIR-Mo voltage is $21.37 \mathrm{mV}$ at $1200{ }^{\circ} \mathrm{C}$. The $600^{\circ} \mathrm{C}$ atomic structure gives the closest overall voltage to that measured by experiment.

Like HTIR-Mo, the HTIR-Nb AIMD $20{ }^{\circ} \mathrm{C}$ voltage is the first look at the effects of adding both temperature and $\mathrm{O}$ to the AIMD model (Figure 5.7 (b)). The AIMD $20^{\circ} \mathrm{C}$ voltage is slightly lower than the DFT-BTE curve, yielding voltages of 6.29 and 7.21 $\mathrm{mV}$, respectively, at $1200^{\circ} \mathrm{C}$. As the AIMD temperature increases, the HTIR-Nb EMF at $1200{ }^{\circ} \mathrm{C}$ is calculated as $3.65,0.93,-1.59$, and $0.39 \mathrm{mV}$ for the $300,600,900$, and 1200 ${ }^{\circ} \mathrm{C}$ atomic structures, respectively. Experimentally, the HTIR-Nb voltage is $1.17 \mathrm{mV}$ at $1200{ }^{\circ} \mathrm{C}$. Like HTIR-Mo, the $600{ }^{\circ} \mathrm{C}$ atomic structure gives the closest overall voltage to 
that measured by experiment. As the atomic composition remains constant throughout the heat ramp, the AIMD voltages provide insight into the structural changes with respect to temperature. While DFT overestimates the HTIR-Nb voltage, AIMD demonstrates that structural changes lead to a reduction in the voltage, agreeing better with experiment.

To quantify the structural changes, Radial Distribution Functions (RDFs) (Figure 5.8) were obtained for the HTIR-thermoelements from DFT and from the AIMD heat up/cool down cycles in Figure 5.6. The first peak in an RDF indicates the average distance between each particle and its first nearest neighbor, and so on. The higher and narrower the peak, the more particles that have a neighbor at the given distance. The broader the peak, the more this distance varies from atom to atom. In Figure $5.8(a-b)$, we see the AIMD $20^{\circ} \mathrm{C}$ RDFs are identical to the DFT RDFs for both HTIR-Mo and $\mathrm{Nb}$. This informs us that while both temperature treatment and $\mathrm{O}$ were added during the transition from DFT to AIMD methodology, the only factor contributing to the differences between the DFT voltages and the AIMD $20^{\circ} \mathrm{C}$ voltages (Figure 5.7) is the $\mathrm{O}$ content.

Heating the HTIR-thermoelement systems induces disorder in the structures. In Figure 5.8 (c-f), the RDFs at $20^{\circ} \mathrm{C}$ consist of clearly defined peaks, indicating the crystalline structure expected in the metals. However, starting at $300{ }^{\circ} \mathrm{C}$ the peaks broaden as the temperature increases, indicating a decrease in order. In both the HTIRMo and - $\mathrm{Nb}$ structures during the heat ramp, peak 5 becomes a shoulder of peak 4 , peak 6 and 11 nearly disappear, peaks 7 and 8 merge, and peaks 15 and 16 merge. At $1600{ }^{\circ} \mathrm{C}$ in HTIR-Nb, there are nonzero values between each of the original peaks. This noise is not present to the same extent in the HTIR-Mo system. In HTIR-Nb the noise is so great that 
original peaks 6 and 11 could easily be mistaken for noise. HTIR-Nb exhibits further disorder as original peaks 9 and 10 as well as peaks 14 through 16 merge at $1600{ }^{\circ} \mathrm{C}$. Conversely, in HTIR-Mo peaks 9, 10, and 14 retain definition even though the counts have greatly reduced.

During the cool down shown in Figure 5.8(g-h), nearest neighbor counts begin to return to each of the peaks. The original peaks 4 and 5, peaks 7 and 8, peaks 12 and 13, and peaks 15 and 16 remain merged in both of the structures even after they have returned to room temperature. The intensity of the peaks at $20^{\circ} \mathrm{C}$ after the cool down are significantly reduced compared to the $20{ }^{\circ} \mathrm{C}$ peaks before the heat ramp. As can be seen during the subsequent ramp in Figure $5.8(\mathrm{i}-\mathrm{j})$, this reduces the variation in the peaks between temperatures. Consequently, we can interpret this as less structural change occurring during the subsequent ramp correlating to less drift in the TC measurement. Experimentally, the heat treatment stabilizes the HTIR-thermoelement structures by preemptively causing structural changes. In this way, temperature-induced structural changes are included in the TC calibration, instead of causing drift in performance inpile.

\subsection{Discussion}

In Section 3.2, we outline four predictions about the structural and compositional effects on the voltage produced by each thermoelement. According to the AIMD RDF (Figure 5.8 (c and e)), the HTIR-Mo structure becomes more disordered as the temperature increases. The $600^{\circ} \mathrm{C}$ step exhibits the first complete merge of peaks 7 and 8. The number of peaks and peak intensities remain relatively constant after $600{ }^{\circ} \mathrm{C}$ in agreement with prediction (1). In prediction (2), we hypothesize that $\mathrm{O}$ changes the trend 
of the HTIR-Mo voltage. As shown by the RDFs in Figure 5.8 (a), the DFT and AIMD $20{ }^{\circ} \mathrm{C}$ structures are identical. This indicates that while temperature treatment and $\mathrm{O}$ were added during the transition from DFT to AIMD methodology, the only factor contributing to the difference between the HTIR-Mo DFT voltages and AIMD $20^{\circ} \mathrm{C}$ voltages is the $\mathrm{O}$ content. As such, Figure 5.7(a) shows that $\mathrm{O}$ reduces the parabolic curvature of the voltage. The effect of $\mathrm{O}$ on the HTIR-Mo EMF becomes more pronounced with increasing temperature, i.e. the DFT and AIMD $20{ }^{\circ} \mathrm{C}$ EMFs are less than $0.3 \mathrm{mV}$ apart at $200{ }^{\circ} \mathrm{C}$, but this gap increases to $8.3 \mathrm{mV}$ by $1200{ }^{\circ} \mathrm{C}$. This confirms that O changes the HTIR-Mo voltage trend in agreement with prediction (2).

In prediction (3) we hypothesized that temperature dependent structural changes cancel out the voltage increase due to P. The HTIR-Nb RDFs (Figure 5.8 ( $d$ and f)) show the significant structural changes as temperature increases, with nonzero values between peaks and the merging of even more peaks than in HTIR-Mo. In prediction (4), we hypothesized O could also counteract the voltage increase due to P. Like HTIR-Mo, the HTIR-Nb DFT and AIMD $20^{\circ} \mathrm{C}$ structures are identical (Figure 5.8 (b)). Comparing the DFT and AIMD $20^{\circ} \mathrm{C}$ voltages in Figure 5.7(b) demonstrates that $\mathrm{O}$ does decrease the HTIR-Nb voltage slightly. However, greater voltage reductions are observed as the AIMD temperature increases to $1200^{\circ} \mathrm{C}$. The better agreement of AIMD-BTE than DFTBTE with experiment for HTIR-Nb suggests the DFT-BTE overprediction in voltage is due to the lack of both temperature effects on the structure and O content. Both predictions (3) and (4) are confirmed, though the temperature effects on structure (3) are the larger contributing factor. 
The AIMD-BTE voltage predictions are a considerable improvement over the DFT-BTE predictions, with some AIMD structures, notably $600{ }^{\circ} \mathrm{C}$, in good agreement with experiment over the full temperature range. This suggests the atomic structures obtained at $600{ }^{\circ} \mathrm{C}$ for HTIR-Mo and for HTIR-Nb represent the closest to the average structures of the experimental samples over the full temperature range. This is further supported by the RDFs (Figure 5.8). With the exception of HTIR-Nb at $1600{ }^{\circ} \mathrm{C}$, the number of peaks and peak intensities are largely converged after $600{ }^{\circ} \mathrm{C}$.

Previously, the reduction in voltage of HTIR-TCs during heat treatment was attributed to grain growth [3]. While experiment might have additional structural variations, the RDFs from the AIMD model (Figure 5.8) show the extent to which the heat treatment induces change at length scales as small as atom-atom distances. 

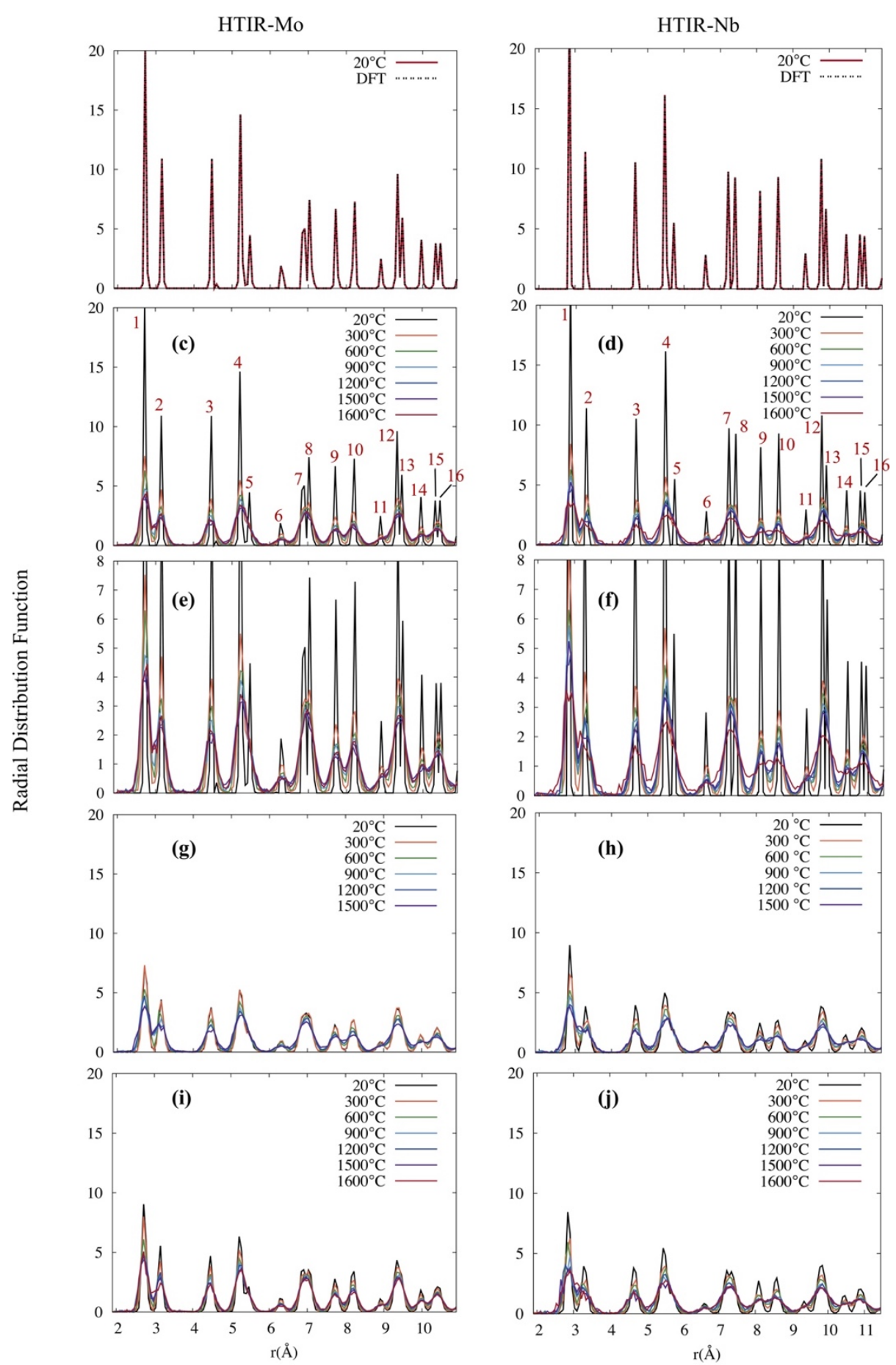

Figure 5.8 Radial Distribution Functions for HTIR-Mo and HTIR-Nb for (a-b) DFT and AIMD $20^{\circ} \mathrm{C}$ and during the AIMD (c-f) heat ramp, (g-h) cool down, and (i-j) subsequent ramp corresponding to the heat up/cool down cycles in Figure 5.6.

The peaks at $20^{\circ} \mathrm{C}$ before the heat ramp are numbered for clarity. A zoomed-in version of the heat ramp is provided (e-f) to better discern the structural evolution. Temperatures indicate the constant temperature simulation at which each RDF was obtained. 


\subsection{Conclusion}

Through a combination of experiment, DFT, BTE, and AIMD, we demonstrated that HTIR-TC performance can be predicted computationally. The combined AIMD and BTE method is the most effective to predict the HTIR-TC structure and Seebeck EMF for the full temperature range. The AIMD structures are converged after $600{ }^{\circ} \mathrm{C}$, resulting in the best voltage match to experiment. The La and $\mathrm{P}$ additives increase the temperature resolution compared to the pure metals, as seen in the DFT-BTE model. In HTIR-Mo, O increases the voltage with increasing temperature. In HTIR-Nb, O causes a slight reduction in the voltage. Temperature significantly affects the atomic structure, leading to changes in the EMF. In both HTIR-Mo and -Nb, the AIMD voltages change over the duration of the heat ramp despite the additive concentrations remaining constant. The decrease in the output voltage during the heat treatment is correlated with the previous study of grain growth. Our further study also shows that structural changes occur at the atomic scale. Our work can support the investigation of different HTIR-TC material builds towards longer lifetimes. The AIMD-BTE method can be used to study the effects of transmutation of the constituent materials, oxidation, or the onset of other radiationinduced point defects on HTIR-TC performance. Additionally, the performance of TC builds consisting of alloyed thermoelements or containing different additives can be predicted. With accurate, durable in-pile temperature sensors, research reactor studies and ATF materials can be better characterized. 
Acknowledgements:

This work was supported in part through the Department of Energy Advanced Sensors and Instrumentation program under DOE Idaho Operations Office Contract DEAC07-05ID14517. The views and opinions of authors expressed herein do not necessarily state or reflect those of the U.S. Government or any agency thereof. Modeling was performed at the High-Performance Computing Center at Idaho National Laboratory, which is supported by the Office of Nuclear Energy of the U.S. Department of Energy and the Nuclear Science User Facilities under Contract No. DE-AC07-05ID14517. Further computational resources were provided by the R2 cluster (DOI: 10.18122/B2S41H) provided by Boise State University's Research Computing Department. 


\section{References}

[1] J.L. Rempe, D.L. Knudson, K.G. Condie, S.C. Wilkins, Thermocouples for HighTemperature In-Pile Testing, Nucl. Technol. 156 (2006) 320-331. doi:10.13182/NT06-A3794.

[2] J.L. Rempe, D.L. Knudson, K.G. Condie, J.C. Crepeau, J.E. Daw, S.C. Wilkins, Options Extending the Applicability of High-Temperature Irradiation-Resistant Thermocouples, Nucl. Technol. 167 (2009) 169-177. doi:10.13182/NT09-14.

[3] J. Daw, J. Crepeau, J. Rempe, D. Knudson, K. Condie, C. Wilkins, Initial Results from Investigations to Enhance the Performance of High Temperature IrradiationResistant Thermocouples, J. Power Energy Syst. 2 (2008) 854-863. doi:10.1299/jpes.2.854.

[4] R. Skifton, J. Palmer, P. Calderoni, Optimization of heat treatment and calibration procedures for high temperature irradiation resistant thermocouples, Instrum. Sci. Technol. 46 (2017) 1-15. doi:10.1080/10739149.2017.1389754.

[5] R.S. Skifton, J. Palmer, L. Kurt, P. Calderoni, D. Corbett, E.L. Sikorski, Summary of High Temperature Irradiation Resistant Thermocouple Standarization Tests, in: 11th Nucl. Plant Instrumentation, Control Human-Machine Interface Technol., 2019.

[6] A.J. Palmer, R.S. Skifton, M. Scervini, D.C. Haggard, W.D. Swank, Performance of Custom-Made Very High Temperature Thermocouples in the Advanced Gas Reactor Experiment AGR-5/6/7 during Irradiation in the Advanced Test Reactor, EPJ Web Conf. ANIMMA 2019. 225 (2020). doi:10.1051/epjconf/202022504010.

[7] B.G. Kim, J.L. Rempe, J. Villard, S. Solstad, Review Paper: Review of Instrumentation for Irradiation Testing of Nuclear Fuels and Materials, Radiat. Meas. Gen. Instrum. 176 (2011) 155-187. doi:10.13182/NT11-A13294.

[8] M. Scervini, C. Rae, B. Lindley, Transmutation of thermocouples in thermal and fast nuclear reactors, in: 2013 3rd Int. Conf. Adv. Nucl. Instrumentation, Meas. Methods Their Appl., 2013. doi:10.1109/ANIMMA.2013.6727900. 
[9] A.J. Palmer, D.C. Haggard, J.W. Herter, M. Scervini, W.D. Swank, D.L. Knudson, R.S. Cherry, Summary of thermocouple performance during advanced gas reactor fuel irradiation experiments in the advanced test reactor and out-ofpile thermocouple testing in support of such experiments, in: 2015 4th Int. Conf. Adv. Nucl. Instrumentation, Meas. Methods Their Appl., 2015. doi:10.1109/ANIMMA.2015.7465501.

[10] G. Kresse, J. Furthmüller, Efficient iterative schemes for ab initio total-energy calculations using a plane-wave basis set, Phys. Rev. B. 54 (1996) 11169-11186. doi:10.1103/PhysRevB.54.11169.

[11] J.P. Perdew, K. Burke, M. Ernzerhof, Generalized Gradient Approximation Made Simple, 77 (1996) 3865-3868. doi:doi.org/10.1103/PhysRevLett.77.3865.

[12] S.L. Dudarev, G.A. Botton, S.Y. Savrasov, Z. Szotek, W.M. Temmerman, A.P. Sutton, Electronic Structure and Elastic Properties of Strongly Correlated Metal Oxides from First Principles: LSDA + U, SIC-LSDA and EELS Study of UO2 and NiO, Phys. Status Solidi. 166 (1998) 429-443. doi:10.1002/(SICI)1521396X(199803)166:1<429::AID-PSSA429>3.0.CO;2-F.

[13] S. Lutfalla, V. Shapovalov, A.T. Bell, Calibration of the DFT/GGA+U Method for Determination of Reduction Energies for Transition and Rare Earth Metal Oxides of Ti, V, Mo, and Ce, J. Chem. Theory Comput. 7 (2011) 2218-2223. doi:10.1021/ct200202g.

[14] H. Kamisaka, T. Suenaga, H. Nakamura, K. Yamashita, DFT-Based Theoretical Calculations of Nb-and W-Doped Anatase TiO 2: Complex Formation between W Dopants and Oxygen Vacancies, J. Phys. Chem. C. 114 (2010) 12777-12783. doi:10.1021/jp104355q.

[15] G.K.H. Madsen, J. Carrete, M.J. Verstraete, BoltzTraP2, a program for interpolating band structures and calculating semi-classical transport coefficients, Comput. Phys. Commun. 231 (2018) 140-145. doi:10.1016/j.cpc.2018.05.010. 
[16] C. Bernuy-Lopez, M. Allix, C.A. Bridges, J.B. Claridge, M.J. Rosseinsky, Sr2MgMoO6- $\delta$ : Structure, phase stability, and cation site order control of reduction, Chem. Mater. 19 (2007) 1035-1043. doi:10.1021/cm0624116.

[17] A.U. Seybolt, Solid Solubility of Oxygen In Columbium, JOM. 6 (1954) 774776. doi:10.1007/BF03377426.

[18] N. Cusack, P. Kendall, The absolute scale of thermoelectric power at high temperature, Proc. Phys. Soc. 72 (1958) 898-901. doi:10.1088/03701328/72/5/429.

[19] R. Schley, G. Metauer, Thermocouples for measurements under conditions of high temperature and nuclear radiation, Temp. Its Meas. Control Sci. Ind. 5 (1982) 1109-1113.

[20] V. Raag, H. V Kowger, Thermoelectric Properties of Niobium in the Temperature Range $300^{\circ}-1200^{\circ}$ K, J. Appl. Phys. 36 (1965) 2045-2048. doi:10.1063/1.322341. 


\title{
CHAPTER SIX: OXIDATION AND CORROSION OF IN-PILE TEMPERATURE SENSOR MATERIALS
}

\author{
Ember L. Sikorski ${ }^{1,2}$ \\ Richard S. Skifton ${ }^{3}$ \\ Lan $\mathrm{Li}^{1,2}$ \\ ${ }^{1}$ Micron School of Materials Science and Engineering, \\ Boise State University, Boise, ID 83625, USA \\ ${ }^{2}$ Center for Advanced Energy Studies, Idaho Falls, ID 83401, USA \\ ${ }^{3}$ High Temperature Test Laboratory, Idaho National Laboratory, Idaho Falls, ID \\ 83415, USA
}




\begin{abstract}
High Temperature Irradiation Resistant Thermocouples (HTIR-TCs) are used to obtain real-time temperature data from research reactors. HTIR-TCs must withstand corrosion, oxidation, and hydrogen embrittlement caused by the reactor coolant. Previous experimental studies of HTIR thermoelements Mo and Nb have focused on oxidation in the 550 to $1100{ }^{\circ} \mathrm{C}$ range. In this work, we used $\mathrm{Ab}$ initio Molecular Dynamics (AIMD) to investigate $\mathrm{Mo}$ and $\mathrm{Nb}$ alloys exposed to oxygen and water from 20 to $1600{ }^{\circ} \mathrm{C}$. Mo$1 \mathrm{wt} . \% \mathrm{Nb}$ is predicted to have the best overall resistance to both oxidation and corrosion of all the studied thermoelement materials. Of the studied $\mathrm{Nb}$ alloys, $\mathrm{Nb}-1 \mathrm{wt} . \% \mathrm{Zr}$ has the best oxidation resistance from 20 to $600{ }^{\circ} \mathrm{C}$, and $\mathrm{Nb}-1 \mathrm{wt} . \% \mathrm{Mo}$ has the best oxidation resistance from 600 to $1600{ }^{\circ} \mathrm{C}$. $\mathrm{Nb}-1 \mathrm{wt} . \%$ Mo has the best corrosion resistance of the studied $\mathrm{Nb}$ alloys.
\end{abstract}

\title{
6.1 Introduction
}

Advanced nuclear materials are continually being developed to improve accident tolerance, reactor lifetime, efficiency, and economic viability [1-4]. Both accidents and advanced nuclear reactors drive materials to temperatures well beyond the normal operating condition of the current reactor fleet. During normal operation Light Water Reactors operate at around $300{ }^{\circ} \mathrm{C}$, but a loss of coolant accident can drive temperatures to $1500{ }^{\circ} \mathrm{C}[5]$. To evaluate how nuclear materials would respond to higher temperatures, testing is conducted in research reactors ranging from 900 to $1500{ }^{\circ} \mathrm{C}$, with some programs seeking temperatures up to $1800{ }^{\circ} \mathrm{C}[1,6]$. In order to get real-time data of the temperature profiles during research reactor experiments, robust sensors are needed that can withstand the temperature as well as irradiation. For this reason, Idaho National 
Laboratory has been developing High Temperature Irradiation Resistant Thermocouples (HTIR-TCs).

HTIR-TCs have been able to sustain $1500^{\circ} \mathrm{C}$ readings for up to 85 days, outperforming the next leading nuclear grade TC by $300^{\circ} \mathrm{C}[7]$. To prevent drift, HTIRTCs are heat treated above their desired operating temperature, usually at $1600{ }^{\circ} \mathrm{C}$. To extend the lifetime of HTIR-TCs, a better understanding is needed of their reactivity with research reactor coolant. Research reactors such as the Advanced Test Reactor (ATR) and the Transient Reactor Test Facility (TREAT) are water-cooled and air-cooled, respectively. As such, HTIR-TCs need to endure inherently corrosive and oxidizing environments.

HTIR-TCs consist of a HTIR-Mo and HTIR-Nb thermoelement. These HTIR thermoelement compositions have been carefully selected to optimize temperature resolution and ductility while minimizing grain growth $[6,8,9]$. Towards improving temperature resolution and radiation resistance, $\mathrm{Mo}-\mathrm{Nb}$ alloys are considered. $\mathrm{Zr}$ additions to $\mathrm{Nb}$ could delay grain growth, so $\mathrm{Nb}-1 \% \mathrm{Zr}$ has been used as a HTIR-TC sheathing thermoelement materials. Depending on the HTIR-TC build, one of thermoelements may be exposed to the coolant. Previous experimental studies have investigated the oxidation processes of pure $\mathrm{Nb}$ ranging from 600 to $1100{ }^{\circ} \mathrm{C}$ [10-12] and pure Mo from 550 to $1700^{\circ} \mathrm{C}$ [13]. Corrosion studies including Mo and $\mathrm{Nb}$ have focused on the role of each metal as an additive. However, the performance of Mo alloyed with $\mathrm{Nb}$ or vice versa in oxidizing or corrosive environments remains unknown.

First-principles modeling methods have been used rigorously in nuclear systems to determine physical properties and reaction mechanisms. Density Functional Theory 
(DFT) allows for the precise selection of reactants on an atomistic scale, which can be leveraged to understand material performance when exposed to coolant. Similarly, DFT can be used to investigate candidate materials for which experimental data is limited. To investigate atomistic properties and include temperature effects, researchers have also performed Ab initio Molecular Dynamics (AIMD). With the key role of temperature in nuclear energy studies, this method has been widely applied to study point defects in $\gamma$-U [14], Xe behavior in UN [15], and SiC corrosion [16,17].

AIMD can help us to extend the investigation of Mo-Nb alloys with a greater temperature range, compare reactivity with $\mathrm{O}_{2}$ as well as $\mathrm{H}_{2} \mathrm{O}$, and study the behavior of developmental HTIR alloys.

In this study, we focused on three candidate alloys: $\mathrm{Nb}-1 \% \mathrm{Zr}$, Mo- $1 \% \mathrm{Nb}$, and $\mathrm{Nb}-1 \% \mathrm{Mo}$ in comparison with pure Mo and $\mathrm{Nb}$. Each surface was studied under an $\mathrm{H}_{2} \mathrm{O}$ and $\mathrm{O}_{2}$ atmosphere from 20 to $1600{ }^{\circ} \mathrm{C}$. Specifically, we first evaluated atomic mechanisms, by which the coolant reacted with the pure metal or alloy surfaces. We then used electronic mapping to understand the process of the reaction and the effect of the corrosion or oxidation on the bonding characteristics of the pure metals and alloys. The diffusion coefficients for each surface were compared to ascertain which is most resistant to reactor coolant.

\subsection{Methods}

AIMD and DFT calculations were performed using the Vienna Ab initio Simulation Package (VASP) [18]. Spin-polarized generalized gradient (GGA) exchangecorrelation functional was used following the Perdew Burke Ernzerhoff (PBE) formulation [19]. Plane-wave basis sets were implemented utilizing projector-augmented 
wave (PAW) pseudopotentials. AIMD simulations were performed using an NVT ensemble with a cutoff energy of $220 \mathrm{eV}$ and sampled at the gamma point with $0.5 \mathrm{fs}$ timesteps. Temperature was considered using the Nosé-Hoover thermostat. The Nosé mass was set to 40 timesteps.

Electron Localization Function (ELF) [20] and Partial Charge Density (PCD) calculations of the AIMD relaxed structures were performed with a cutoff energy of 600 $\mathrm{eV}$ and $8 \times 8 \times 2$ gamma-centered k-points. The Hubbard $+\mathrm{U}$ term [21] was applied to Mo and $\mathrm{Nb}$ atoms during these electronic structure calculations to improve the DFT description of d electrons. Effective U-terms of 8.6 and $4.0 \mathrm{eV}$ were applied to Mo [22] and $\mathrm{Nb}$ [23], respectively. Climbing-image nudged elastic band (NEB) with 6 images was used to calculate activation energies [24].

Metal slabs were constructed using $3 \times 3 \times 4$ of their respective unit cells resulting in 8 monolayers and a surface area of 9 atoms. Our test calculations showed total energy for the $3 \times 3 \times 4$ to be converged with the $4 \times 4 \times 4$ to $0.05 \mathrm{eV} /$ atom. Our previous work showed adsorption energies were sensitive up to 8 monolayers [25]. The bottom two layers of each surface were fixed during structure optimization to represent the bulk. To form $\mathrm{Nb}-1 \% \mathrm{Zr}, \mathrm{Nb}-1 \% \mathrm{Mo}$, and $\mathrm{Mo}-1 \% \mathrm{Nb}$, one majority atom in the terminating layer was replaced with $\mathrm{Zr}, \mathrm{Mo}$, or $\mathrm{Nb}$, respectively, resulting in $\mathrm{Nb}-1.36 \mathrm{wt} \% \mathrm{Zr}, \mathrm{Nb}-$ 1.43wt.\%Mo, and Mo-1.35wt.\%Nb. 18 adsorbate molecules, either $\mathrm{O}_{2}$ or $\mathrm{H}_{2} \mathrm{O}$, were applied to each slab to yield 2 monolayer coverage, i.e. 2 molecules to 1 metal surface atom, to accelerate the reactions [26]. Simulations were performed 20, 300, 600, 1000, 1300 , and $1600{ }^{\circ} \mathrm{C}$ to encompass the temperatures at which HTIR-TCs are approved for 
use. The higher temperatures can also accelerate the diffusion such that the mechanisms are observable within the AIMD time scale [16,26,27].

\subsection{Results}

Oxidation and corrosion due to research reactor coolant may contribute to failure in HTIR-TCs. To better understand the extent to which HTIR thermoelements may oxidize or corrode and if a developmental alloy may be more resistant, $\mathrm{Nb}, \mathrm{Nb}-1 \mathrm{wt} . \% \mathrm{Zr}$, Nb-1wt.\%Mo, Mo, and Mo-1wt.\%Nb were studied. Each surface was subjected to two monolayers of $\mathrm{O}_{2}$ and $\mathrm{H}_{2} \mathrm{O}$ separately and heated at 20,300, 600, 1000, 1300, and 1600 ${ }^{\circ} \mathrm{C}$ using AIMD. Each surface, adsorbate, and temperature combination were simulated for $10 \mathrm{ps}$. To calculate the diffusion coefficients, the first $4 \mathrm{ps}$ of each run were considered as equilibration time and the mean squared displacement of the adsorbates was taken over the remaining 6 ps. The diffusion coefficient pre-factor and activation energy obtained from the equation of fit for each surface allows us to compare the extend of oxidation or corrosion we may expect for each material.

\subsubsection{Interaction with $\mathrm{O}_{2}$}

The $\mathrm{O}$ diffusion coefficients for the surfaces subjected to $\mathrm{O}_{2}$ are shown in Figure 6.1. The corresponding activation energies and diffusion coefficient pre-factors found from the trendlines are shown in Table 6.1. The activation barrier for $\mathrm{O}$ diffusion into the surface is lowest for $\mathrm{Nb}-1 \% \mathrm{Mo}$, followed by Mo- $1 \% \mathrm{Nb}, \mathrm{Nb}, \mathrm{Mo}$, and $\mathrm{Nb}-1 \% \mathrm{Zr}$. The diffusion coefficient pre-factors from least to greatest are $\mathrm{Mo}-1 \% \mathrm{Nb}, \mathrm{Nb}-1 \% \mathrm{Mo}$, Mo, $\mathrm{Nb}-1 \% \mathrm{Zr}$, and $\mathrm{Nb}$. 


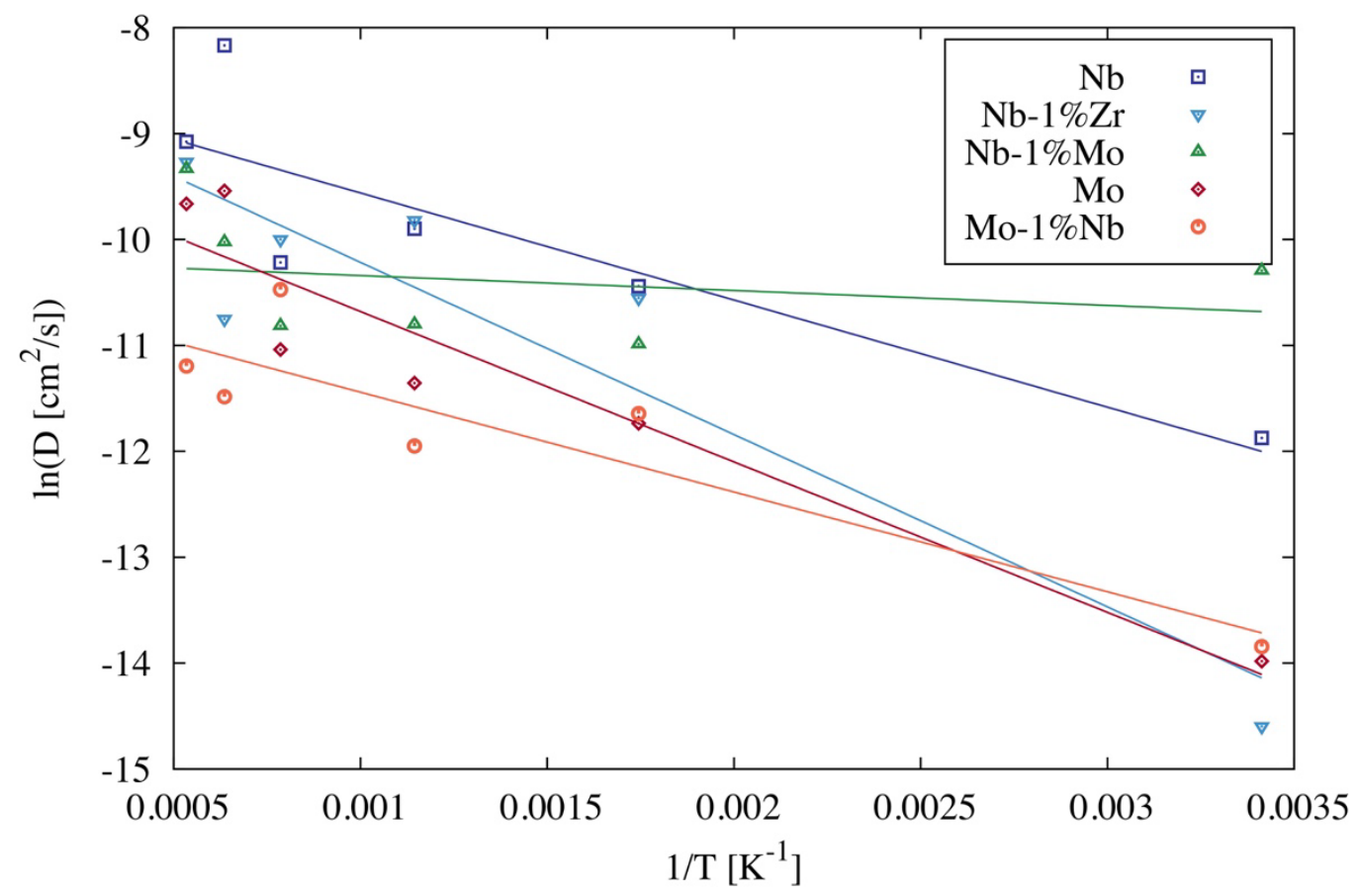

Figure 6.1 Diffusion coefficients of each surface exposed to $\mathrm{O2}$.

Table 6.1 Diffusion coefficient pre-factor and activation energy for $\mathbf{O}$ at each surface.

\begin{tabular}{llll}
\hline & $\mathrm{D} 0\left[\mathrm{~cm}^{\wedge} 2 / \mathrm{s}\right]$ & $\mathrm{EA}[\mathrm{eV}]$ & $\mathrm{EA}[\mathrm{kJ} / \mathrm{mol}]$ \\
\hline $\mathrm{Nb}$ & $2.01 \mathrm{E}-04$ & 0.087 & 8.405 \\
$\mathrm{Nb}-1 \% \mathrm{Zr}$ & $1.86 \mathrm{E}-04$ & 0.140 & 13.519 \\
$\mathrm{Nb}-1 \% \mathrm{Mo}$ & $3.72 \mathrm{E}-05$ & 0.012 & 1.172 \\
$\mathrm{Mo}$ & $9.52 \mathrm{E}-05$ & 0.122 & 11.806 \\
$\mathrm{Mo}-1 \% \mathrm{Nb}$ & $2.75 \mathrm{E}-05$ & 0.081 & 7.832 \\
\hline
\end{tabular}


To better understand how $\mathrm{O}_{2}$ causes structural changes to each surface, the atomic structures after $10 \mathrm{ps}$ at the highest temperature $1600{ }^{\circ} \mathrm{C}$, which is expected to accelerate the reactions, are shown in Figure 6.2. At the $\mathrm{Nb}$ surface, $\mathrm{O}$ is able to intercalate into the $3^{\text {rd }}$ layer from the surface. The terminating $\mathrm{Nb}$ atoms are pulled away from their original positions as the oxide layer forms. The similar scenario occurs in $\mathrm{Nb}-1 \% \mathrm{Zr}$ and $\mathrm{Nb}-$ $1 \% \mathrm{Mo} . \mathrm{Zr}$ is the most favorable atom $\mathrm{O}$ prefers to bond with, followed by $\mathrm{Nb}$ and then Mo. At the predominantly Mo surfaces, $\mathrm{O}$ can only intercalate up to the $2^{\text {nd }}$ layer from the original surface termination. $\mathrm{Nb}$ is more favorable for $\mathrm{O}$ to bond with than Mo.

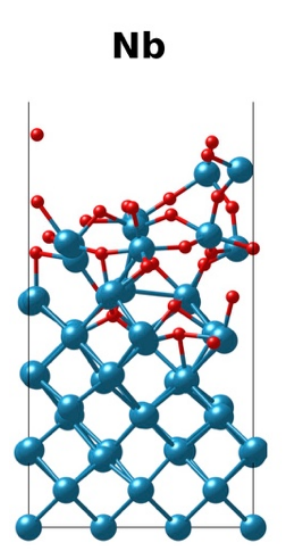

Figure 6.2
Nb-1\%Zr

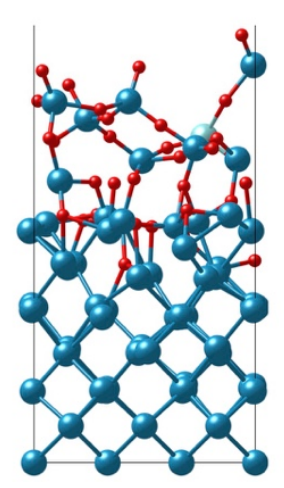

Nb-1\%Mo

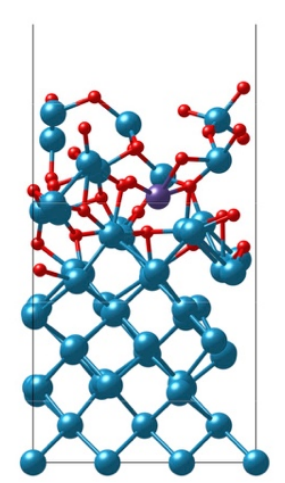

Mo

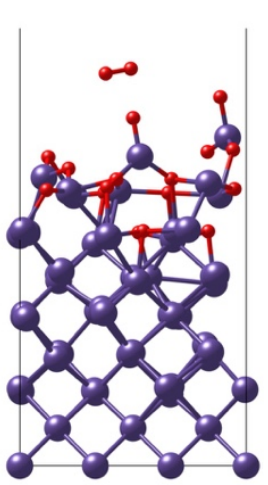

Mo-1\%Nb

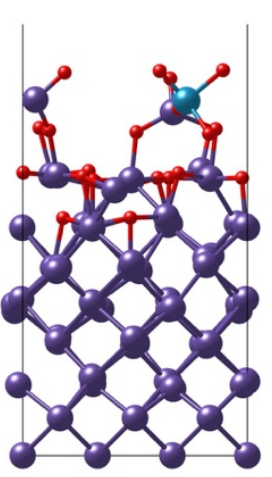

Atomic structures of metals interacting with $\mathrm{O2}$ after $10 \mathrm{ps}$ at 1600 ${ }^{\circ} \mathrm{C}$. Blue, purple, teal, and red atoms represent $\mathrm{Nb}, \mathrm{Mo}, \mathrm{Zr}$, and $\mathrm{O}$, respectively.

To resolve the electronic profiles of the valence electrons, PCD was mapped to each system exposed to O2. By adding contour lines to PCD maps, the same energy regions can reveal bonding. Atoms with significant valence electron localization are likely to continue reacting. By comparing the bonding between atoms in the bulk to that of the terminating layer, the extent of structural degradation can be evaluated. To investigate the electrons most likely to take part in reactions, the charge profiles of the valence electrons resolved from $-2 \mathrm{eV}$ to the Fermi level as the $0.5 \mathrm{ps}$ and 1 ps timesteps for each $\mathrm{O} 2$ system are shown in Figure 6.3. At $0.5 \mathrm{ps}$ in pure $\mathrm{Nb}$, charge is localized 
primarily to the $\mathrm{O} 2$ molecules not bonded with the surface. The $\mathrm{O}$ that intercalated into the surface shows a local reduction in charge. After $1 \mathrm{ps}$, the intercalated $\mathrm{O}$ has more charge localized to it. One $\mathrm{Nb}$ atom is displaced even further away from the surface. The $\mathrm{Nb}$ atoms in the oxide layer maintain as much charge localization as the $\mathrm{Nb}$ atoms representing the bulk. This suggests that the oxide layer still has valence electrons available to support further oxide growth.

At $0.5 \mathrm{ps}$ in the $\mathrm{Nb}-1 \mathrm{Zr}$ surface, charge is predominantly localized to the surface and the $\mathrm{O} 2$. At $1 \mathrm{ps}$, charge relocates to the $\mathrm{O}$ atoms bonded to the $\mathrm{Zr}$. Unlike at the pure $\mathrm{Nb}$ surface, little charge is localized to the $\mathrm{Nb}$ atoms in the newly formed oxide layer. Thus, less electrons may be available to take part in further oxide formation.

At $0.5 \mathrm{ps}$ for the $\mathrm{Nb}-1$ Mo surface, charge is localized to the $\mathrm{O} 2$, the intercalated $\mathrm{O}$, and the bulk $\mathrm{Nb}$ atoms. Less charge is localized to the Mo atom as less contour lines connect it to the bulk metallic bonds. However, as the simulation progresses to $1 \mathrm{ps}$ the yellow and green metallic bonds extend to the Mo.

In the predominantly Mo surfaces, very little charge is localized to the Mo atoms. The $\mathrm{O}$ atoms appear to be able to maintain more charge in their original $\mathrm{O}_{2}$ form than by bonding to the surface. As the Mo systems progress to $1 \mathrm{ps}$, nearly as much charge relocates to the Mo bulk layers as the adsorbed $\mathrm{O}$. At $1 \mathrm{ps}$ at the Mo-1Nb surface, charge is predominantly localized to $\mathrm{O}_{2}$ followed by $\mathrm{O}$ bonded with $\mathrm{Nb}$.

To examine all of the calculated electrons, the ELF method was used. The ELF shows the structural integrity with consideration of all electrons rather than a select energy range. The ELF can also show where charge is predominantly localized overall. The ELF in Figure 6.4 maps all the calculated electrons for each surface exposed to $\mathrm{O}_{2}$. 
For $\mathrm{Nb}$, more electrons are localized to the $\mathrm{O}_{2}$ and the $\mathrm{O}$ atoms in the oxide layer. $\mathrm{The} \mathrm{Nb}$ atoms in the oxide layer are no longer completely surrounded radially by metallic bonds. For $\mathrm{Nb}-1 \mathrm{Zr}$, a charge void is shown in the oxide layer as was seen in the PCD (Figure 6.3). For $\mathrm{Nb}-1 \mathrm{Mo}$, more charge is localized to the $\mathrm{O}$ atom bonded with $\mathrm{Nb}$ atoms than the $\mathrm{O}$ atom bonded to Mo. The Mo and furthest intercalated $\mathrm{O}$ atom have nearly no charge localized to them. The Mo surface shows the metallic bonds and generally more electrons localized to the unabsorbed $\mathrm{O}_{2}$ molecules than the adsorbed $\mathrm{O}$ atoms. In Mo-1Nb, more electrons are also localized to the $\mathrm{O}_{2}$ molecule. While $\mathrm{O}$ atoms adsorb to the surface, additional charge appears to localize into the deeper Mo bulk layers. 


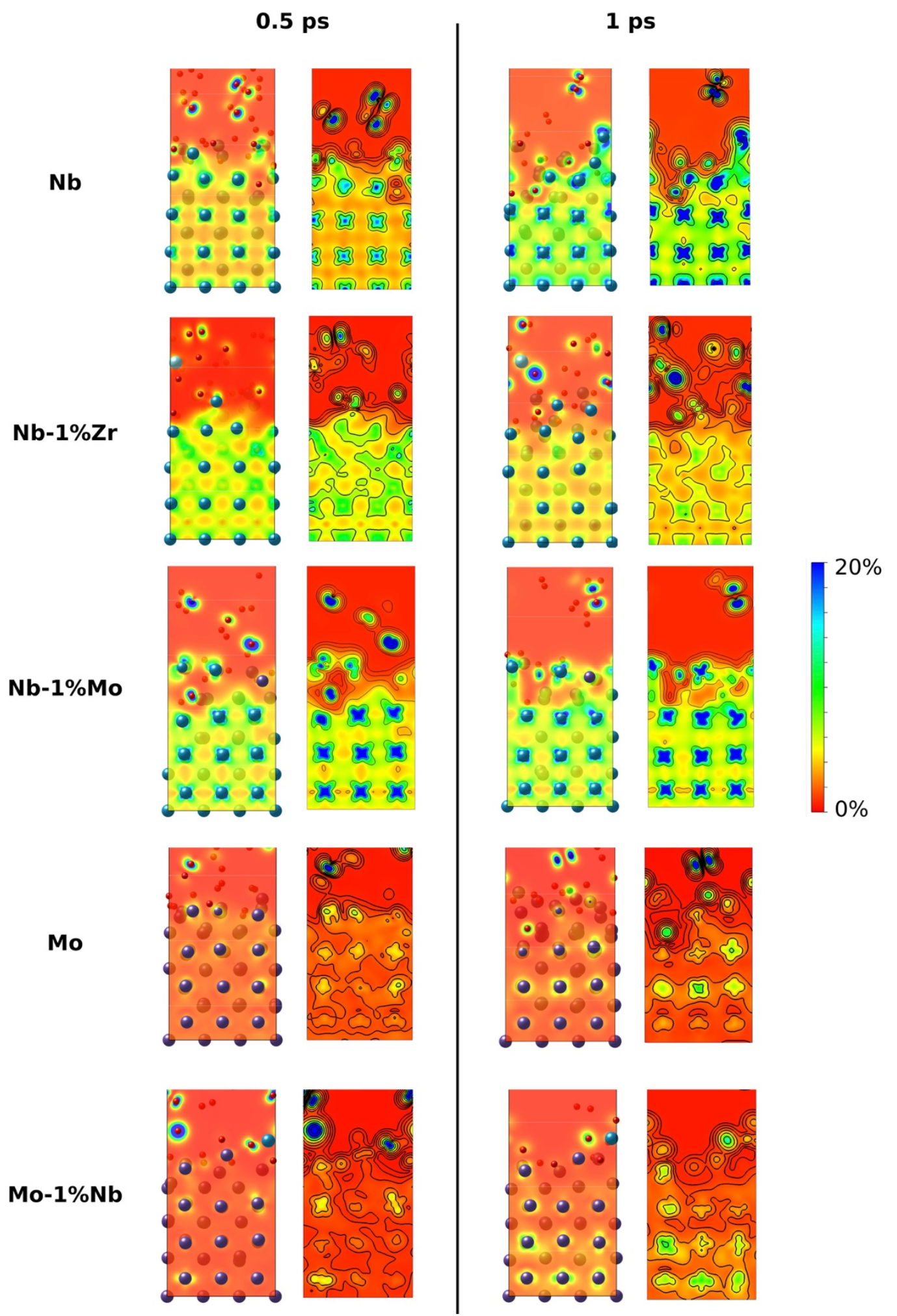

Figure 6.3 Partial Charge Densities of metal surfaces under 02 after 0.5 and 1 ps at $1600{ }^{\circ} \mathrm{C}$. Electrons are resolved from $-2 \mathrm{eV}$ to the Fermi level. The scale has been narrowed to $20 \%$ of the total electronic states to improve the visibility of bonds.

Contour lines indicate areas with the same energy and are consistent across structures. Blue and red indicate many and no electronic states, respectively. 
Nb

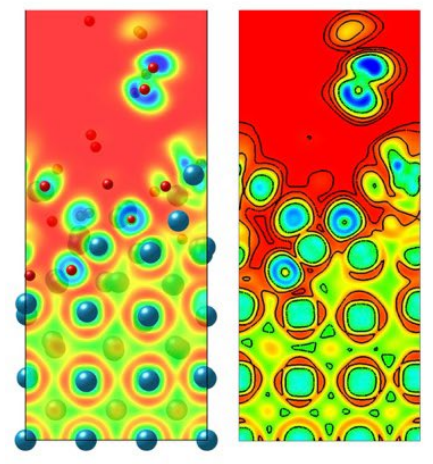

$\mathrm{Nb}-1 \% \mathrm{Zr}$
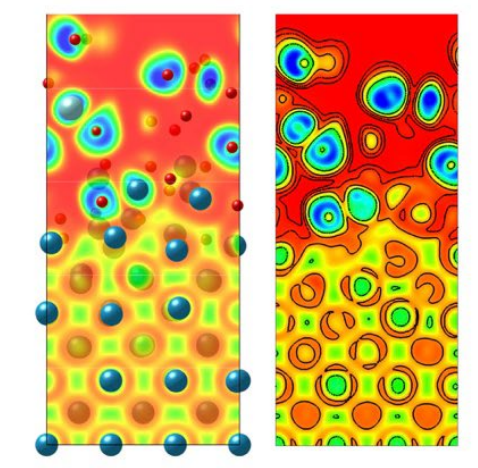

Nb-1\%Mo
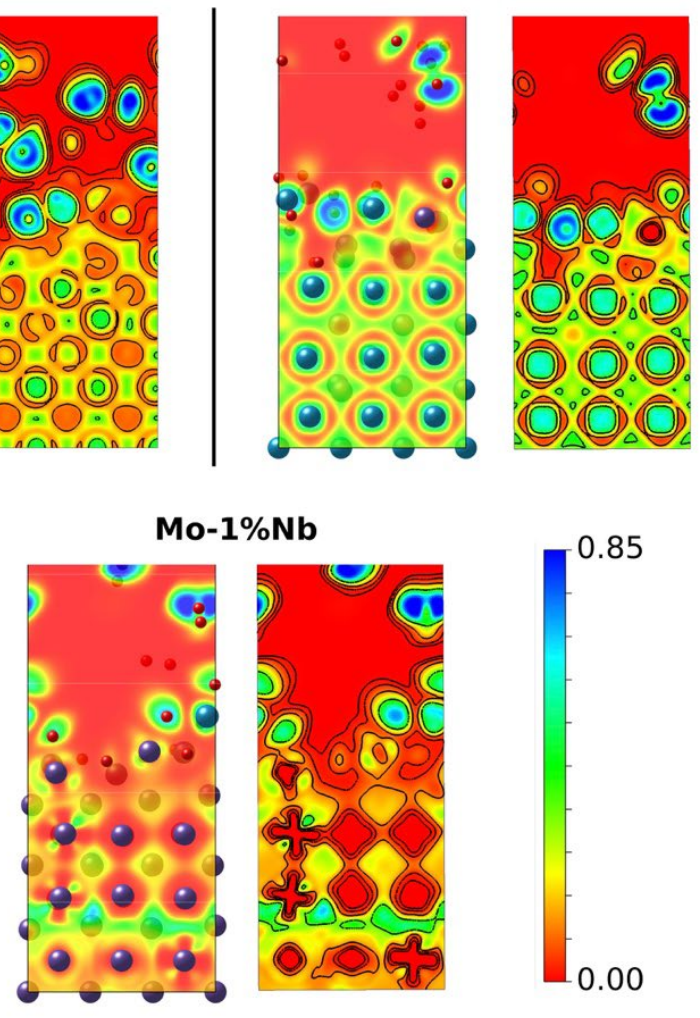

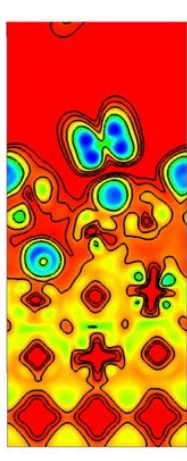

Figure 6.4 Electron Localization Functions of metal surfaces under $\mathrm{O}_{2}$ after $1 \mathrm{ps}$
at $1600{ }^{\circ} \mathrm{C}$. Electrons are resolved from $-2 \mathrm{eV}$ to the Fermi level. Contour lines indicate areas with the same energy and are consistent across structures. Blue and red indicate a high and low probability of finding an electron, respectively.

\subsubsection{Interaction with $\mathrm{H}_{2} \underline{\mathrm{O}}$}

The diffusion coefficients for the surfaces subjected to $\mathrm{H}_{2} \mathrm{O}$ are shown in Figure

6.5. As the $\mathrm{O}$ and $\mathrm{H}$ atoms have quite different diffusion mechanisms in these systems, the diffusion coefficients are split into those for $\mathrm{O}$ and $\mathrm{H}$ atoms separately. The $\mathrm{H}_{2} \mathrm{O}$ diffusion coefficient pre-factors and activation energies are given in Table 6.2. The activation barrier for $\mathrm{O}$ diffusion from $\mathrm{H}_{2} \mathrm{O}$ into the surface is lowest for $\mathrm{Mo}-1 \% \mathrm{Nb}$, followed by $\mathrm{Mo}, \mathrm{Nb}, \mathrm{Nb}-1 \% \mathrm{Zr}$, and $\mathrm{Nb}-1 \% \mathrm{Mo}$. The $\mathrm{O}$ diffusion coefficient pre-factors from least to greatest are $\mathrm{Mo}-1 \% \mathrm{Nb}, \mathrm{Mo}, \mathrm{Nb}-1 \% \mathrm{Zr}, \mathrm{Nb}$, and $\mathrm{Nb}-1 \% \mathrm{Mo}$. For $\mathrm{H}$ diffusion from $\mathrm{H}_{2} \mathrm{O}$ in the surface, the lowest activation barrier is $\mathrm{Mo}$, followed by $\mathrm{Mo}-1 \% \mathrm{Nb}, \mathrm{Nb}$, $\mathrm{Nb}-1 \% \mathrm{Zr}$, and $\mathrm{Nb}-1 \% \mathrm{Mo}$. The $\mathrm{H}$ diffusion coefficient pre-factors from least to greatest 
are $\mathrm{Mo}, \mathrm{Mo}-1 \% \mathrm{Nb}, \mathrm{Nb}-1 \% \mathrm{Mo}, \mathrm{Nb}-1 \% \mathrm{Zr}$, and $\mathrm{Nb}$. The atomic structures for each surface exposed to $\mathrm{H}_{2} \mathrm{O}$ after $10 \mathrm{ps}$ at $1600{ }^{\circ} \mathrm{C}$ are shown in Figure 6.6. $\mathrm{H}_{2} \mathrm{O}$ dissociates into primarily adsorbed $\mathrm{O}$ and absorbed $\mathrm{H}$, with some adsorbed $\mathrm{OH}$. $\mathrm{H}$ can reach the $7^{\text {th }}$ layer from the $\mathrm{Nb}$ surface, the $6^{\text {th }}$ layer from the $\mathrm{Nb}-1 \% \mathrm{Zr}$ surface, and the $5^{\text {th }}$ layer from the $\mathrm{Nb}-1 \%$ Mo surface. $\mathrm{H}$ remains at the terminating layer for both of the predominantly Mo surfaces. $\mathrm{O}$ atoms preferentially bond to the $\mathrm{Zr}$, followed by $\mathrm{Nb}$, and lastly Mo. While the $\mathrm{O}$ and $\mathrm{H}$ activation energies from $\mathrm{H}_{2} \mathrm{O}$ are lower for the predominantly Mo surfaces, the $\mathrm{O}$ and $\mathrm{H}$ atoms remain at the terminating layer. Thus, these activation energies reflect adsorption energy on the surface rather than an energy barrier to intercalate into the surface.

To determine a more accurate activation energy to $\mathrm{O}$ intercalation into the Mo surface, NEB calculations were performed. O can adsorb to the Mo surface at either a Mo-top or hollow site with adsorption energies of -6.39 and $-6.76 \mathrm{eV}$, respectively. NEB calculations could predict the transition energy from both adsorption sites to an interstitial site as shown in Figure 6.7. The most likely intercalation pathway is from the hollow site to the interstitial site via pathway II with an activation energy of $3.08 \mathrm{eV}$ as opposed to $78.25 \mathrm{eV}$ for pathway I from the top site to the interstitial.
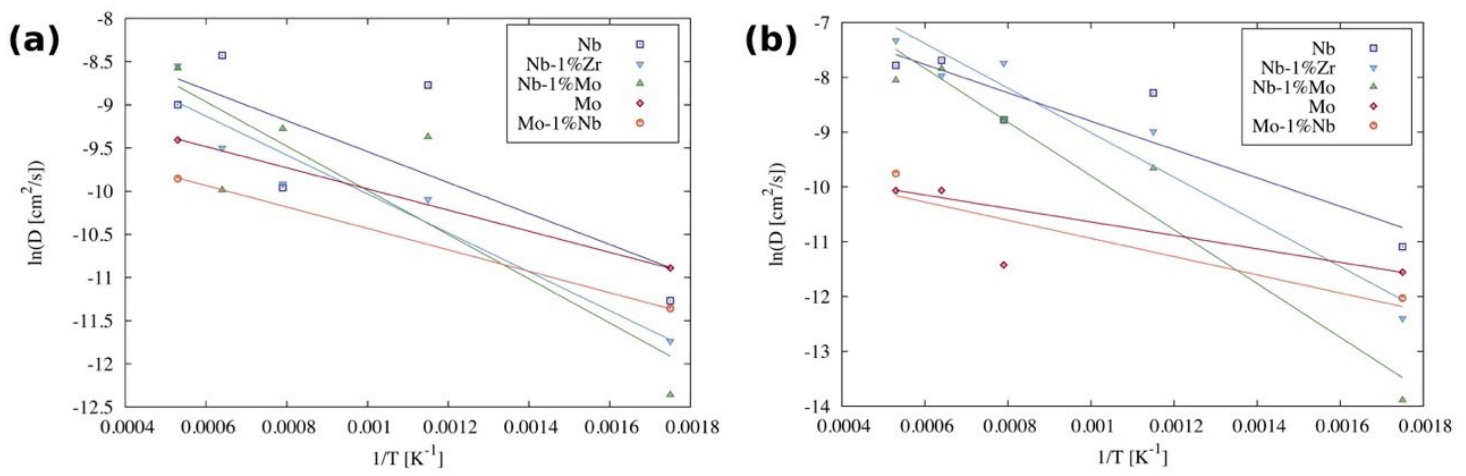

Figure 6.5 Diffusion coefficients for (a) $\mathrm{O}$ and (b) $\mathrm{H}$ after each surface is exposed to $\mathrm{H}_{2} \mathrm{O}$. 
Table 6.2 Diffusion coefficient pre-factors and activation energies of $\mathrm{O}$ and $\mathrm{H}$ for each surface exposed to $\mathrm{H}_{2} \mathrm{O}$.

\begin{tabular}{llllllll}
\hline \multicolumn{2}{l}{$\mathrm{O}$} & & & $\mathrm{H}$ & & \\
& $\mathrm{D}_{0}\left[\mathrm{~cm}^{\wedge} 2 / \mathrm{s}\right]$ & $\mathrm{E}_{\mathrm{A}}[\mathrm{eV}]$ & $\mathrm{E}_{\mathrm{A}}[\mathrm{kJ} / \mathrm{mol}]$ & $\mathrm{D}_{0}\left[\mathrm{~cm}{ }^{\wedge} / \mathrm{s}\right]$ & $\mathrm{E}_{\mathrm{A}}[\mathrm{eV}]$ & $\mathrm{E}_{\mathrm{A}}[\mathrm{kJ} / \mathrm{mol}]$ \\
\hline $\mathrm{Nb}$ & $4.31 \mathrm{E}-04$ & 0.15 & 14.90 & $2.01 \mathrm{E}-03$ & 0.22 & 21.53 \\
$\mathrm{Nb}-1 \% \mathrm{Zr}$ & $4.18 \mathrm{E}-04$ & 0.19 & 18.72 & $7.15 \mathrm{E}-03$ & 0.35 & 33.84 \\
$\mathrm{Nb}-1 \% \mathrm{Mo}$ & $5.93 \mathrm{E}-04$ & 0.22 & 21.28 & $7.52 \mathrm{E}-03$ & 0.42 & 40.81 \\
$\mathrm{Mo}$ & $1.58 \mathrm{E}-04$ & 0.11 & 10.18 & $8.19 \mathrm{E}-05$ & 0.11 & 10.22 \\
$\mathrm{Mo}-1 \% \mathrm{Nb}$ & $1.02 \mathrm{E}-04$ & 0.11 & 10.32 & $9.33 \mathrm{E}-05$ & 0.16 & 15.60 \\
\hline
\end{tabular}

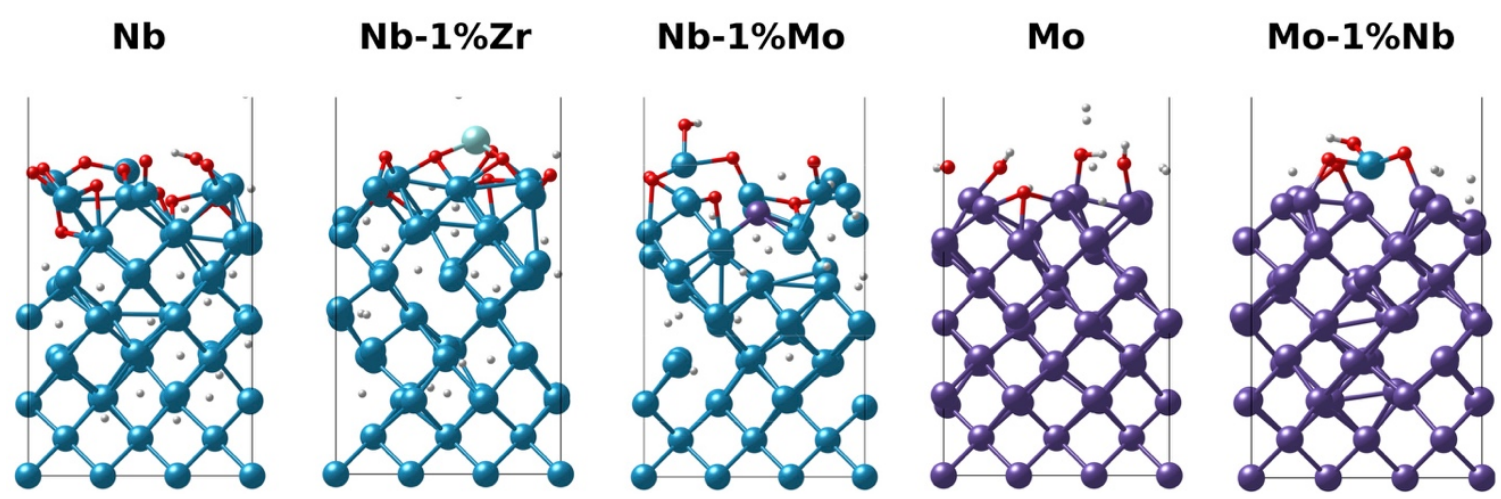

Figure 6.6 Atomic structures of metals under 2 monolayers of $\mathrm{H}_{2} \mathrm{O}$ after 10 ps at $1600{ }^{\circ} \mathrm{C}$. Blue, purple, teal, red, and white atoms represent $\mathrm{Nb}, \mathrm{Mo}, \mathrm{Zr}, \mathrm{O}$, and $\mathrm{H}$ respectively. 
(a)

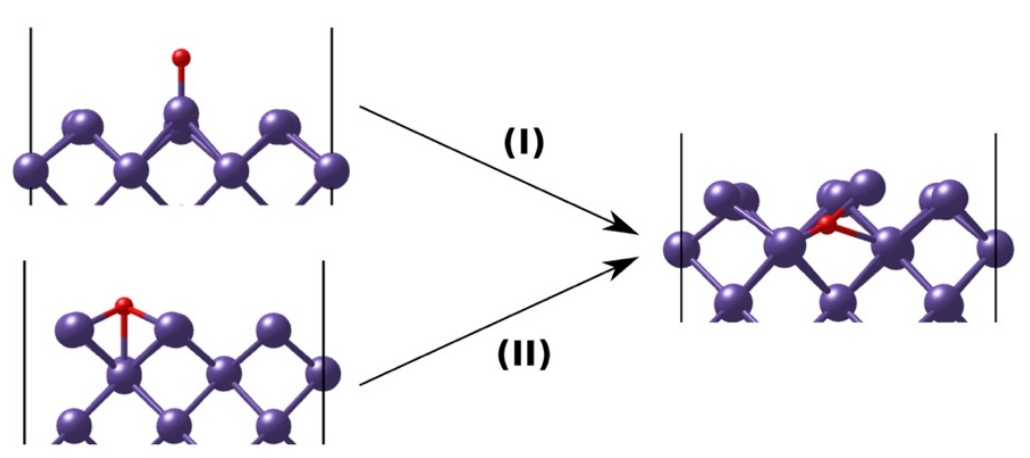

(b)

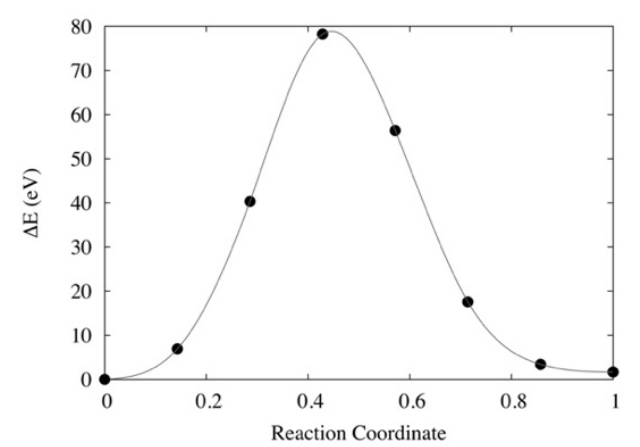

(c)

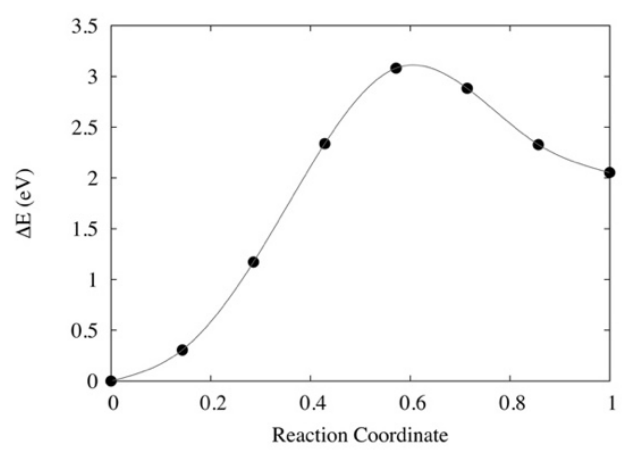

Figure 6.7 Nudged elastic band calculations for $\mathrm{O}$ intercalation into the Mo surface. In reaction pathway (I), $O$ begins at a Mo-top site and transitions to an interstitial site. In reaction pathway (II), $O$ begins at a hollow site and transitions to the interstitial site. The transition energies for pathway (I) are shown in (b) and pathway (II) are shown (c).

The ELFs for the surfaces exposed to $\mathrm{H}_{2} \mathrm{O}$ are shown in Figure 6.8. For the $\mathrm{Nb}$ surface, charge is primarily localized to $\mathrm{H}_{2}$ and the adsorbed $\mathrm{H}$ atoms. At the $\mathrm{Nb}-1 \% \mathrm{Zr}$ surface, charge is localized to the adsorbed $\mathrm{H}$ and adsorbed $\mathrm{O}$. At the $\mathrm{Nb}-1 \% \mathrm{Mo}$ surface, charge remains localized to the $\mathrm{H}_{2} \mathrm{O}$. No charge is localized to Mo and very little is localized to the adsorbed $\mathrm{H}$. At the Mo surface, charge remains localized to the $\mathrm{H}_{2} \mathrm{O}$ and $\mathrm{OH}$. These adsorbates only weakly interact with the surface, likely due to Van der Waals forces. For $\mathrm{Mo}-1 \% \mathrm{Nb}$, charge is localized to the $\mathrm{Nb}$ and the $\mathrm{O}$ adsorbed to the $\mathrm{Nb}$. 
Nb

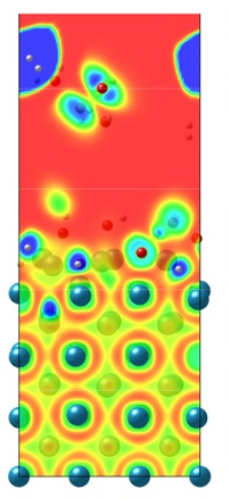

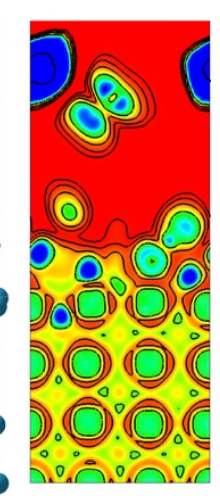

Nb-1\%Zr
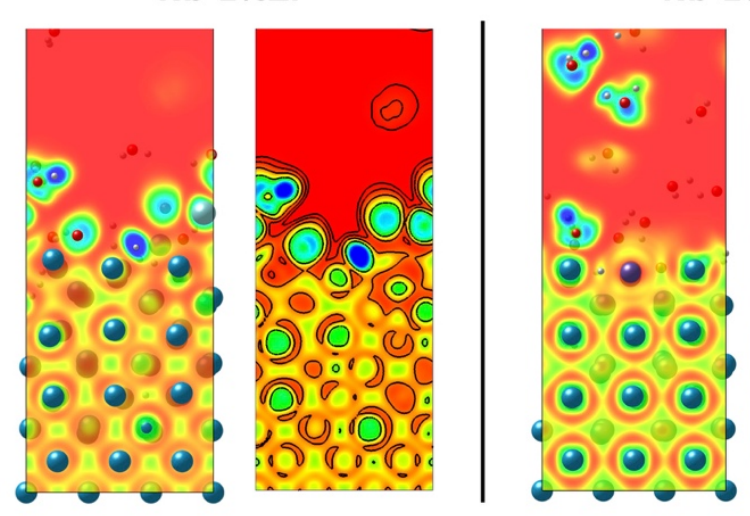

Nb-1\%Mo

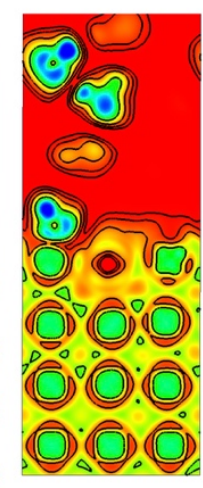

Mo
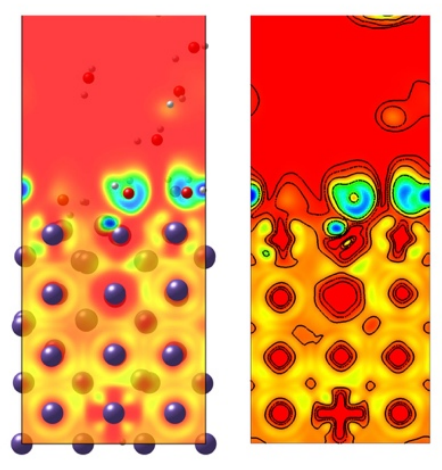

Mo-1\%Nb
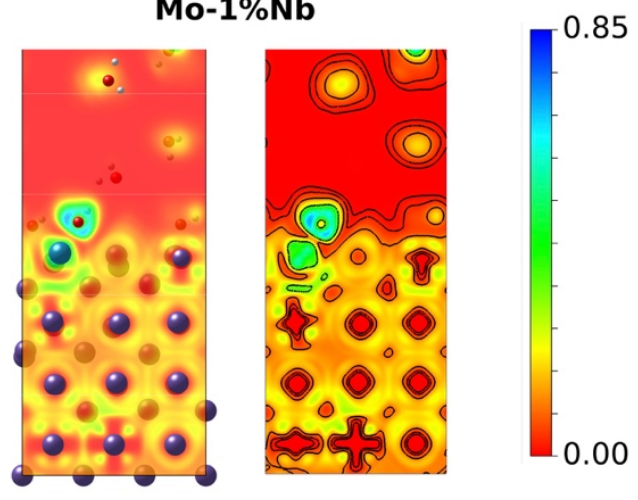

Figure 6.8 Electron Localization Functions of metal surfaces under $\mathrm{H}_{2} \mathrm{O}$ after 1 ps at $1600{ }^{\circ} \mathrm{C}$. Electrons are resolved from $-2 \mathrm{eV}$ to the Fermi level. Contour lines indicate areas with the same energy and are consistent across structures. Blue and red indicate high and low probability of finding a localized electron, respectively.

\subsection{Discussion}

After examining the diffusion coefficient trendlines, $\mathrm{Nb}-1 \% \mathrm{Zr}$ is predicted to resist oxidation better than $\mathrm{Nb}$ at all temperatures. Of the predominantly $\mathrm{Nb}$ alloys, $\mathrm{Nb}-$ $1 \% \mathrm{Zr}$ is the most resistant to oxidation from 20 to approximately $600{ }^{\circ} \mathrm{C}$. Above $600{ }^{\circ} \mathrm{C}$, $\mathrm{Nb}-1 \% \mathrm{Mo}$ is expected to be the most resistant to oxidation of the $\mathrm{Nb}$ alloys. While $\mathrm{Nb}-$ $1 \%$ Mo has the lowest activation energy to oxidation, this is offset by its low diffusion coefficient pre-factor. $\mathrm{Nb}-1 \% \mathrm{Zr}$ and $\mathrm{Nb}-1 \% \mathrm{Mo}$ demonstrate two different strategies for mitigating oxidation. In $\mathrm{Nb}-1 \% \mathrm{Zr}$, $\mathrm{O}$ atoms preferentially bond with $\mathrm{Zr}$ over $\mathrm{Nb}$. In $\mathrm{Nb}-$ $1 \% \mathrm{Mo}, \mathrm{O}$ prefers to not bond with Mo, which creates a local region resistant to bonding 
as the $\mathrm{O}$ seeks $\mathrm{Nb}$ rich regions to adsorb to. The Mo alloys generally have lower $\mathrm{O}$ diffusion coefficients than the $\mathrm{Nb}$ alloys, with $\mathrm{Mo}-1 \% \mathrm{Nb}$ having the best overall oxidation resistance. In the Mo alloys, after $\mathrm{O}$ adsorption charge appears to relocate to the Mo bulk layers rather than alloy the $\mathrm{O}$ to gain electrons.

When exposed to $\mathrm{H} 2 \mathrm{O}, \mathrm{Nb}$ has the worst overall performance. $\mathrm{Nb}-1 \% \mathrm{Zr}$ and $\mathrm{Nb}-$ 1\%Mo perform comparably with regards to $\mathrm{O}$ diffusion due to $\mathrm{H} 2 \mathrm{O}$ exposure, though $\mathrm{Nb}-1 \%$ Mo outperforms $\mathrm{Nb}-1 \% \mathrm{Zr}$ with regards to $\mathrm{H}$ uptake. $\mathrm{Mo}-1 \% \mathrm{Nb}$ is the most resistant to both $\mathrm{O}$ and $\mathrm{H}$ diffusion from $\mathrm{H} 2 \mathrm{O}$ exposure. In order for corrosion to occur, surface adsorption must be more favorable to the $\mathrm{H}$ and $\mathrm{O}$ atoms than remaining in molecular form. Mo surfaces have such little valence electron localization where adsorption is not favorable. Additionally, in the case of $\mathrm{Mo}-1 \% \mathrm{Nb}$, the O preferentially bonds with $\mathrm{Nb}$ over Mo.

In experimental studies of $\mathrm{Nb}$ oxidation, the diffusion coefficient pre-factor has ranged from $2.72 \times 103$ to $1.70 \times 102 \mathrm{~cm} 2 / \mathrm{s}$ and the activation energy from 85.4 to 108 $\mathrm{kJ} / \mathrm{mol}$. This work predicts a $\mathrm{Nb}$ oxidation pre-factor an order of magnitude lower and much lower activation energy. These variations may be expected from the pristine nature of AIMD simulations [14] and the high pressures utilized in order to examine oxidation mechanisms on the AIMD timescale.

\subsection{Conclusion}

Oxidation and corrosion resistance of pure $\mathrm{Nb}$, pure $\mathrm{Mo}$, and three candidate HTIR materials: $\mathrm{Nb}-1 \% \mathrm{Zr}, \mathrm{Nb}-1 \% \mathrm{Mo}$, and $\mathrm{Mo}-1 \% \mathrm{Nb}$ were investigated using AIMD and DFT. Our simulations suggest Mo- $1 \% \mathrm{Nb}$ would be the most resistant of the studied alloys to both oxidation and corrosion. Of the predominantly $\mathrm{Nb}$ alloys, $\mathrm{Nb}-1 \% \mathrm{Zr}$ is most 
oxidation resistant up to $600{ }^{\circ} \mathrm{C}$ while $\mathrm{Nb}-1 \%$ Mo is most oxidation resistant from 600 to $1600{ }^{\circ} \mathrm{C} . \mathrm{Nb}-1 \% \mathrm{Mo}$ is also expected to have the best overall corrosion resistance of the $\mathrm{Nb}$ alloys.

\section{Acknowledgements:}

The authors would like to thank the Center for Advanced Energy Studies for supporting this effort, providing access, and enabling collaboration that otherwise may have been unavailable. This research made use of the resources of the High Performance Computing Center at Idaho National Laboratory, which is supported by the Office of Nuclear Energy of the U.S. Department of Energy and the Nuclear Science User Facilities under Contract No.DE-AC07-05ID14517. 


\section{References}

[1] B.G. Kim, J.L. Rempe, J. Villard, S. Solstad, Review Paper: Review of Instrumentation for Irradiation Testing of Nuclear Fuels and Materials, Radiat. Meas. Gen. Instrum. 176 (2011) 155-187. doi:10.13182/NT11-A13294.

[2] S.J. Zinkle, G.S. Was, Materials challenges in nuclear energy, Acta Mater. 61 (2013) 735-758. doi:10.1016/j.actamat.2012.11.004.

[3] K.L. Murty, I. Charit, An introduction to nuclear materials : fundamentals and applications, Wiley-VCH, 2013.

[4] G.S. Was, D. Petti, S. Ukai, S. Zinkle, Materials for future nuclear energy systems, J. Nucl. Mater. 527 (2019) 1-16. doi:10.1016/j.jnucmat.2019.151837.

[5] S.J. Zinkle, K.A. Terrani, J.C. Gehin, L.J. Ott, L.L. Snead, Accident tolerant fuels for LWRs: A perspective, J. Nucl. Mater. 448 (2014) 374-379. doi:10.1016/j.jnucmat.2013.12.005.

[6] J.L. Rempe, D.L. Knudson, K.G. Condie, S.C. Wilkins, Thermocouples for HighTemperature In-Pile Testing, Nucl. Technol. 156 (2006) 320-331. doi:10.13182/NT06-A3794.

[7] A.J. Palmer, R.S. Skifton, M. Scervini, D.C. Haggard, W.D. Swank, Performance of Custom-Made Very High Temperature Thermocouples in the Advanced Gas Reactor Experiment AGR-5/6/7 during Irradiation in the Advanced Test Reactor, EPJ Web Conf. ANIMMA 2019. 225 (2020). doi:10.1051/epjconf/202022504010.

[8] J.L. Rempe, D.L. Knudson, K.G. Condie, J.C. Crepeau, J.E. Daw, S.C. Wilkins, Options Extending the Applicability of High-Temperature Irradiation-Resistant Thermocouples, Nucl. Technol. 167 (2009) 169-177. doi:10.13182/NT09-14.

[9] J. Daw, J. Crepeau, J. Rempe, D. Knudson, K. Condie, C. Wilkins, Initial Results from Investigations to Enhance the Performance of High Temperature IrradiationResistant Thermocouples, J. Power Energy Syst. 2 (2008) 854-863.

doi:10.1299/jpes.2.854. 
[10] R.A. Perkins, R.A. Padgett Jr., Oxygen Diffusion in Niobium and Nb-Zr Alloys, Acta Metall. 25 (1977) 1221-1230.

[11] R. Kirchheim, Metals as Sinks and Barriers for Interstitial Diffusion with Examples for Oxygen Diffusion in Copper, Niobium and Tantalum, Acta Metall. 27 (1978) 869-878.

[12] J.T. Clenny, C.J. Rosa, Nitridation kinetics of niobium in the temperature range of 873 to 1273 K, Metall. Trans. A. 11 (1980) 1385-1389. doi:10.1007/BF02654521.

[13] E.A. Gulbransen, K.F. Andrew, F.A. Brassart, Oxidation of Molybdenum $550^{\circ}$ to $1700^{\circ} \mathrm{C}$, J. Electrochem. Soc. 110 (1963) 952-959. doi:10.1149/1.2425918.

[14] B. Beeler, D. Andersson, C. Jiang, Y. Zhang, Ab initio molecular dynamics investigation of point defects in $\gamma$-U, J. Nucl. Mater. 545 (2021). doi:10.1016/j.jnucmat.2020.152714.

[15] Y.-J. Zhang, J.-H. Lan, C.-Z. Wang, Q.-Y. Wu, T. Bo, Z.-F. Chai, W.-Q. Shi, Theoretical Investigation on Incorporation and Diffusion Properties of Xe in Uranium Mononitride, J. Phys. Chem. C. 119 (2015) 5783-5789. doi:10.1021/jp510219a.

[16] J. Xi, H. Jiang, C. Liu, D. Morgan, I. Szlufarska, Corrosion of Si, C, and SiC in molten salt, Corros. Sci. 146 (2019) 1-9. doi:10.1016/j.corsci.2018.10.027.

[17] J. Xi, C. Liu, D. Morgan, I. Szlufarska, An Unexpected Role of H During SiC Corrosion in Water, J. Phys. Chem. C. 124 (2020) 9400.

doi:10.1021/acs.jpcc.0c02027.

[18] G. Kresse, J. Furthmüller, Efficient iterative schemes for ab initio total-energy calculations using a plane-wave basis set, Phys. Rev. B. 54 (1996) 11169-11186. doi:10.1103/PhysRevB.54.11169.

[19] J.P. Perdew, K. Burke, M. Ernzerhof, Generalized Gradient Approximation Made Simple, 77 (1996) 3865-3868. doi:doi.org/10.1103/PhysRevLett.77.3865. 
[20] B. Silvi, A. Savin, Classification of chemical bonds based on topological analysis of electron localization functions, Lett. to Nat. 371 (1994) 683-686. doi:10.1038/371683a0.

[21] S.L. Dudarev, G.A. Botton, S.Y. Savrasov, Z. Szotek, W.M. Temmerman, A.P. Sutton, Electronic Structure and Elastic Properties of Strongly Correlated Metal Oxides from First Principles: LSDA + U, SIC-LSDA and EELS Study of UO2 and NiO, Phys. Status Solidi. 166 (1998) 429-443. doi:10.1002/(SICI)1521396X(199803)166:1<429::AID-PSSA429>3.0.CO;2-F.

[22] S. Lutfalla, V. Shapovalov, A.T. Bell, Calibration of the DFT/GGA+U Method for Determination of Reduction Energies for Transition and Rare Earth Metal Oxides of Ti, V, Mo, and Ce, J. Chem. Theory Comput. 7 (2011) 2218-2223. doi:10.1021/ct200202g.

[23] H. Kamisaka, T. Suenaga, H. Nakamura, K. Yamashita, DFT-Based Theoretical Calculations of $\mathrm{Nb}$-and W-Doped Anatase TiO 2 : Complex Formation between W Dopants and Oxygen Vacancies, J. Phys. Chem. C. 114 (2010) 12777-12783. doi:10.1021/jp104355q.

[24] G. Henkelman, B.P. Uberuaga, H. Jónsson, A climbing image nudged elastic band method for finding saddle points and minimum energy paths, J. Chem. Phys. 113 (2000) 9901. doi:10.1063/1.1329672.

[25] E.L. Sikorski, T.H. da Silva, L.K. Aagesen, B.J. Jaques, L. Li, First-principles comparative study of UN and Zr corrosion, J. Nucl. Mater. 523 (2019) 402-412. doi:10.1016/j.jnucmat.2019.06.017.

[26] L.-M. Liu, R. Car, A. Selloni, D.M. Dabbs, I.A. Aksay, R.A. Yetter, Enhanced Thermal Decomposition of Nitromethane on Functionalized Graphene Sheets: $\mathrm{Ab}$ Initio Molecular Dynamics Simulations, J. Am. Chem. Soc. 134 (2012) 1901119016. doi:10.1021/ja3058277.

[27] Y. Yang, X. Shen, Y.-F. Han, Diffusion mechanisms of metal atoms in Pd Au bimetallic catalyst under $\mathrm{CO}$ atmosphere based on ab initio molecular dynamics, Appl. Surf. Sci. 483 (2019) 991-1005. doi:10.1016/j.apsusc.2019.04.036. 


\section{CHAPTER SEVEN: CONCLUSIONS}

The goal of this dissertation is to improve the understanding of UN corrosion and HTIR-TC performance using first-principles. UN has the potential to increase fuel economy and safety margins if a material solution to its corrosion is developed. HTIRTCs can provide vital temperature data from research reactors studying advanced nuclear materials. HTIR-TC development seeks to increase temperature resolution and lifetime in-pile. Both systems were studied using first-principles methods which allow for precise control of composition and can investigate atomic and electronic structure mechanisms and properties. DFT calculations were performed that revealed atomic and electronic mechanisms in UN corrosion initiation and determined the most energetically favorable surface magnetic structure. A method was developed and validated that can predict the performance of HTIR-TC thermoelements. Oxidation and corrosion mechanisms at thermoelement surfaces were investigated to predict which may be most resistant to research reactor coolant.

In Chapter 3, UN corrosion was studied to identify atomistic and electronic corrosion initiation mechanisms (Objective 1). Dissociated water was first applied to the $\mathrm{Zr}$ (1000) surface. Since the $\mathrm{Zr}$ corrosion mechanism is better understood, this system was used to validate the ability of DFT methods to reveal corrosion mechanisms. The combination of atomic structure optimization and ELF revealed water dissociation, $\mathrm{O}$ adsorption, a positively charged $\mathrm{Zr}$ surface, $\mathrm{O}$ intercalation, and charge relocation to $\mathrm{H}$, consistent with the $\mathrm{Zr}$ corrosion mechanism. Dissociated water was then applied to UN 
(100) and (110) surfaces. Atomic structure optimization showed that while Zr oxide formation is initiated with $\mathrm{O}$ intercalation, in $\mathrm{UN}$ the oxynitride begins to form by pulling U away from the surface. UN (110), the less stable of the two investigated surfaces, was found to be more susceptible to corrosion. The ELF revealed that, like $\mathrm{Zr}$, UN forms a positively charged surface and charge relocates to H. LDOS and PCD showed valence electrons from $-2 \mathrm{eV}$ to the Fermi energy were primarily localized to $\mathrm{U}$ atoms. PCD also revealed how the bonding between the terminating UN layers and the rest of the bulk began to weaken after interaction with dissociated water. The lack of valence electrons localized to $\mathrm{N}$ atoms helps explain the prevalence of residual unreacted $\mathrm{N}$ after corrosion and why N-rich UN films are more corrosion resistant. With this improved understanding of UN corrosion, future first-principles studies could examine whether candidate additives reduce $U$ displacement or alter the electronic profile of the surface to make corrosion less favorable.

In Chapter 4, UN surface magnetic treatments and their effects on corrosion properties were investigated with consideration of the Hubbard U-term (Objective 2). If properties from DFT are to be used to parameterize larger length-scale studies of UN, the results should be as accurate as possible to ensure accurate physical mechanisms. With no consensus among DFT nor experimental studies of the UN surface magnetic structure, inaccurate corrosion mechanisms may be reached from modeling. The Hubbard U-term has been found to be necessary to model AFM as the most energetically favorable UN bulk structure, in agreement with experiment. To determine the most energetically favorable UN surface magnetic structure with the Hubbard U-term, U-ramps were performed on five magnetic surface treatments. AFM and FM as well as three hybrid 
configurations were studied. The U-ramp was performed up to $2.0 \mathrm{eV}$, including all effective U-terms that have previously been recommended for UN. Unlike bulk UN, FM treatment was found to be the most stable for all effective U-terms. The U-ramp also revealed the type-1 AFM structure transitions to a new structure of spin-up, spin-down, and half spin-up half spin-down (100) planes above $U_{\text {eff }}=1.6 \mathrm{eV}$. To determine the effect of different magnetic treatments on corrosion results, the three magnetic treatments used in the literature: AFM, FM, and NM were applied to the UN surface. An OH was optimized at each surface and bond lengths, bond angles, adsorption energies, LDOS, PCD, and ELF were compared. Bond lengths, bond angles, and adsorption energies varied by up to $0.05 \AA, 17.95^{\circ}$, and $0.82 \mathrm{eV}$, respectively, across the three magnetic structures. The electronic maps showed similar trends across magnetic treatments, though finer resolution of bond weakening was visible in the FM and AFM treatments. This work shows that the FM structure remains the most energetically favorable treatment of the UN surface with consideration of the Hubbard U-term. If an AFM type-1 structure is used, a metastability treatment should be applied to ensure the structure reaches a ground state. Future AIMD studies that use NM treatment can expect to see similar overall trends in atomic optimization and electronic mapping to spin-polarized calculations, though adsorption energies may vary on the order of $0.82 \mathrm{eV}$.

In Chapter 5, a method to predict the Seebeck coefficient, which corresponds to performance, of HTIR thermoelements was developed. To obtain experimental data to validate the method against, special TCs composed of HTIR-Mo paired with Pt and HTIR-Nb paired with Pt were constructed. The voltage of the special TCs was recorded opposite a Type-B TC during a heat ramp to $1600{ }^{\circ} \mathrm{C}$. The Pt contribution to the voltage 
was then subtracted out to obtain absolute emfs for HTIR-Mo and HTIR-Nb. The method began by comparing the Seebeck coefficients that could be obtained from DFT for pure Mo and pure $\mathrm{Nb}$ to experimental values. After DFT was shown to be able to model the pure metals with reasonable agreement to experiment, the La and $\mathrm{P}$ additives were introduced to Mo and $\mathrm{Nb}$ supercells, respectively. However, the predicted voltages of these supercells were found to not agree experiment as temperature increased. In order to include temperature effects on the atomic structures, the supercells were then optimized using AIMD. O was also added to the supercells for the AIMD simulations to better match experimental compositions. The AIMD heat treatment simulated the ramp up to $1600{ }^{\circ} \mathrm{C}$, the heat soak, the return to room temperature, as well as a subsequent ramp to simulate subsequent insertion into a research reactor. After obtaining the voltages for the AIMD structures, the $600{ }^{\circ} \mathrm{C}$ AIMD structures agreed best with experiment. The $600{ }^{\circ} \mathrm{C}$ structures are likely the best representation of the average structure in experiment. RDFs were also plotted for the AIMD structures and demonstrated how the heat treatment introduces the structural changes due to heat treatment so that they can be included in the calibration. O was found to increase the Seebeck coefficient of Mo. La causes a parabolic change, leading to an increased Seebeck coefficient from 20 to $1000{ }^{\circ} \mathrm{C}$ and reduced Seebeck coefficient above $1000{ }^{\circ} \mathrm{C}$. Heat treatment generally increases the Seebeck coefficient of Mo. In Nb, O leads to a decrease in Seebeck coefficient. P leads to an increase, but this is largely counteracted by the decrease due to temperature. The combination of AIMD and BTE was sensitive to compositional changes ranging from $0.13-1.13$ wt.\% and considered temperature effects. This predictive method could be 
used to predict performance changes due to irradiation damage, oxidation, and corrosion as well as identify developmental alloys that may have higher temperature resolution.

In Chapter 6, oxidation and corrosion of 5 HTIR thermoelement materials were studied (Objective 4). Using a coaxial HTIR-TC build, thermoelement materials may be exposed to research reactor coolant. To investigate thermoelement behavior under oxidation and corrosion conditions, pure $\mathrm{Nb}$, pure $\mathrm{Mo}, \mathrm{Nb}-1 \% \mathrm{Zr}, \mathrm{Nb}-1 \% \mathrm{Mo}$, and Mo$1 \% \mathrm{Nb}$ were exposed to 2 monolayers of $\mathrm{O} 2$ and $\mathrm{H} 2 \mathrm{O}$ using AIMD. Each thermoelement and adsorbate system was simulated at $20,300,600,1000,1300$, and $1600{ }^{\circ} \mathrm{C}$ to obtain the diffusion coefficients. After exposure to $\mathrm{O} 2, \mathrm{O}$ was able to intercalate to a greater depth of the predominantly $\mathrm{Nb}$ surfaces than the Mo surfaces. Mo- $1 \% \mathrm{Nb}$ had the lowest O diffusion coefficient pre-factor of all the thermoelement materials. Of the predominantly $\mathrm{Nb}$ materials, $\mathrm{Nb}-1 \% \mathrm{Zr}$ had the highest energy barrier to $\mathrm{O}$ diffusion while $\mathrm{Nb}-1 \% \mathrm{Mo}$ had the lowest diffusion coefficient pre-factor. This resulted in most oxidation resistant alloys being $\mathrm{Nb}-1 \% \mathrm{Zr}$ from 20 to $600{ }^{\circ} \mathrm{C}$ and $\mathrm{Nb}-1 \% \mathrm{Mo}$ from 600 to $1600{ }^{\circ} \mathrm{C}$. The PCDs reveal two different methods of mitigating oxidation. For $\mathrm{Nb}-1 \% \mathrm{Zr}$, O prefers to bond with $\mathrm{Zr}$ over $\mathrm{Nb}$. For $\mathrm{Nb}-1 \% \mathrm{Mo}$, bonding with Mo is unfavorable to the $\mathrm{O}$, which creates an area on the surface that $\mathrm{O}$ will avoid. For the predominantly Mo surfaces, charge from adsorbed $\mathrm{O}$ is redistributed into the bulk layers. After H2O exposure, $\mathrm{Nb}-1 \% \mathrm{Mo}$ and $\mathrm{Nb}-1 \% \mathrm{Zr}$ have similar $\mathrm{O}$ diffusion coefficients, but $\mathrm{Nb}-1 \% \mathrm{Mo}$ is slightly more resistant to $\mathrm{H}$ diffusion. $\mathrm{Mo}-1 \% \mathrm{Nb}$ is generally the most corrosion resistant of the studied alloys, but the activation energies of $\mathrm{O}$ diffusion appear relatively low. This is due to the $\mathrm{O}$ being limited to surface adsorption, and thus the diffusion activation energy gives the value for surface adsorption rather than diffusion into the 
surface. To better determine the activation energy for O diffusion into the surface, NEB calculations were performed revealing a much higher activation energy. In summary, Mo- $1.35 \mathrm{wt} . \% \mathrm{Nb}$ resisted oxidation and corrosion the most of the studied thermoelement materials. If using a predominantly $\mathrm{Nb}$ material as the exposed thermoelement, $\mathrm{Nb}$ 1.36wt.\%Zr is recommended for an air-cooled reactor up to $600{ }^{\circ} \mathrm{C}, \mathrm{Nb}-1.43 \mathrm{wt} . \% \mathrm{Mo}$ for an air-cooled reactor from $600-1000{ }^{\circ} \mathrm{C}$, and $\mathrm{Nb}-1.43 \mathrm{wt} . \% \mathrm{Mo}$ for all temperatures in a water-cooled reactor. It will be interesting to compare these predictions to the longevity of future HTIR-TC builds in-pile.

For UN, this dissertation identified atomic and electronic mechanisms in corrosion initiation. Now, future first-principles studies can investigate whether candidate additives can hinder these mechanisms. The most energetically favorable magnetic structure of the UN surface has been identified so that first-principles studies can be performed at high accuracy. For HTIR-TCs, a method has been developed and validated that can predict HTIR-TC performance. This method can be used to predict performance changes due to material changes and screen candidate additives for optimal temperature resolution. Thermoelement materials have been evaluated in the presence of water and oxidation and recommendations made for which will be the most resistant to reactor coolant. The work of this dissertation as well as the preceding training, projects, and testing required significant computational effort, with over 17 million core hours used on the High Performance Computing Center at INL.

Overall, this work advances the understanding of two advanced nuclear materials. UN work has demonstrated the utility of DFT in studying atomic and electronic reaction mechanisms as well as the effect of magnetism on adsorption properties. While 
multiscale modeling usually begins at first-principles and progresses to larger length scale models, the developed HTIR-TC method demonstrates how robust predictions can be made moving from a larger length scale (AIMD) to a smaller length scale (DFT). The oxidation and corrosion study of thermoelement surfaces reveals different materials strategies that can hinder diffusion. This work contributes to advancing nuclear energy to improve its viability as a power source. More carbon-free power is crucial to reducing emissions so we can mitigate climate change and harm to human life.

Future work should use AIMD or develop potentials to use MD to study UN. Experimental work towards identifying an additive to improve the corrosion resistance of UN could benefit from AIMD/MD studies that could screen additives for stable incorporation into $\mathrm{UN}$. Investigations of mechanisms leading to oxynitride, $\mathrm{U}_{2} \mathrm{~N}_{3}$, and $\mathrm{UO}_{2}$ formation are needed to clarify the UN corrosion mechanism. The HTIR-TC thermoelement prediction method could be used to compare whether a developmental alloy will be less susceptible to drift due to in-pile conditions than either of the current thermoelements. Experimental work capable of isolating the effects of oxidation, corrosion, and transmutation would be interesting to see which hinders HTIR-TC lifetime the most. 